\title{
ANALYSIS OF $D^{0}-\overline{D^{0}}$ MIXING IN $D^{0} \rightarrow K \pi$ DECAYS USING THE CDF II DETECTOR
}

by

\section{NAGESH P. KULKARNI \\ DISSERTATION}

\author{
Submitted to the Graduate School \\ of Wayne State University, \\ Detroit, Michigan
}

in partial fulfillment of the requirements

for the degree of

DOCTOR OF PHILOSOPHY

2009

MAJOR: PHYSICS

Approved By:

Advisor

Date 
(C)COPYRIGHT BY

NAGESH P. KULKARNI

2009

All Rights Reserved 


\section{ACKNOWLEDGEMENTS}

This work would not have been possible without the inspiration, guidance, and help from the many wonderful people from Wayne State University and from Fermilab.

I wish to thank my advisor, Prof. Paul E. Karchin. It was a privilege to work with Prof. Karchin, one of the most experienced people in the field of high energy particle physics. Prof. Karchin provided me excellent mentoring and direction throughout my research work.

My sincere thanks to Prof. Mark Mattson for his guidance on calorimeter electronics and in $D^{0}-\bar{D}^{0}$ mixing analysis. I was fortunate to have Prof. Robert Harr, Prof. Alexey Petrov, Prof. Claude Pruneau, and Prof. John Klein, on my dissertation committee. I would also like to thank Prof. Claude Pruneau for a number of stimulating discussions on Artificial Neural Network. I also had many valuable discussions with Prof. Harr on the $D^{0}-\bar{D}^{0}$ mixing analysis. I express gratitude to all the conveners and the members of the $B$-physics group for their valuable insights.

A special thanks to Mr. Alfredo Gutierrez, for his help in the lab. It was due to him that I was able to implement parallel processing to speed up the analysis.

I received constant encouragement and support from all the faculty members of the Physics Department. It is simply impossible to name everyone who had helped me at WSU. It is also impossible to name everyone who provided me guidance at Fermilab. I would only say here that I had a chance to work with some of the brightest people from all over in the world and I thank you all for providing me with such a great experience.

Finally, I would like to thank my wife, Sindhu, for keeping me motivated throughout these five years. Without her support, I can’t imagine completing my Ph.D. Thanks! 


\section{TABLE OF CONTENTS}

ACKNOWLEDGEMENTS ii

LIST OF TABLES vi

LIST OF FIGURES vi vi

1 CHARM MIXING OVERVIEW 1

1.1 The Standard Model, New Physics, and the Motivation to search for Charm

Mixing . . . . . . . . . . . . . . . . . . . . 1

1.2 Charm Mixing . . . . . . . . . . . . . . . . . . 3

1.3 Measurement of Mixing Parameters . . . . . . . . . . . . . . 6

1.3.1 $D^{0}$ decay to CP-mixed hadronic final states $\ldots \ldots \ldots$

1.3.2 $D^{0}$ decays to $\mathrm{CP}$-even and non-CP eigenstates $\ldots \ldots \ldots$

1.3.3 Semileptonic decays . . . . . . . . . . . . . . . . . . . 7

1.3.4 Time dependent Dalitz plot analysis . . . . . . . . . . . . . 8

1.3.5 Quantum-correlated final states . . . . . . . . . . . . 8

1.4 Experimental Status . . . . . . . . . . . . . . . . . . . 8

2 EXPERIMENTAL SETUP $\quad 14$

2.1 The Tevatron .................................. 14

2.2 The CDF II Detector . . . . . . . . . . . . . . . . . . 16

2.2.1 Silicon Detector System . . . . . . . . . . . . . . . . . . 19

2.2.2 Central Outer Tracker . . . . . . . . . . . . . . . . 20

2.3 Trigger System . . . . . . . . . . . . . . . . . . . . . . 22

2.4 Offline Processing . . . . . . . . . . . . . . . . . . 26

3 ANALYSIS AND RESULTS 27

3.1 The Goal of the Analysis f . . . . . . . . . . . . . . 27

3.2 Event Reconstruction _. . . . . . . . . . . . . . . . . . 28 
3.3 Two Track Trigger $\ldots \ldots \ldots$. . . . . . . . . . . . . . . . 30

3.4 Data Sample . . . . . . . . . . . . . . . . . . . . . . 31

3.5 A Brief History of This Analysis _ . . . . . . . . . . . . . . . 32

3.6 Analysis . . . . . . . . . . . . . . . . . . . . . . . 32

3.6.1 Backgrounds and Basic Cuts . . . . . . . . . . . 32

3.6 .2 The Clean RS Signal . . . . . . . . . . . . . . . . . . . . 38

3.6 .3 The $D^{*}$ Yield Technique $\ldots \ldots \ldots \ldots$

3.6.4 Non-prompt $D^{*}$ Correction . . . . . . . . . . . . . . . . . . 44

3.7 Results . . . . . . . . . . . . . . . . . . . . . . . . 49

$3.7 .1 \mathrm{WS} / \mathrm{RS}$ Ratio . . . . . . . . . . . . . . . 49

3.7 .2 Systematic Uncertainties . . . . . . . . . . . . . . . . . . 50

3.7 .3 Mixing Significance . . . . . . . . . . . . . . . . . 52

3.8 Cross-check of Mixing Significance . . . . . . . . . . . . . . . . . 54

4 SUMMARY AND FUTURE PROSPECTS 58

A ARTIFICIAL NEURAL NETWORK METHOD 60

A.1 Neural network method for $D^{0}-\bar{D}^{0}$ mixing analysis $\ldots \ldots \ldots$. . . . . 60

A.2 Training sample preparation . . . . . . . . . . . . . . . . 62

A.3 Input parameters and settings . . . . . . . . . . . . . . . . . 62

A.4 Some variables used as Neural network inputs . . . . . . . . . . . . . 66

B OTHER ATTEMPTS TO IMPROVE THE ANALYSIS

B.1 $D^{0}$ isolation variable cut $\ldots \ldots \ldots \ldots \ldots$. . . . . . . . . . 74

B.2 Multiple candidate background . . . . . . . . . . . . . . . . 74

C MONTE CARLO SIMULATION STUDIES $\quad 76$

C.1 Monte Carlo Study of Impact Parameter Distribution of $D^{0}$ for Non-Prompt

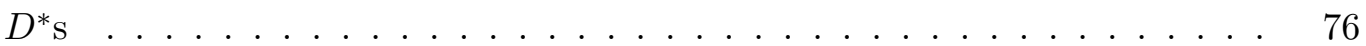

C.2 Lump study . . . . . . . . . . . . . . . . . . . . . . 77

$\begin{array}{lr}\text { REFERENCES } & 84\end{array}$ 


\section{LIST OF TABLES}

1.1 Current values of $D^{0}-\bar{D}^{0}$ mixing parameters provided by BaBar, Belle, and CDF experiments from $D^{0} \rightarrow K \pi$ decays. . . . . . . . . . . . . . 11

3.1 Prompt and Non-Prompt Distribution Fractions _ . . . . . . . . . . 48

3.2 WS/RS ratios for $4.0 \mathrm{fb}^{-1}$ data . . . . . . . . . . . . 50

3.3 The results of the WS/RS ratio fits $\left(4.0 \mathrm{fb}^{-1}\right.$ data $) \ldots \ldots \ldots \ldots$

4.1 The $D^{0}-\bar{D}^{0}$ mixing parameters provided by BaBar and Belle experiments compared with the new CDF result. . . . . . . . . . . . 59

A.1 WS $D^{*}$ significance using the standard method and various neural network cuts. The maximum signal significance is at $\mathrm{NN}$ cut $>0.10 \ldots \ldots 6$ 


\section{LIST OF FIGURES}

1.1 Two $D^{0} \rightarrow K \pi$ decays. A $D^{0}$ decays into $K$ and $\pi$ as Cabibbo favored (a) or as doubly Cabibbo suppressed decay (b). The DCS decay is suppressed by CKM factors at two vertices: $c d W$ vertex and $u s W$ vertex . . . . . . . .

1.2 In long range processes (a), a $D^{0}$ evolves into an intermediate state such as $K^{+} K^{-}$or $\pi^{+} \pi^{-}$and then into $\bar{D}^{0}$ which subsequently decays into $K^{-} \pi^{+}$as Cabibbo favored decay. In short range processes (b) a $\bar{D}^{0}$ is shown decaying into $D^{0}$ through the box diagram. . . . . . . . . . . . . . .

1.3 The BaBar result. The BaBar experiment compares the decay time distributions of $D^{0} \rightarrow K^{+} \pi^{-}$and $D^{0} \rightarrow K^{-} \pi^{+}$. The plot shows combined $D^{0}$ and $\bar{D}^{0} \mathrm{WS}$ candidates with and without mixing fits (with no $\mathrm{CP}$ violation). The residual plot is the difference between the two fits and the structure appearing in that plot corresponds to $D^{0}-\bar{D}^{0} \operatorname{mixing}[19]$. . . . . . . . .

1.4 The Belle results. The plots show simultaneous fits to decay time distributions of the selected (a) $D^{0} \rightarrow K^{+} K^{-}$, (b) $D^{0} \rightarrow K^{-} \pi^{+}$, and (c) $D^{0} \rightarrow \pi^{+} \pi^{-}$ candidates. The fits were performed with $y_{C P}$ as a common free parameter. The shaded areas are the backgrounds. Plot (d) is the ratio of decay time distributions between the $\mathrm{CP}$ even and non-CP decays $[20] . \ldots . . .$.

1.5 Evidence for $D^{0}-\bar{D}^{0}$ mixing. (a) Babar result $\left(384 \mathrm{fb}^{-1}\right)$. The $1-5 \sigma$ contours exclude no-mixing at $3.9 \sigma$ level [19]. (b) $\operatorname{CDF}$ result $\left(1.5 \mathrm{fb}^{-1}\right)$. The Bayesian probability contours equivalent to $1-4 \sigma$. No-mixing point is excluded at $3.8 \sigma$ level $[22] \ldots \ldots \ldots \ldots \ldots$ 
1.6 Visual comparison of Belle (green), BaBar (blue), and CDF (2007) (red) measurements. These are the standard error ellipses based on the fit error matrices of the three results. The BaBar measurements are centered around $x^{\prime 2}=-0.022 \%, y^{\prime}=0.97 \%$. The Belle measurements are centered around $x^{\prime 2}=0.018 \%, y^{\prime}=0.06 \%$. The CDF (2007) measurements are centered around $x^{2}=-0.012 \%, y^{\prime}=0.85 \% \ldots \ldots \ldots \ldots$

1.7 Two dimensional contours for mixing parameters $x$ and $y$ provided by heavy flavor averaging group $[29] . \ldots \ldots \ldots$. . . . . . . . . . . . 13

2.1 Fermilab accelerator chain $[30,31] \ldots \ldots \ldots \ldots$

2.2 A picture of CDF II detector with end plug opened $[31,37] \ldots \ldots \ldots$

2.3 Elevation view of the CDF II detector $[31,37] \ldots \ldots \ldots \ldots$

2.4 Isometric view of the CDF II detector $[31,37] \ldots \ldots \ldots \ldots$

2.5 The $r$ - $z$ view of the CDF II tracking system $[31,37] \ldots \ldots \ldots$

2.6 Components of silicon detector system spanning various pseudo-rapidity $(\eta)$

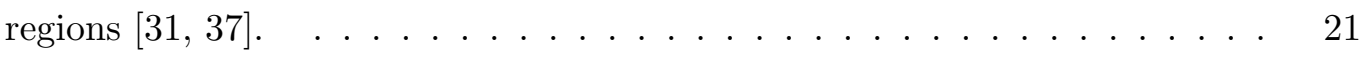

2.7 Silicon Vertex Detector end view $[31,37] \ldots \ldots \ldots \ldots$

2.8 Detection of a particle track in a silicon strip detector $[31,37] . \ldots 22$

2.9 COT superlayers. COT is divided in 8 superlayers, each containing 12 wires, to provide 96 measurement points radially. The number of cells in each superlayer ranges from 168 for SL1 to 480 for SL8, providing a total of 2520 cells $[31,37] \ldots \ldots \ldots \ldots \ldots \ldots \ldots \ldots \ldots \ldots \ldots \ldots \ldots \ldots \ldots \ldots$

2.10 A COT cell. The wire plane makes an angle of $35^{\circ}$ with the radial direction $[31,37] \ldots \ldots \ldots \ldots \ldots \ldots \ldots \ldots \ldots \ldots \ldots \ldots \ldots \ldots \ldots \ldots$

2.11 Block diagram of the three level trigger system at CDF II detector. For the purpose of this analysis, the data from COT and SVX is used. The COT data is processed by XFT (eXtremely Fast Tracker) and XTRP (Extrapolator). The SVX data is processed by Secondary Vertex Tracker (SVT). Level 2 accepts the data from Level $1[31,37] \ldots \ldots \ldots$ 
3.1 Illustration of $D^{*} \rightarrow D^{0} \pi ; D^{0} \rightarrow K \pi$ decay chain at the CDF II detector in the $x$ - $y$ plane transverse to beam line. The $D^{*}$ decays promptly into $\pi$ and $D^{0}$ and the $D^{0}$ further decays into $K$ and $\pi$. The figure also illustrates decay length $\left(L_{x y}\right)$ of $D^{0}$. The impact parameter $d_{0}$ for a kaon track is illustrated

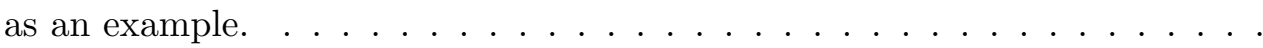

3.2 A sketch to illustrate signal and backgrounds in $K \pi$ mass and mass difference distributions. The $D^{0}(K \pi)$ mass distribution has the range from 1.80 to 1.92 $\mathrm{GeV} / c^{2}$ on the $x$-axis and the number of events per $2 \mathrm{MeV} / c^{2}$ on the $y$-axis. The mass difference distribution has the range from 0 to $30 \mathrm{MeV} / c^{2}$ on the $x$-axis and the number of events per $0.5 \mathrm{MeV} / c^{2}$ on the $y$-axis [44]. . . .

3.3 WS and RS $K \pi$ mass plotted together (using $60 \mathrm{pb}^{-1}$ data). The dashed lines correspond to the world average $D^{0}$ mass, $1.8645 \mathrm{GeV} / c^{2}[45] \ldots$. . .

3.4 WS and RS plot after removing $K K$ and $\pi \pi$ background (using $1.5 \mathrm{fb}^{-1}$ data) [44]

3.5 Opposite assignment mass cut on RS $K \pi$ mass for WS signal using $1.5 \mathrm{fb}^{-1}$

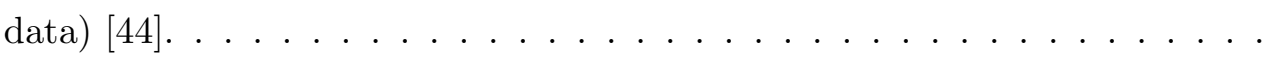

3.6 $\mathrm{dE} / \mathrm{dx}$ ( $Z$ variable) distribution for a kaon track (a) with a kaon hypothesis and (b) with a pion hypothesis. . . . . . . . . . . . 36

$3.7 \mathrm{dE} / \mathrm{dx}$ ( $Z$ variable) distribution for a pion track (a) with a kaon hypothesis and (b) with a pion hypothesis. . . . . . . . . . .

3.8 The right sign and wrong sign distributions with $0.35 \mathrm{fb}^{-1}$ data. The figures on the left are right sign and wrong distributions before the opposite assignment mass cut and PID cuts are applied. The figures on the right are the RS and WS distributions after the application of these cuts. In all these plots, the red colored peak region is the RS(WS) signal. The blue colored broad background is mis-identified background. The green colored linear distribution is from the combinatorial background $[44] . \ldots 38$

3.9 Projection of WS $D^{0}$ fit results obtained from $0.35 \mathrm{fb}^{-1}$ data to illustrate relative amounts of signal and backgrounds $[43] \ldots \ldots \ldots \ldots$ 
3.10 The time integrated RS $D^{0}$ sample. Dark grey is the signal fit, light grey is the background fit. . . . . . . . . . . . . . . . . 40

3.11 RS $K \pi$ mass fit for $2.75<t / \tau<3.00$ and $5.5<\Delta M<6.0 \mathrm{MeV} / c^{2}$. . . . 41

3.12 WS $K \pi$ mass fit for $2.75<t / \tau<3.00$ and $5.5<\Delta M<6.0 \mathrm{MeV} / c^{2}$. . . 42

$3.13 \mathrm{RS} D^{*}$ distribution for $2.75<t / \tau<3.00 \ldots \ldots \ldots \ldots \ldots$

3.14 WS $D^{*}$ distribution for $2.75<t / \tau<3.00$. . . . . . . . . . 43

3.15 Time integrated right $\operatorname{sign} \Delta M$ distribution. $\ldots \ldots \ldots \ldots$

3.16 Time integrated wrong $\operatorname{sign} \Delta M$ distribution. . . . . . . . . . . . . 46

3.17 Impact parameter distribution for RS $D^{0}$ mesons with $5<t / \tau<6$ using 4.0 $\mathrm{fb}^{-1}$ data. . . . . . . . . . . . . . . . . . .

3.18 The ratio of wrong sign to right sign $D^{*}$ s as a function of normalized $D^{0}$ decay time $\left(4.0 \mathrm{fb}^{-1}\right) \ldots \ldots \ldots \ldots \ldots \ldots \ldots$

3.19 RS $K \pi$ mass distribution with quadratic fit function for background (a) without additional Gaussian and (b) with extra Gaussian at $1.83 \mathrm{GeV} / \mathrm{c}^{2}$. The signal shape is a double Gaussian. These plots were made using $1.5 \mathrm{fb}^{-1}$

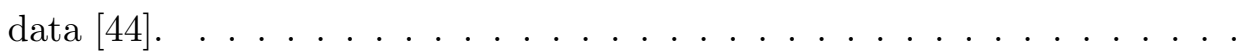

3.20 Bayesian probability contours equivalent to 1 to 6 Gaussian standard deviation. The no-mixing point $\left(x^{\prime 2}=y^{\prime}=0\right)$, shown as a cross, falls on the $5.3 \sigma$ contour. In other words, our result excludes no-mixing hypothesis with 5.3 Gaussian standard deviation. The solid dot shows the measured values of $x^{2}$ and $y^{\prime}$ from the best fit. . . . . . . . . . . . . .

3.21 The simulated experiment and $\delta \chi^{2}$ distribution: the top plot shows a single simulation of a WS/RS ratio. The bottom plot shows the $\delta \chi^{2}$ distribution from 1000 simulated experiments. . . . . . . . . . . . . . . .

3.22 Bayesian contours from a simulated experiment. The no-mixing point is on the $5.0 \sigma$ contour. The solid dot shows the measured values of $x^{\prime 2}$ and $y^{\prime}$ from the best fit. The diamond shows the highest probability point in physically allowed $x^{\prime 2} \ldots \ldots \ldots \ldots \ldots \ldots \ldots \ldots$ 
4.1 Visual comparison of the Belle, BaBar, and the new CDF II results. The plot shows standard error ellipses obtained from the fit error matrices. The BaBar and Belle measurements are the same as in Figure 1.6. The new CDF II measurement is centered around $x^{\prime 2}=0.011 \%$ and $y^{\prime}=0.35 \% . \ldots$.

A.1 A typical artificial neural network has input nodes, synapses, middle layer with hidden nodes, and output node. In the above sketch, the nodes are shown by circles while the arrows are synapses. The network finds a discrimination function by adjusting weights on each of the synapses. . . . . . . .

A.2 The RS and WS $K \pi$ mass in $\mathrm{GeV} / c^{2}$ plotted together to illustrate signal and background samples used for neural network training. The red boxes show the region from where background sample is extracted. The central green area shows the signal region. . . . . . . . . . . . . . .

A.3 Neural network training output. Signal is shown in red color and background in black color. Signal gradually increases and background decreases with NN output. Maximum signal significance is found at NN cut $>0.10$. . . . .

A.4 Comparison of the ratio plots obtained from the neural network method (top) and from the standard analysis (bottom) for $2.5 \mathrm{fb}^{-1}$ data. . . . . . . . .

A.5 Comparison of the transverse momentum of the kaon for signal and background samples. . . . . . . . . . . . . . . .

A.6 Comparison of the transverse momentum of the pion for signal and background samples. . . . . . . . . . . . . . . .

A.7 Comparison of the transverse momentum of the soft pion for signal and back-

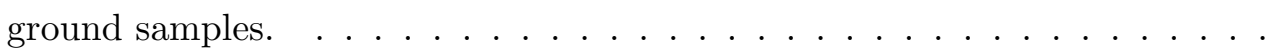

A.8 Comparison of the $\mathrm{Z} 0$ variable-the point of closest approach along the beam line -for the stable pion for signal and background samples. . . . . . . . .

A.9 Comparison of the $\mathrm{Z} 0$ variable-the point of closest approach along the beam line -for the kaon for signal and background samples. . . . . . . . . . . 70

A.10 Comparison of the $\mathrm{Z} 0$ variable-the point of closest approach along the beam line - for the soft pion for signal and background samples. . . . . . . . . 
A.11 Comparison of the impact parameter of the soft pion for signal and background samples. . . . . . . . . . . . . . . . . . . . 71

A.12 Comparison of the $\eta$ variable of the $D^{0}$ for signal and background samples. $\quad 71$

A.13 Comparison of the $\eta$ variable of the soft pion for signal and background samples. 72

A.14 Comparison of the transverse decay length significance of the $D^{0}$ for signal and background samples. . . . . . . . . . . . . . . 72

A.15 Comparison of the $z$ component of the decay length significance of the $D^{0}$ for signal and background samples. . . . . . . . . . . . . . . . . 73

B.1 WS $D^{*}$ yield vs. Isolation of $D^{0}$ cut. The yield is almost flat. It appears that the $D^{0}$ isolation variable is not useful as a standard cut. . . . . . . . . 75

C.1 IP distribution for simulated $B$ to charm decays. Time bin 1 (1.00 to 2.75

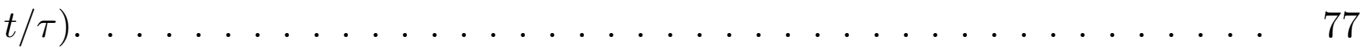

C.2 IP distribution for simulated $B$ to charm decays. Time bin 2 (2.75 to 3.00

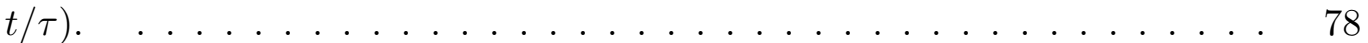

C.3 IP distribution for simulated $B$ to charm decays. Time bin $3(3.00$ to $3.5 t / \tau)$. 78

C.4 IP distribution for simulated $B$ to charm decays. Time bin $4(3.5$ to $4.0 t / \tau)$. 79

C.5 IP distribution for simulated $B$ to charm decays. Time bin 5 (4.00 to $5.5 t / \tau)$. 79

C.6 IP distribution for simulated $B$ to charm decays. Time bin $6(5.5$ to $7.0 t / \tau)$. 80

C.7 IP distribution for simulated $B$ to charm decays. Time bin 7 (7.00 to 8.00

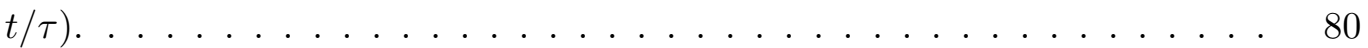

C.8 IP distribution for simulated $B$ to charm decays. Time bin 8 (8.00 to 10.00

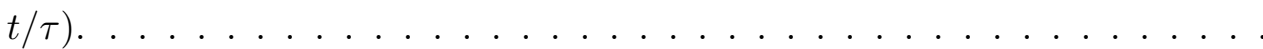

C.9 The $D^{0}$ mass distribution for the $D^{0}$ reconstructed from simulated $D^{0} \rightarrow$ $K^{-} \mu^{+} \nu_{\mu}$ decay assuming the muon as a pion. We applied the standard mass difference cut. The mean invariant mass falls below $1.83 \mathrm{GeV} / c^{2}$. The lump in RS K]pi mass plot was found at $1.83 \mathrm{GeV} / c^{2}$. . . . . . . . . . . . . . . 82

C.10 Invariant mass of $\Lambda_{c}^{+}$reconstructed from simulated $\Lambda_{c}^{+} \rightarrow p^{+} K^{-} \pi^{+}$decay. This was another decay that we thought would cause the lump in RS plot. But the mean invariant mass of $\Lambda_{c}^{+}$found to be well below $1.83 \mathrm{GeV} / c^{2}$. . 


\section{CHAPTER 1}

\section{CHARM MIXING OVERVIEW}

"The universe is not what it used to be, nor what it appears to be .... nor is space what it appears to be. What appears to our eyes as empty space is revealed to our minds a complex medium full of spontaneous activity."

-Frank Wilczek, The Lightness of Being (2008).

Since the discovery of the $D^{0}$ meson, scientists have been searching for $D^{0}-\bar{D}^{0}$ mixing. This chapter describes the formalism of $D^{0}-\bar{D}^{0}$ mixing and the current experimental status.

\subsection{The Standard Model, New Physics, and the Motivation to search for Charm Mixing}

The electroweak (EWK) theory together with quantum chromodynamics (QCD) forms the Standard Model (SM). Built on the concepts of quantum mechanics, relativity, and SU(3) $\times \mathrm{SU}(2) \times \mathrm{U}(1)$ gauge symmetry, the Standard Model summarizes completely the present state of our knowledge of particle physics. Two types of elementary particles are described in the Standard Model: fermions and bosons. Fermions are matter particles of two types: leptons and quarks. The leptons include electron $\left(e^{-}\right)$, muon $\left(\mu^{-}\right)$, and tau $\left(\tau^{-}\right)$particles and the associated neutrinos $\left(\nu_{e}, \nu_{\mu}\right.$, and $\left.\nu_{\tau}\right)$. The quarks include, up $(u)$, down $(d)$, charm $(c)$, strange $(s)$, top $(t)$, and bottom $(b)$. Bosons include twelve force carriers: photon, the $W^{+}, W^{-}$, and $Z^{0}$ bosons, the eight gluons, and Higgs bosons. In addition, every particle has its own anti-particle [1].

It is fascinating that with only few particles and concepts, the Standard Model elegantly describes all the known particle interactions (except gravity).

However, most physicists believe that the Standard Model is not the complete theory of physics; rather, it is only a step towards the formulation of the final theory of physics. One of the shortcomings of the Standard Model is that it has a large number of parameters that must be put into the model by hand. For example, the Standard Model fails in explaining 
the origin of mass. For instance, the mass of the electron cannot be computed within the Standard Model framework. Secondly, the Standard Model leaves out gravity. There are also unanswered questions about dark matter and dark energy.

Many theories and models have been proposed to overcome these shortcomings of the Standard Model, or to answer the outstanding questions. Some of the popular theories are: Super-symmetry, Extra Dimensions, and String Theory. Supersymmetry is the symmetry between fermions and bosons. This is an extension of the Standard Model that predicts a supersymmetric partner for every Standard Model particle. The theory of extra dimensions explores the possibility that the world actually has more than four dimensions. String theory, put in an oversimplified manner, suggests that extremely small vibrating strings are the fundamental building blocks of the universe. The predictions of such theories are usually labelled as "New Physics" or "physics beyond the Standard Model" [3, 4].

There are several ways to probe into new physics in high energy physics experiments. One of them is the "direct search" for the non-SM particles or particle interactions predicted by new theories. For instance, the search for super symmetric partners of SM particles such as squarks or the search for fourth generation quark flavor, would fall in the category of "direct search". Another way to look beyond the Standard Model is to search for any deviations from the Standard Model predictions in the current experimental set-ups. These are the "indirect probes" into new physics. These include the search for some strongly suppressed particle interactions in the Standard Model.

This second type of reasoning; i.e. indirect probes, is the motivation behind the search for mixing and for $\mathrm{CP}$ violation in neutral $D$ meson decays. Neutral $D$ mesons are bound states of charm and anti-up quarks. The charm mixing provides excellent opportunities to constrain the parameter space of a wide range of new physics models through the precise measurement of the mixing parameters of neutral $D$ mesons [5].

Also, $\mathrm{CP}$ violation in charm mixing is predicted to be extremely small in the Standard Model since mixing and relevant decays can be described by physics of the first two quark generations $[10,12]$. So the observation of $\mathrm{CP}$ violation in charm mixing in the current round of experiments will be an unambiguous signal of new physics.

The first evidence of charm mixing was found very recently in 2007. The mixing is now 
fairly established by combining all the published results; however, it was not observed in a single decay channel with more than $5 \sigma$ significance.

This thesis presents the observation of the neutral $D$ meson mixing in $D^{0} \rightarrow K \pi$ channel using the Collider Detector at Fermilab (CDF II). The CDF II experiment is described in the second chapter and the analysis and results are discussed in the third chapter.

\subsection{Charm Mixing}

Mixing is the quantum mechanical phenomena of oscillations between particles and antiparticles. It has been observed in the $K^{0}-\bar{K}^{0}$ and $B^{0}-\bar{B}^{0}$ systems. Evidence for mixing in the $D^{0}-\bar{D}^{0}$ system has been recently found. In analogy with $K^{0}-\bar{K}^{0}$ and $B^{0}-\bar{B}^{0}$ systems, the $D^{0}-\bar{D}^{0}$ system can be described by the Schrodinger equation

$$
i \frac{\partial}{\partial t}\left(\begin{array}{c}
D^{0}(t) \\
\bar{D}^{0}(t)
\end{array}\right)=\left(\mathbf{M}-\frac{i}{2} \boldsymbol{\Gamma}\right)\left(\begin{array}{c}
D^{0}(t) \\
\bar{D}^{0}(t)
\end{array}\right)
$$

where $\mathbf{M}$ and $\boldsymbol{\Gamma}$ are Hermitian matrices. The $\boldsymbol{\Gamma}$ term ensures the particles decay. The chargeparity-time (CPT) invariance requires $M_{11}=M_{22}$ and $\Gamma_{11}=\Gamma_{22}$. In case of negligible $\mathrm{CP}$ violation, the flavor eigenstates $D^{0}$ and $\bar{D}^{0}$ can be written as linear combinations of mass eigenstates $\left|D_{1}\right\rangle$ and $\left|D_{2}\right\rangle$ as

$$
D^{0}=\frac{1}{\sqrt{2}}\left(\left|D_{1}\right\rangle+\left|D_{2}\right\rangle\right), \quad \bar{D}^{0}=\frac{1}{\sqrt{2}}\left(\left|D_{1}\right\rangle-\left|D_{2}\right\rangle\right)
$$

The time evolution of the $D^{0}-\bar{D}^{0}$ system shows the oscillations of $D^{0}$ into $\bar{D}^{0}$ and vice-versa. The mixing is characterized by the difference in masses and decay widths of $\left|D_{1}\right\rangle$ and $\left|D_{2}\right\rangle$ states. The mixing parameters are then defined as

$$
x=\frac{m_{1}-m_{2}}{\Gamma}=\frac{\delta m}{\Gamma} \text { and } y=\frac{\Gamma_{1}-\Gamma_{2}}{2 \Gamma}=\frac{\delta \Gamma}{2 \Gamma},
$$

where $\delta m$ is the mass difference between the mass eigenstates and $\delta \Gamma$ is the decay width difference, with $\Gamma$ being the average decay width of the eigenstates.

The $D$ mesons decay through weak interactions in which charged-current $W^{ \pm}$inter- 
actions couple to physical quarks with the couplings given by the Cabibbo-KobaysahiMaskawa (CKM) matrix. The CKM matrix is a $3 \times 3$ unitary matrix defined as

$$
V_{C K M}=\left(\begin{array}{ccc}
V_{u d} & V_{u s} & V_{u b} \\
V_{c d} & V_{c s} & V_{c b} \\
V_{t d} & V_{t s} & V_{t b}
\end{array}\right)
$$

The magnitudes of CKM elements are determined empirically [13]. The CKM matrix can be written in terms of three mixing angles $\left(\theta_{i j}, i, j\right.$ representing quark generations) and a CP-violating phase $(\delta)$ as:

$$
V_{C K M}=\left(\begin{array}{ccc}
c_{12} c_{13} & s_{12} c_{13} & s_{13} e^{-i \delta} \\
-s_{12} c_{23}-c_{12} s_{23} s_{13} e^{i \delta} & c_{12} c_{23}-s_{12} s_{23} s_{13} e^{i \delta} & s_{23} c_{13} \\
s_{12} s_{23}-c_{12} c_{23} s_{13} e^{i \delta} & -c_{12} s_{23}-s_{12} c_{23} s_{13} e^{i \delta} & c_{23} c_{13}
\end{array}\right)
$$

where $s_{i j}=\sin \theta_{i j}, c_{i j}=\cos \theta_{i j}$ [13]. The Wolfenstein parametrization [17] expands the CKM matrix in terms of $\lambda, A, \rho, \eta$ as:

$$
V_{C K M}=\left(\begin{array}{ccc}
1-\frac{1}{2} \lambda^{2}-\frac{1}{8} \lambda^{3} & \lambda & A \lambda^{3}(\rho-i \eta) \\
-\lambda+\frac{1}{2} A^{2} \lambda^{5}[1-2(\rho+i \eta)] & 1-\frac{1}{2} \lambda^{2}-\frac{1}{8} \lambda^{4}\left(1+4 A^{2}\right) & A \lambda^{2} \\
A \lambda^{3}\left[1-\left(1-\frac{1}{2} \lambda^{2}\right)(\rho+i \eta)\right] & -A \lambda^{2}+\frac{1}{2} A \lambda^{4}[1-2(\rho+i \eta)] & 1-\frac{1}{2} A^{2} \lambda^{4}
\end{array}\right)
$$

where $\lambda \approx 0.23, A \approx 0.81, \rho \approx 0.14$, and $\eta \approx 0.35$. The three matrices are related as follows:

$$
s_{12}=\lambda=\frac{\left|V_{u s}\right|}{\sqrt{\left|V_{u d}\right|^{2}+\left|V_{u s}\right|^{2}}}, \quad s_{23}=A \lambda^{2}=\lambda\left|\frac{V_{c b}}{V_{u s}}\right|, \quad s_{13} e^{i \delta}=V_{u b}^{*}=A \lambda^{3}(\rho+i \eta) .
$$

Weak interactions give rise to hadronic and semileptonic decays. One of the hadronic decay modes has the end products of a kaon and a pion. In semileptonic processes, the final decay products are neutrinos and other leptons along with hadrons. The mixing parameters $x$ and $y$ can be measured in any of these decay modes. This analysis is concerned with the hadronic decay $D^{0} \rightarrow K \pi$. 
The $D^{0} \rightarrow K \pi$ decay can proceed in two ways as shown in the Feynman diagrams in Figure 1.1. The $D^{0} \rightarrow K^{-} \pi^{+}$decay, as shown in Figure 1.1 (a), is called Cabibbo favored (CF) decay and the $D^{0} \rightarrow K^{+} \pi^{-}$decay, as shown in Figure 1.1 (b), is called doubly Cabibbo suppressed (DCS) decay. The CF and DCS decays are also called the "right sign (RS)" and "wrong sign (WS)" decays respectively. The DCS decay is suppressed by CKM factors at two vertices, the $c d W$ vertex and $u s W$ vertex (so the decay is called doubly Cabibbo suppressed decay). The CF decay involves the CKM elements $V_{c s}$ and $V_{u d}$. From Equation 1.1 , the values of these elements are $\approx 1$. The DCS decay involves the CKM elements $V_{c d}$ and $V_{u s}$ and from Equation 1.1, their values are of order $\lambda$. So the probability of occurrence of DCS decay relative to CF decay is of order $\lambda^{4}$ which is approximately 0.003. In fact the measured branching ratio of DCS to CF decay is $\Gamma\left(K^{+} \pi^{-}\right) / \Gamma\left(K^{-} \pi^{+}\right)=$ $3.80 \pm 0.18 \times 10^{-3}[2]$.

(a)
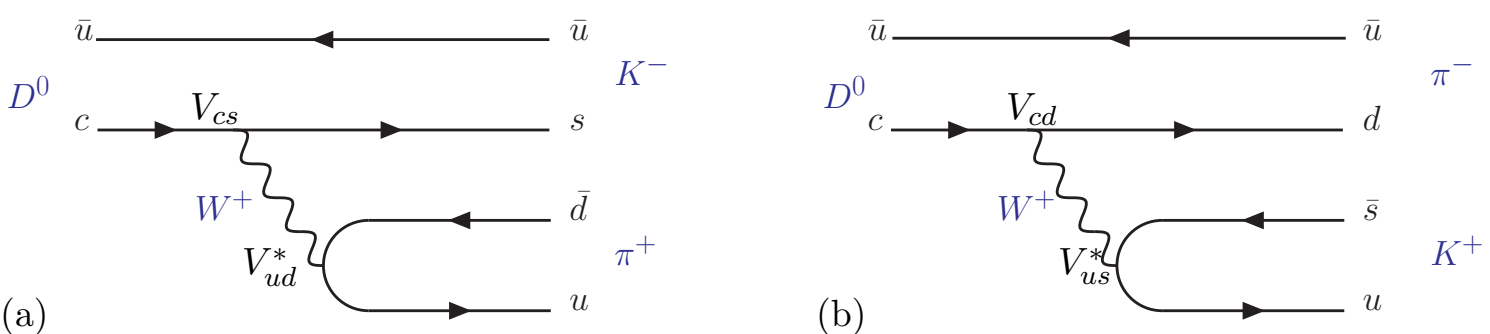

Figure 1.1. Two $D^{0} \rightarrow K \pi$ decays. A $D^{0}$ decays into $K$ and $\pi$ as Cabibbo favored (a) or as doubly Cabibbo suppressed decay (b). The DCS decay is suppressed by CKM factors at two vertices: $c d W$ vertex and $u s W$ vertex

In the Standard Model, charm mixing in the $D^{0} \rightarrow K \pi$ channel can occur through long range processes or through short range processes. In the long range processes, as depicted in Figure $1.2(\mathrm{a})$, a $D^{0}$ evolves into an intermediate state such as $K^{+} K^{-}$or $\pi^{+} \pi^{-}$and then into $\bar{D}^{0}$ which subsequently decays into $K^{-} \pi^{+}$as Cabibbo favored decay.

Figure 1.2 (b) illustrates mixing through short range processes in which a $\bar{D}^{0}$ decay proceeds through box diagram involving $d, s$, and $b$ quarks and advances into $D^{0}$. Since the short-range processes in $D^{0}-\bar{D}^{0}$ system are dominated by light quarks, their contribution is negligible in the Standard Model. The predicted values of the mixing parameters are $x \approx y \approx \mathcal{O}\left(10^{-7}\right)[6]$. This is in contrast with $K^{0}-\bar{K}^{0}$ and $B^{0}-\bar{B}^{0}$ systems in which the box diagrams are dominated by super heavy top quarks. However, exotic particles from 


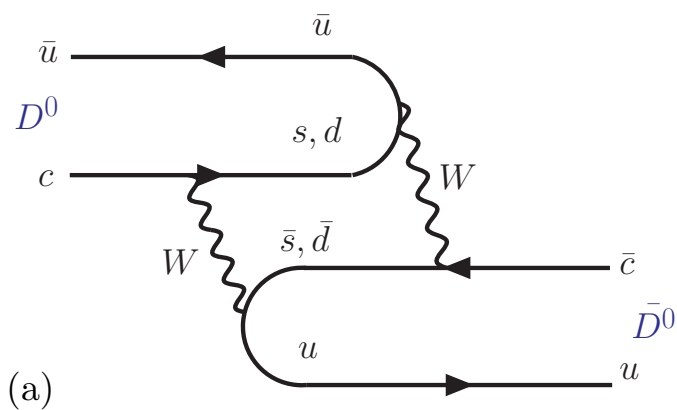

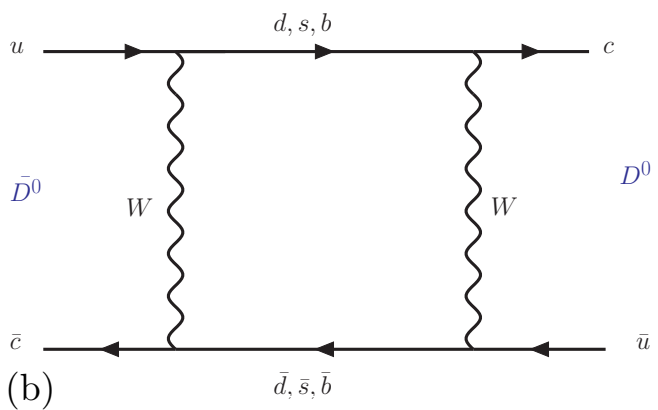

(b)

Figure 1.2. In long range processes (a), a $D^{0}$ evolves into an intermediate state such as $K^{+} K^{-}$or $\pi^{+} \pi^{-}$and then into $\bar{D}^{0}$ which subsequently decays into $K^{-} \pi^{+}$as Cabibbo favored decay. In short range processes (b) a $\bar{D}^{0}$ is shown decaying into $D^{0}$ through the box diagram.

new physics, such as fourth generation quark, appearing in the box diagram can enhance $D^{0}-\overline{D^{0}}$ mixing.

The contributions from the long-range processes are proportional to the mass of strange quark $\left(m_{s}\right)$ through $\mathrm{SU}(3)$ symmetry breaking, with

$$
x, y \approx \sin ^{2} \theta_{\mathrm{C}} \times \mathrm{m}_{\mathrm{s}}^{2},
$$

where $\theta_{C}=\tan ^{-1} \frac{\left|\mathrm{V}_{\mathrm{us}}\right|}{\left|\mathrm{V}_{\mathrm{ud}}\right|} \approx 12.7^{\circ}$, is the Cabibbo angle. Recent calculations from long range processes predict $x, y \approx \mathcal{O}\left(10^{-2}\right)[7]$.

\subsection{Measurement of Mixing Parameters}

The $D^{0}-\bar{D}^{0}$ mixing parameters $x$ and $y$ are measured in several ways such as, $D^{0}$ decays to hadronic final states (e.g. $K^{+} \pi^{-}$and $K^{-} \pi^{+}$), $D^{0}$ decays to CP-even and non-CP eigenstates, semileptonic decays, time dependent Dalitz plot analysis, and quantum-correlated final states. This section gives a brief overview of these methods. For detailed discussion please refer to $[8,9,11]$.

\subsection{1 $D^{0}$ decay to CP-mixed hadronic final states}

An example of $D^{0}$ hadronic decay is $D^{0} \rightarrow K \pi$. In this decay channel, the time dependent ratio of DCS $\left(D^{0} \rightarrow K^{+} \pi^{-}\right)$and $\mathrm{CF}\left(D^{0} \rightarrow K^{-} \pi^{+}\right)$decay rates is measured. The DCS and CF decays are identified by using "flavor tagging" method in which the pions from $D$ 
decays are tagged.

In this method, it is not possible to measure $x$ and $y$ directly due to an unknown strong phase difference $\delta$ between the DCS and CF amplitudes. This phase needs to be determined independently. The ratio of DCS to CF decay rates, assuming no CP violation, can be approximated [8] as

$$
r(t / \tau)=R_{D}+\sqrt{R_{D}} y^{\prime}(t / \tau)+\frac{\left(x^{\prime 2}+y^{\prime 2}\right)}{4}(t / \tau)^{2},
$$

where $R_{D}$ is the squared modulus of the ratio of DCS to CF amplitudes $R_{D}=\left|\frac{A(D C S)}{A(C F)}\right|^{2}$, $x^{\prime}$ and $y^{\prime}$ are the rotated $x$ and $y$ by strong phase difference $\delta$ between the DCS and CF amplitudes as $x^{\prime}=x \cos \delta+y \sin \delta$ and $y^{\prime}=-x \sin \delta+y \cos \delta, \tau$ is the mean $D^{0}$ lifetime and $t$ is proper decay time. This formula nicely separates DCS decays from mixing. The first two terms correspond to DCS decay and the non-zero values of $x^{\prime 2}$ and $y^{\prime}$ in the last term correspond to $D^{0}-\bar{D}^{0}$ mixing.

\subsection{2 $D^{0}$ decays to CP-even and non-CP eigenstates}

This method compares the decay time distribution of CP-even and non-CP eigenstates. For example in $D^{0} \rightarrow K^{+} K^{-}$and $D^{0} \rightarrow \pi^{+} \pi^{-}$(CP-even) decays and $D^{0} \rightarrow K^{-} \pi^{+}$decays. The parameter measured is

$$
y_{C P}=\frac{\tau\left(D^{0} \rightarrow K^{-} \pi^{+}\right)}{\tau\left(D^{0} \rightarrow f_{C P}\right)}-1=y \cos \phi-\frac{1}{2} A_{m} \sin \phi
$$

where $f_{C P}$ is the $K^{+} K^{-}$or $\pi^{+} \pi^{-}$final states, the CP violating phase $\phi$ is due to the interference between mixing and decays, and $A_{m}$ is the real valued parameter describing $\mathrm{CP}$ violation in mixing amplitude. In the absence of $\mathrm{CP}$ violation, $\phi=A_{m}=0$, gives $y_{C P}=y$. The $\mathrm{CP}$ violation can be studied by comparing the decay time distributions of CP-even and CP-odd final states.

\subsubsection{Semileptonic decays}

The decay $D^{0} \rightarrow K \mu \nu$ is an example of a $D^{0}$ semileptonic decay. In this decay channel, in the limit of $\mathrm{CP}$ conservation, the time integrated mixing rate relative to time integrated 
right sign decay rate $\left(R_{M}\right)$ is measured. The relation between $R_{M}$ and $x, y$ is given by

$$
R_{M}=\int r(t) d t=\frac{1}{2}\left(x^{2}+y^{2}\right)
$$

\subsubsection{Time dependent Dalitz plot analysis}

Multibody hadronic decays such as $D^{0} \rightarrow K_{S} \pi^{+} \pi^{-}$can be analyzed with the Dalitz analysis method. The individual contributions of CF decay $\left(D^{0} \rightarrow K^{*-} \pi^{+}\right)$, DCS decay $\left(D^{0} \rightarrow\right.$ $K^{*+} \pi^{-}$), and CP eigenstates (e.g $D^{0} \rightarrow \rho^{0} K_{S}$ ) can be identified by analyzing their Dalitz plots. The time evolution of the Dalitz plot is used to measure the mixing parameters.

\subsubsection{Quantum-correlated final states}

Heavy quarkonium resonances produce CP correlated meson-anti meson pairs, for example $\psi(3770) \rightarrow D^{0} \bar{D}^{0}$. The effective branching ratio of correlated decays are modified with respect to uncorrelated $D^{0}$ decays. The parameters $R_{M}$ and $\sqrt{R_{D}} \cos \delta$ can be measured, for example in $\psi(3770) \rightarrow D^{0} \bar{D}^{0}$ channel, by measuring the branching ratio of one $D^{0}$ decay into a $\mathrm{CP}$ eigenstate and the other $D^{0}$ decay into a semileptonic final state.

\subsection{Experimental Status}

$D$ mesons were discovered in 1976. Since then, physicists have searched for neutral $D$ meson mixing. Thirty-one years after the discovery, the first evidence for the $D^{0}-\bar{D}^{0}$ mixing was provided by the BaBar and Belle experiments in March 2007, at the Moriond conference $[18,20,19]$. The BaBar result was soon confirmed by the CDF experiment and announced at the Lepton-Photon conference in August 2007 [21, 22].

BaBar analyzed the difference in decay time distributions of $D^{0} \rightarrow K^{+} \pi^{-}$and $D^{0} \rightarrow$ $K^{-} \pi^{+}$decays in the PEP-II storage rings at SLAC with $384 \mathrm{fb}^{-1}$ data. The results are shown in Figure 1.3. The plot shows the projections of the proper-time distributions of combined $D^{0}$ and $\bar{D}^{0}$ wrong sign candidates. They fit this data in two ways: with mixing and without allowing mixing, and with no $\mathrm{CP}$ violation. The two fits are overlaid as solid (with mixing) and dashed (without mixing) lines. The bottom plot shows the difference 


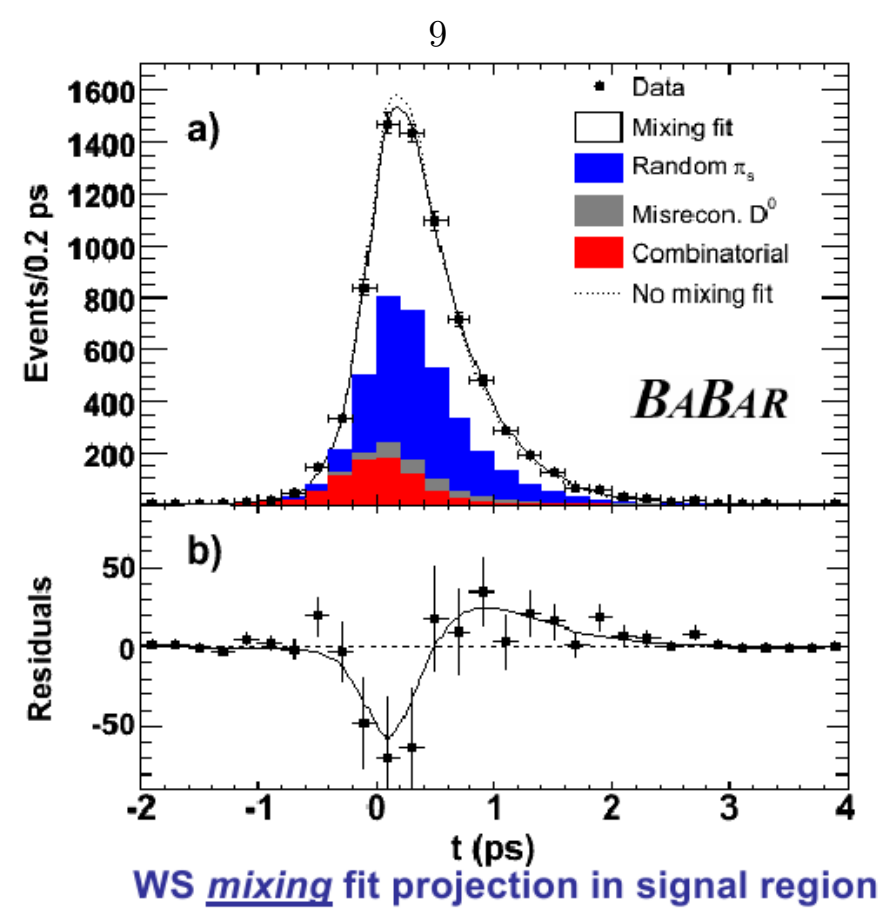

Figure 1.3. The BaBar result. The BaBar experiment compares the decay time distributions of $D^{0} \rightarrow K^{+} \pi^{-}$and $D^{0} \rightarrow K^{-} \pi^{+}$. The plot shows combined $D^{0}$ and $\bar{D}^{0}$ WS candidates with and without mixing fits (with no $\mathrm{CP}$ violation). The residual plot is the difference between the two fits and the structure appearing in that plot corresponds to $D^{0}-\bar{D}^{0}$ mixing [19].

between the two fits (residuals). The structure appearing in the residual plot corresponds to $D^{0}-\bar{D}^{0}$ mixing. Their result excludes no mixing with 3.9 Gaussian standard deviations. This result was confirmed by CDF by analyzing the same decay channel. The CDF result excludes mixing with 3.8 Gaussian standard deviations.

The Belle experiment compares decay time distributions of $D^{0} \rightarrow K^{+} K^{-}$and $D^{0} \rightarrow$ $\pi^{+} \pi^{-}$decays with $D^{0} \rightarrow K^{-} \pi^{+}$decays, to measure $y_{C P}$ given by Equation 1.3. Figure 1.4 shows the Belle result using $540 \mathrm{fb}^{-1}$ of data recorded at the KEKB asymmetric energy $e^{+} e^{-}$collider. The results of the simultaneous fits to decay time distributions of the selected $D^{0} \rightarrow K^{+} K^{-}, D^{0} \rightarrow K^{-} \pi^{+}$, and $D^{0} \rightarrow \pi^{+} \pi^{-}$candidates are shown in Figure 1.4(a), (b), and (c) respectively. The fits were performed with $y_{C P}$ as a common free parameter. The shaded areas are the backgrounds. Figure 1.4(d) shows the ratio of decay time distributions between the CP-even and non-CP $\left(D^{0} \rightarrow K^{-} \pi^{+}\right)$decays. The slight differences in the fit results corresponds to $D^{0}-\bar{D}^{0}$ mixing. The result excludes no mixing with 3.2 Gaussian standard deviations. This result was confirmed by BaBar in December 2007 [23]. 
10
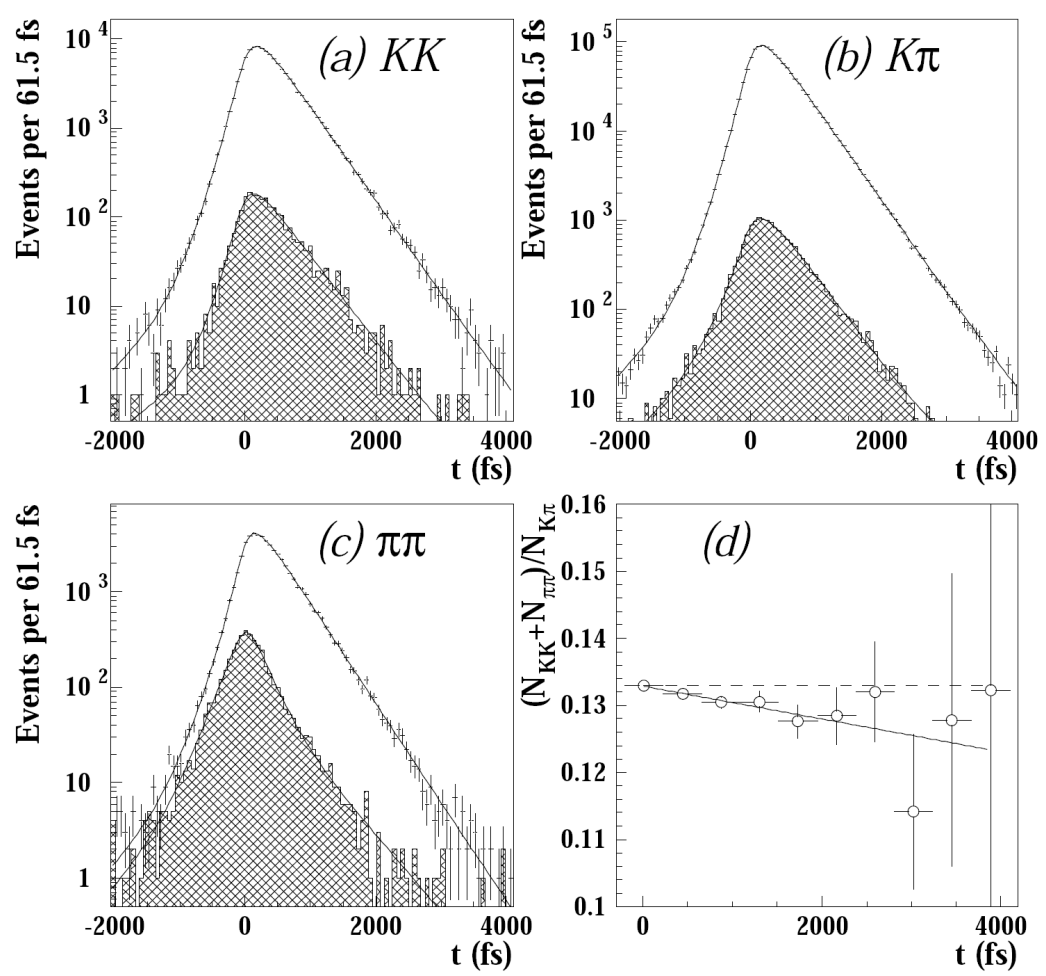

Figure 1.4. The Belle results. The plots show simultaneous fits to decay time distributions of the selected (a) $D^{0} \rightarrow K^{+} K^{-}$, (b) $D^{0} \rightarrow K^{-} \pi^{+}$, and (c) $D^{0} \rightarrow \pi^{+} \pi^{-}$candidates. The fits were performed with $y_{C P}$ as a common free parameter. The shaded areas are the backgrounds. Plot (d) is the ratio of decay time distributions between the $\mathrm{CP}$ even and non-CP decays [20].

Other charm mixing measurements from semileptonic decays [24], multi-pion or two body and three body hadronic decays [25], and time dependent Dalitz plot analyses [26, 27] are of 1 to $3 \sigma$ in significance. The CLEO experiment provided the measurement of the mixing parameter $y$ and strong phase difference $\delta$ using $\psi(3770) \rightarrow D^{0} \overline{D^{0}}$ channel [28].

Figure 1.5 shows the evidence for $D^{0}-\bar{D}^{0}$ mixing from the BaBar and CDF experiments published in 2007. The measurements of the mixing parameters are listed in Table 1.1. Figure 1.6 shows $1-\sigma$ contours (standard error ellipses) in $x^{2}-y^{\prime}$ space allowing the result to be compared for consistency. These contours are based on the fit error matrices of the BaBar, Belle, and CDF (2007) results. The ellipses are drawn using the formula:

$$
\frac{(x-a)^{2}}{\sigma_{x}^{2}}-\frac{2 \rho(x-a)(y-b)}{\sigma_{x} \sigma_{y}}+\frac{(y-b)^{2}}{\sigma_{y}^{2}}=1-\rho^{2},
$$




\begin{tabular}{|c|c|c|c|}
\hline \hline Experiment & $R_{D}\left(\times 10^{-3}\right)$ & $y^{\prime}\left(\times 10^{-3}\right)$ & $x^{\prime 2}\left(\times 10^{-3}\right)$ \\
\hline BaBar & $3.03 \pm 0.16 \pm 0.10$ & $9.7 \pm 4.4 \pm 3.1$ & $-0.22 \pm 0.30 \pm 0.21$ \\
Belle & $3.64 \pm 0.17$ & $0.6 \pm 4.0 \pm 3.9$ & $0.18 \pm 0.21 \pm 0.23$ \\
CDF & $3.04 \pm 0.55$ & $8.54 \pm 7.55$ & $-0.12 \pm 0.35$ \\
\hline \hline
\end{tabular}

Table 1.1. Current values of $D^{0}-\bar{D}^{0}$ mixing parameters provided by BaBar, Belle, and CDF experiments from $D^{0} \rightarrow K \pi$ decays.

where $x$ and $y$ are the points in $x^{\prime 2}-y^{\prime}$ space, $a$ is the measured value of $x^{\prime 2}, b$ is the measured value of $y^{\prime}, \sigma_{x}$ is the uncertainty on $x^{\prime 2}, \sigma_{y}$ is the uncertainty on $y^{\prime}$, and $\rho$ is the covariance coefficient defined as $\rho=(<x y>-<x><y>) / \sigma_{x} \sigma_{y}$. The values of $a, b, \sigma_{x}$, and $\sigma_{y}$ are given in Table 1.1. Covariances for the BaBar and Belle results were quoted in their publications as $\rho_{\text {BaBar }}=-0.94$ and $\rho_{\text {Belle }}=-0.909$. For the CDF II (2007) result, the covariance $\rho_{C D F}$ was -0.98 .

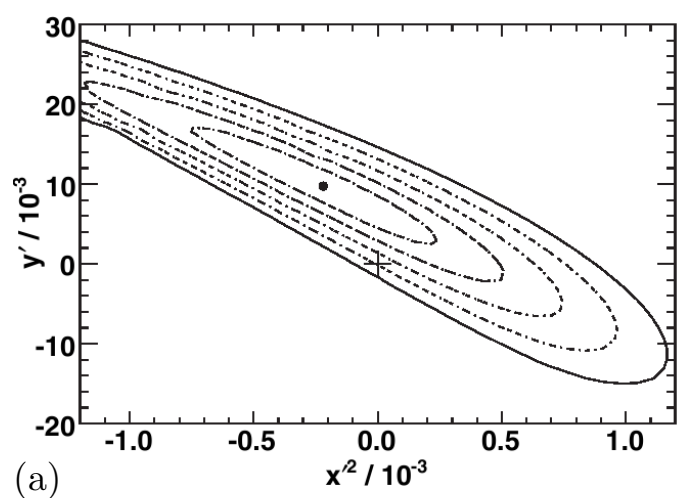

(a)

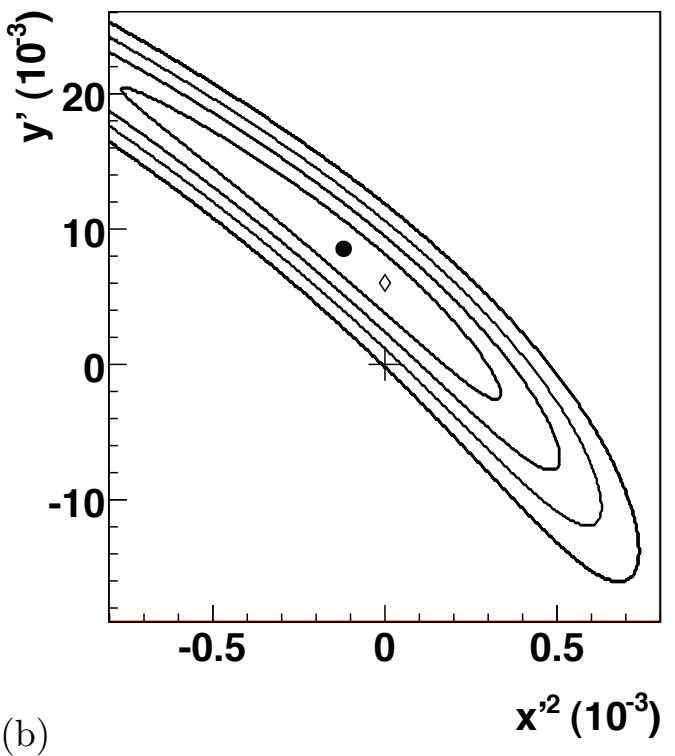

Figure 1.5. Evidence for $D^{0}-\overline{D^{0}}$ mixing. (a) Babar result $\left(384 \mathrm{fb}^{-1}\right)$. The $1-5 \sigma$ contours exclude no-mixing at $3.9 \sigma$ level [19]. (b) CDF result $\left(1.5 \mathrm{fb}^{-1}\right)$. The Bayesian probability contours equivalent to $1-4 \sigma$. No-mixing point is excluded at $3.8 \sigma$ level [22].

By combining all the published results and performing a $\chi^{2}$ fit on the related observables, the Heavy Flavor Averaging Group (HFAG) provided the world average value of the $D^{0}-$ $\overline{D^{0}}$ mixing significance as 9.2 Gaussian standard deviations [29]. Figure 1.7 shows a two dimensional contour plots for mixing parameters $x$ and $y$. HFAG provided the values of $x$ 


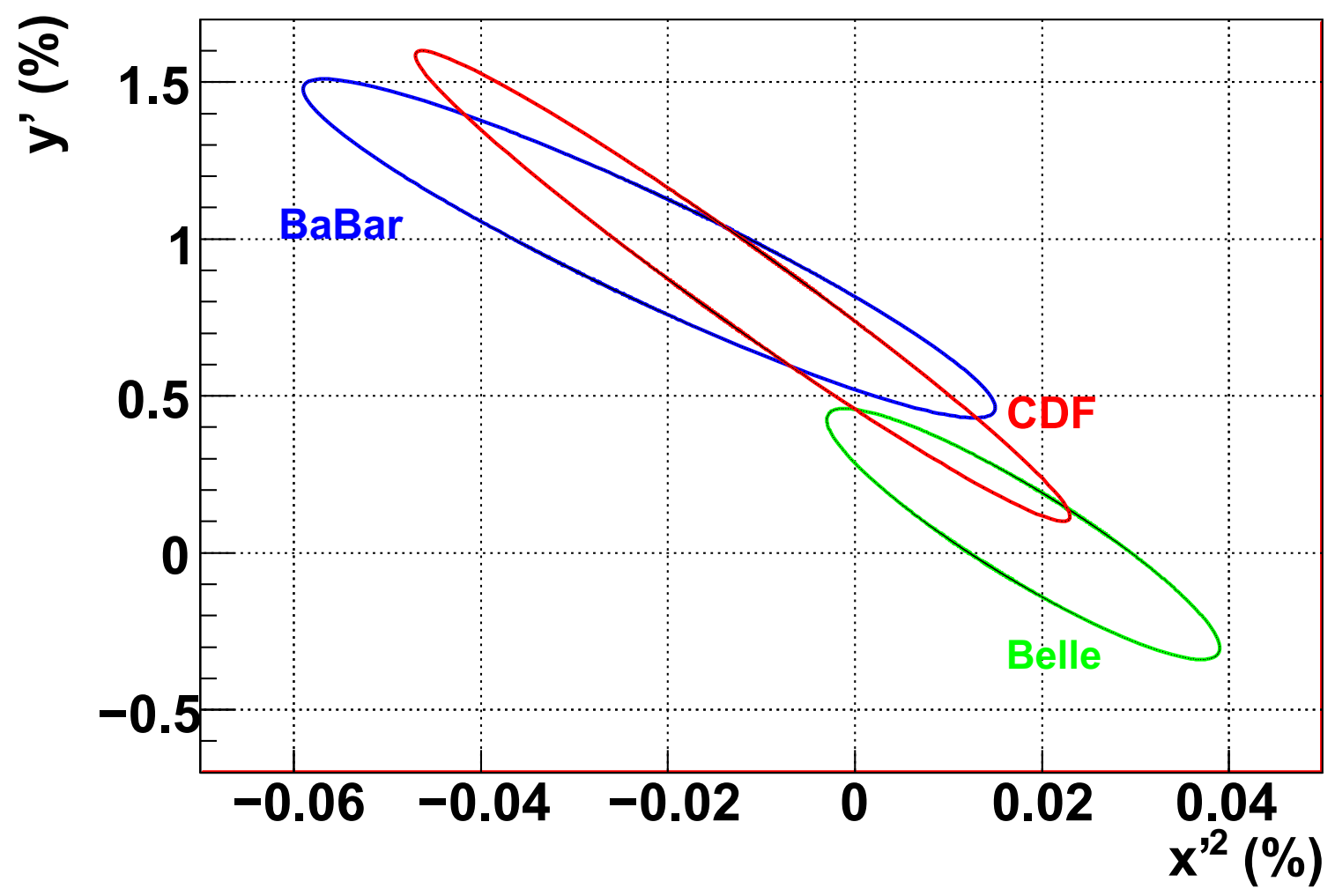

Figure 1.6. Visual comparison of Belle (green), BaBar (blue), and CDF (2007) (red) measurements. These are the standard error ellipses based on the fit error matrices of the three results. The BaBar measurements are centered around $x^{\prime 2}=-0.022 \%, y^{\prime}=0.97 \%$. The Belle measurements are centered around $x^{\prime 2}=0.018 \%, y^{\prime}=0.06 \%$. The CDF (2007) measurements are centered around $x^{2}=-0.012 \%, y^{\prime}=0.85 \%$.

and $y$ as

$$
x=0.89 \pm 0.26 \pm 0.27 \text { and } y=0.75 \pm 0.17 \pm 0.18 \text {. }
$$

The world average significance clearly represents $D^{0}-\bar{D}^{0}$ mixing from the combination of all published results. However, mixing was not been observed in a single decay channel with more than $5 \sigma$ significance. There is also no evidence for $\mathrm{CP}$ violation.

The results presented in this thesis will show the observation of $D^{0}-\overline{D^{0}}$ mixing in $D^{0} \rightarrow K \pi$ channel using the CDF II detector. 


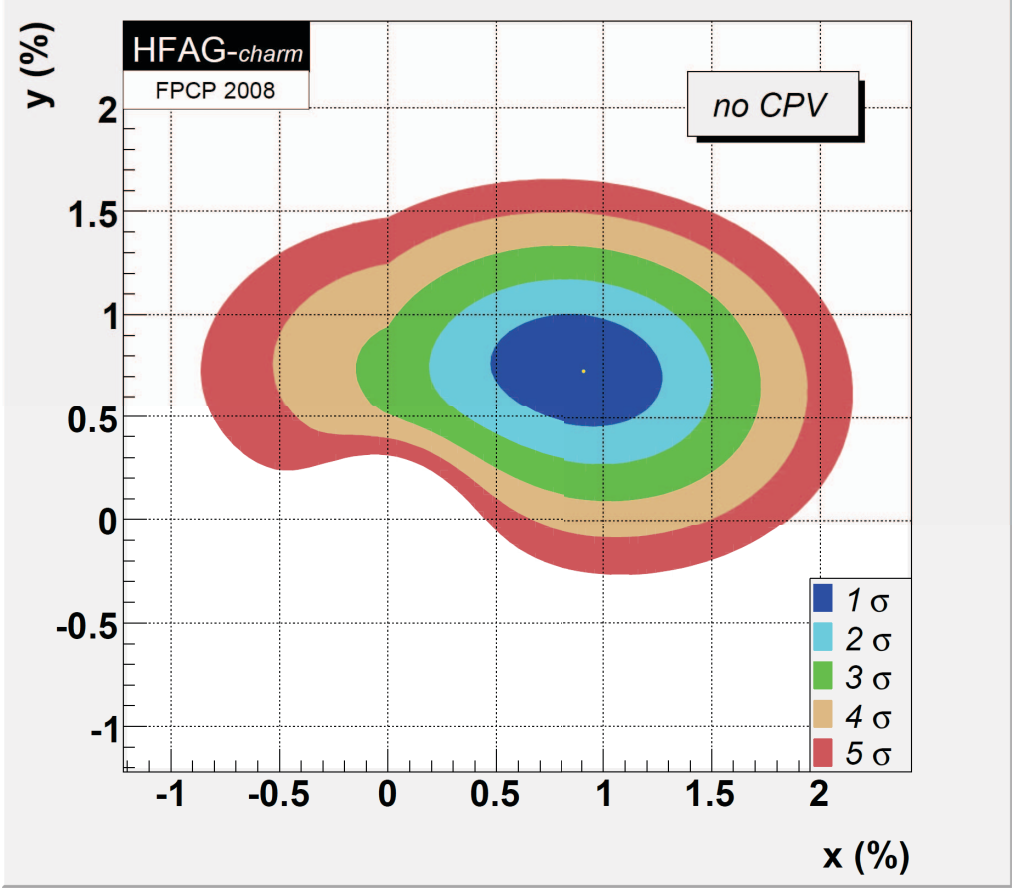

Figure 1.7. Two dimensional contours for mixing parameters $x$ and $y$ provided by heavy flavor averaging group [29]. 


\section{CHAPTER 2}

\section{EXPERIMENTAL SETUP}

"What is known about a collision is what goes in and what comes out-and how it comes out. What happens in that tiny volume of the collision? The maddening truth is that we can't see it... We see what goes in and what comes out, and we ask if the patterns are predictable by our model of what is in box."

- Leon M. Lederman, The God Particle (1993).

Oscillations of neutral $D$ mesons are detected using the CDF II detector. The CDF II detector observes the products of proton-anti proton collisions at the Tevatron accelerator at Fermilab. This chapter describes the Tevatron accelerator and the components of the CDF II detector that are relevant to this analysis.

\subsection{The Tevatron}

The Tevatron is a high energy particle accelerator that collides protons and anti-protons at the center of mass energy of $\sqrt{s}=1.96 \mathrm{TeV}$. The Tevatron is a superconducting synchrotron of radius $1 \mathrm{~km}$ and is a part of the Fermilab accelerator chain [30]. A sketch of the Fermilab accelerator chain is shown in Figure 2.1. The following text gives a simplified description of the accelerator.

A synchrotron uses a time-varying magnetic field to maintain a constant orbital radius for the charged particles while the energy of the particles increases as they are accelerated by radio frequency $(\mathrm{RF})$ cavities. Protons are produced by ionizing hydrogen gas using an electric discharge in the Cockcroft-Walton accelerator. The hydrogen ions $\left(H^{-}\right)$are accelerated up to an energy of $750 \mathrm{KeV}$ before they are injected into a 130 meter long linear accelerator called the Linac. A series of RF cavities in the Linac accelerates the hydrogen ions to an energy of $400 \mathrm{MeV}[32,33]$. The ion beam passes through a carbon foil which strips off electrons to produce protons. The protons are injected into a smaller rapid cycling synchrotron called the booster. The booster is about 150 meters in diameter. The protons 


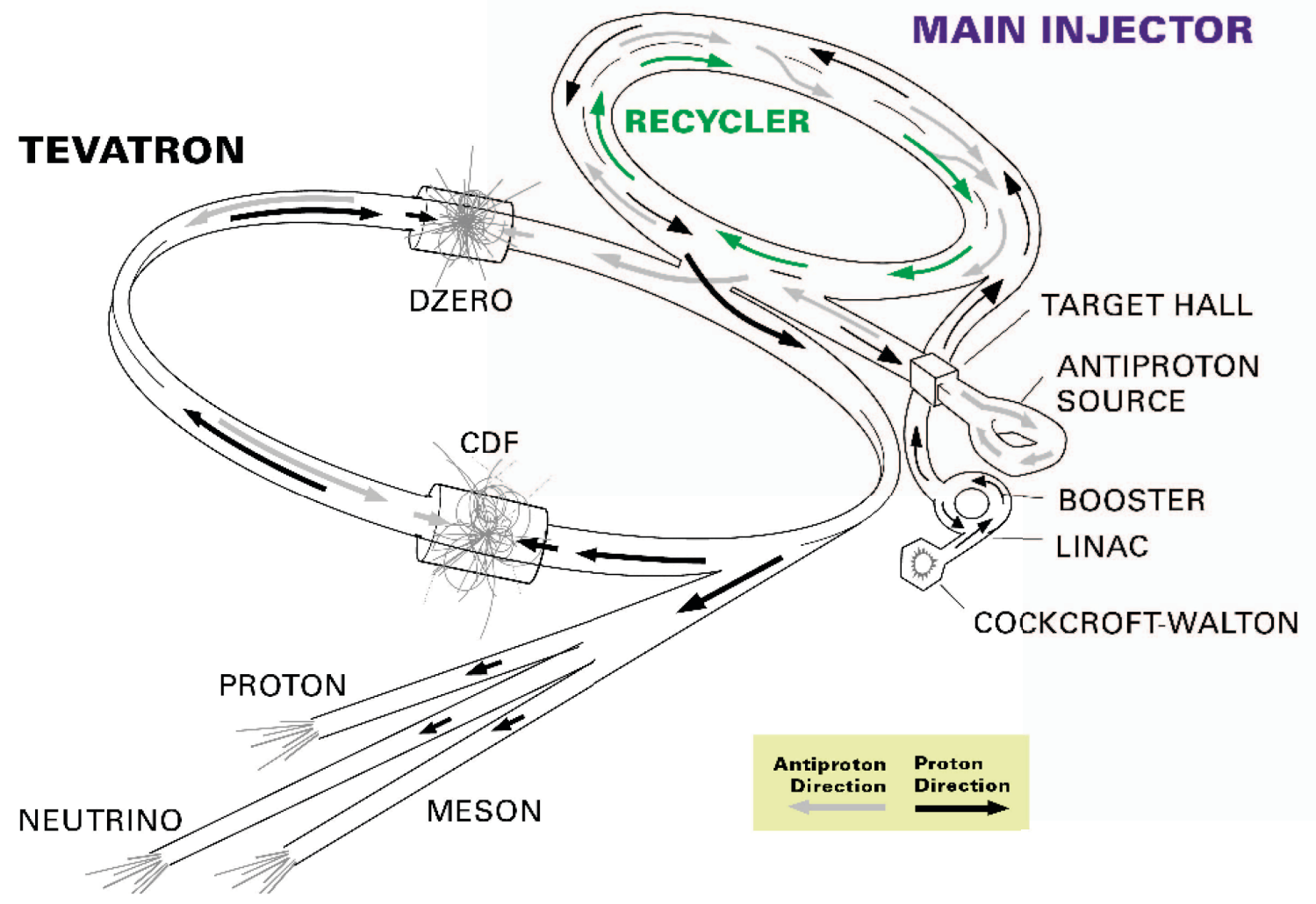

Figure 2.1. Fermilab accelerator chain [30, 31].

travel through the ring multiple times, gaining an energy of $8 \mathrm{GeV}$ [34]. Then the protons are transferred to the main injector, a synchrotron of about $1 \mathrm{~km}$ in diameter. The main injector accelerates the protons up to an energy of $150 \mathrm{GeV}$ before injecting them into the Tevatron ring [35].

At the main injector, some proton bunches of energy $120 \mathrm{GeV}$ are diverted into the Target Hall to strike a nickel target in order to produce anti-protons [36]. This is done at 1.5 second intervals. Since a relatively small number of anti-protons are produced in each cycle, they need to be accumulated in a storage ring until a sufficient number is reached. The density of anti-protons is increased using the electron cooling method. In this method, a beam of electrons travels in parallel with the anti-proton beam. During this process, some of the transverse energy of the anti-protons is transferred to the electron beam. When enough anti-protons are collected, they are transferred back to the main injector and accelerated up to an energy of $150 \mathrm{GeV}$ before they are injected in the Tevatron ring. 
At the Tevatron, over a thousand superconducting dipole magnets guide the beam around the ring. Anti-protons are injected after the protons are loaded into the Tevatron. Once the Tevatron loading is complete, the particles are accelerated to an energy of 980 $\mathrm{GeV}$. The protons and anti-protons run in 36 bunches each. A proton bunch contains about $2.6 \times 10^{11}$ protons, while an anti-proton bunch contains about $3.5 \times 10^{10}$ anti-protons. The proton anti-proton bunches cross at intervals of $396 \mathrm{~ns}$. The protons and anti-protons travel in non-intersecting helices. At the collision point, the beams are focused using quadrupole magnets and the beam spot size is reduced to increase the luminosity.

The instantaneous Tevatron luminosity is given by:

$$
\mathcal{L}=\frac{N_{B} N_{p} N_{\bar{p}} f}{2 \pi\left(\sigma_{p}^{2}+\sigma_{\bar{p}}^{2}\right)},
$$

where, $N_{B}$ is the number of bunches, $N_{p}$ is the number of protons, $N_{\bar{p}}$ is the number of anti-protons, $f$ is bunch revolution frequency, and $\sigma_{p}$ and $\sigma_{\bar{p}}$ are effective widths of proton and anti-proton beams.

The time integrated luminosity is measured in the units of inverse femtobarn $\left(\mathrm{fb}^{-1}\right)$, where one barn is equivalent to $10^{-24} \mathrm{~cm}^{2}$.

In the beginning, when the collisions start with a new store, the initial luminosity is quite high. As particles are lost over time, the instantaneous luminosity decreases. After the accelerator runs for about 15 hours, the beam is terminated. By this time, enough new anti-protons are accumulated to start a new cycle. One such cycle is called a "store".

\subsection{The CDF II Detector}

CDF II is a general purpose detector designed to accommodate many of the particle physics programs such as heavy flavor, electroweak, top, QCD, Higgs, and Exotic and other new physics searches. The Roman number II in the name of the detector symbolizes the upgraded detector system. Figure 2.2 shows a picture of the CDF II detector. A schematic view of the detector complex is shown in Figure 2.3.

In the coordinate system of the CDF II detector, the $z$-axis is along the beam line with its origin at the center of the detector. The positive $z$-axis is chosen to be in the 


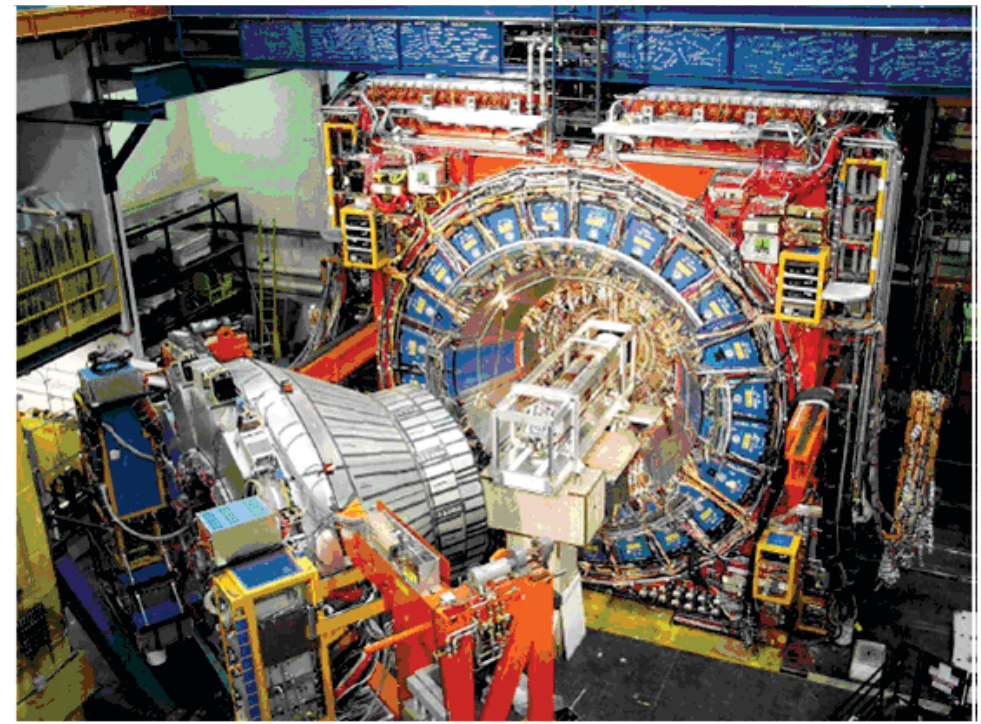

Figure 2.2. A picture of CDF II detector with end plug opened [31, 37].

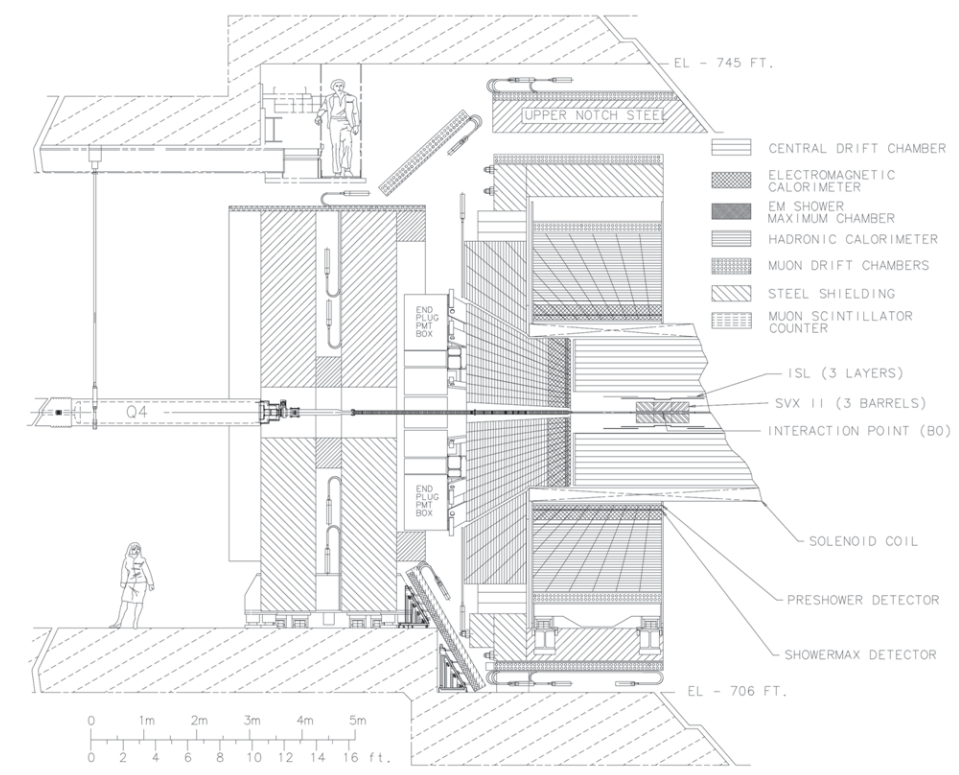

Figure 2.3. Elevation view of the CDF II detector [31, 37].

direction of the proton beam. The $x$-axis and $y$-axis are chosen to make it a right-handed coordinate system with vertical $y$-axis. The $x$-axis points horizontally to the center of the ring. Sometimes, a cylindrical coordinate system is also considered due to the cylindrical shape of the detector. In this system, $r$ is the radius, $\phi$ is the azimuthal angle measured from the positive $x$-axis; and the $z$-axis is the same as that in Cartesian geometry. In the polar coordinate system, $\theta$ is measured from the positive $z$-axis. Sometimes, $\theta$ is replaced 


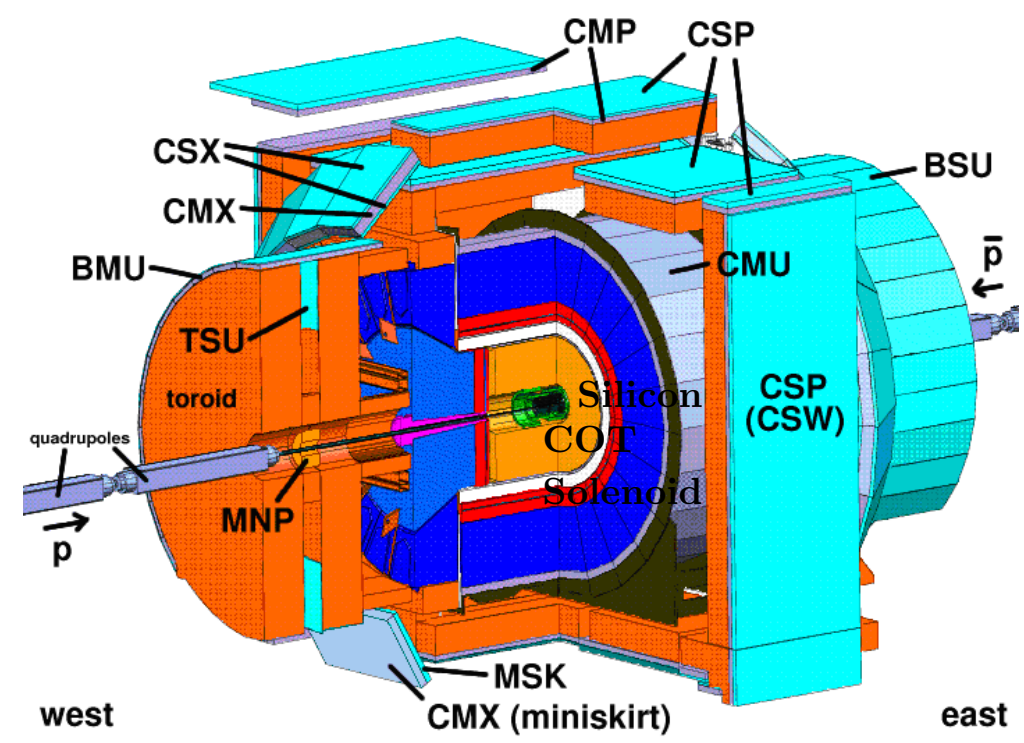

Figure 2.4. Isometric view of the CDF II detector [31, 37].

by pseudo-rapidity $\eta$ defined as $\eta=-\ln \left(\tan \frac{\theta}{2}\right)$.

The most probable collision point is at the center of the detector. The collision region is surrounded by various detector systems as shown in Figure 2.4. In our analysis, we reconstruct charged particle tracks using the tracking and vertexing systems, a silicon detector and a multi-wire drift chamber called the "Central Outer Tracker" or "COT". These two components are described in the following text. A comprehensive description of the complete system can be found in reference [37]. The following discussion is based on references $[39,40,41,42]$.

The $r-z$ view of the CDF II tracking and vertexing system is shown in Figure 2.5. The tracking system is surrounded by a 4.8 meter long cylindrical superconducting solenoid with a diameter of 3 meters and axis along the $z$-axis. The solenoid produces a uniform magnetic field of 1.4 T. A charged particle travelling in this magnetic field forms a helical trajectory that is characterized by the charge and momentum of the particle. The projection of the helix on the transverse plane (the plane perpendicular to beam line) is a circle of radius $r$. The curvature $C$ of the helix is defined as $|C|=q / 2 r$, where $q$ is the charge of the particle. A helical fit is performed to determine the curvature of a track and other track parameters. The transverse momentum of the particle is measured from the curvature as $p_{T}=B q r$, where $B$ is the magnitude of the magnetic field. The longitudinal component of 


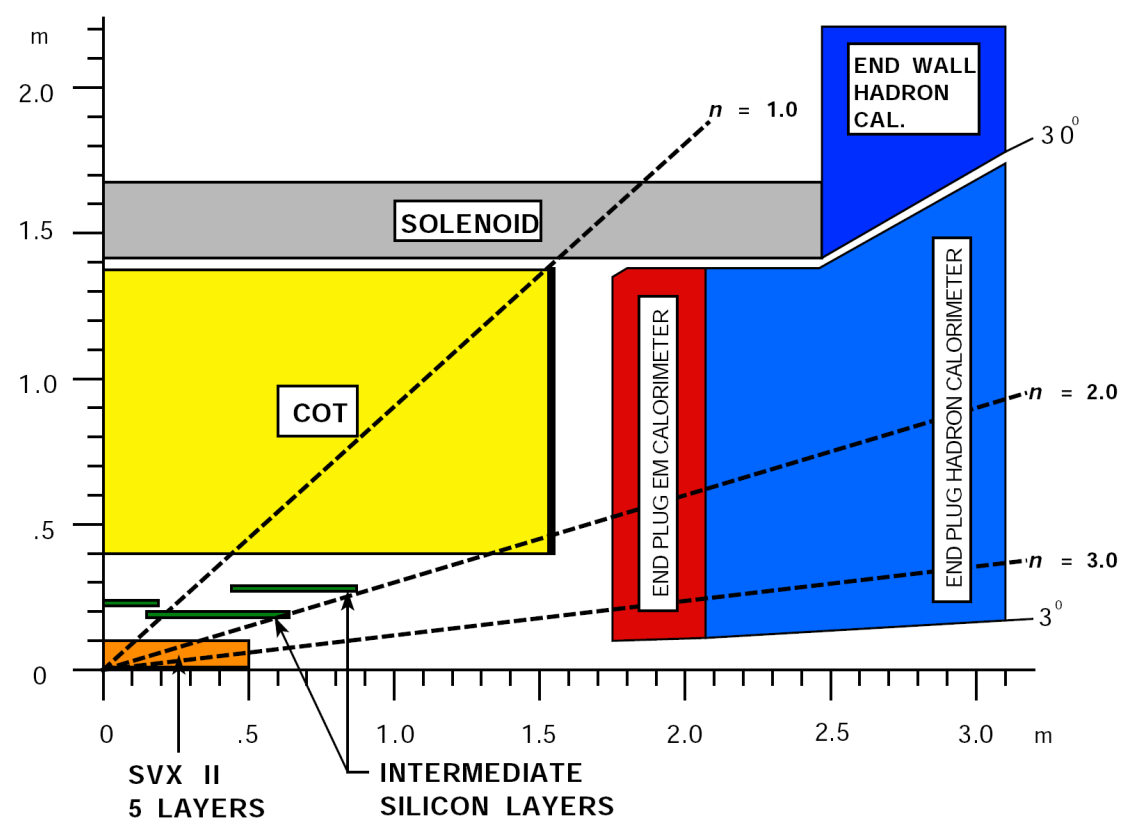

Figure 2.5. The $r-z$ view of the CDF II tracking system [31, 37].

the momentum is given by $p_{z}=p_{T} \cot \theta$. The momentum is measured in the units of $\mathrm{GeV} / c$.

A vertex is the point in the detector coordinate system at which a particle originates. A vertex is formed when a particle decays into another particle. Since the decaying particle decays into more than one particle, the decay vertex can be determined from the intersection of the trajectories of at least two decay products.

The particle trajectories are determined using the silicon detector system and the Central Outer Tracker (COT).

\subsubsection{Silicon Detector System}

The silicon detector system is the innermost part of the CDF II detector. The purpose of the silicon detector system is precision tracking and vertexing. It spans the region of $|\eta|<2.0$, as shown in Figure 2.6.

The silicon detector system comes in three subsystems as illustrated in Figure 2.7.

- L00: Immediately around the beam pipe $(r=1.6 \mathrm{~cm})$, there is a single sided silicon micro strip detector, called L00. The micro-strip array has a pitch of $50 \mu \mathrm{m}$. 
- SVX II: Outside the L00, extending from $r=2.4 \mathrm{~cm}$ to $r=10.7 \mathrm{~cm}$, is a double sided micro-strip detector called silicon vertex detector SVX II. The SVX II is arranged in 5 concentric barrels. Three of them have silicon strips positioned at a stereo angle of $90^{\circ}$ to provide information in the $z$ direction. The strips on the other two layers have a small stereo angle of $1.2^{\circ}$ making them sensitive to both $z$ and $\phi$ directions.

- ISL: Outside the SVX II is the Intermediate Silicon Layer or ISL ranging from $r=20$ $\mathrm{cm}$ to $r=29 \mathrm{~cm}$, corresponding to $1.0<|\eta|<2.0$ region. The ISL is again a set of double sided micro-strip detectors arranged in three concentric barrels. One side of the silicon detector has a strip pitch of $55 \mu \mathrm{m}$, the strip pitch on the other side is 73 $\mu \mathrm{m}$.

Figure 2.8 is a sketch to illustrate how a particle is detected in the silicon micro-strip detectors. When a charged particle passes through the silicon strips, it leaves behind electronhole pairs. An electric pulse (called hit) is detected in the nearest strips. The hit position is extracted by weighting the strip positions by the amount of charge collected on the strips. The accuracy of the silicon detector in measuring the hit position is about $12 \mu \mathrm{m}$. The particle track is reconstructed from the signals in different layers. Tracking is performed by a dedicated system called Silicon Vertex Tracker (SVT). Most of the electronics such as amplifiers and analog-to-digital converters are kept close to the silicon detectors to avoid loss of signal. This makes the system extremely sensitive to particle radiation.

\subsubsection{Central Outer Tracker}

The Central Outer Tracker is a cylindrical drift chamber located between $r=44 \mathrm{~cm}$ and $r=132 \mathrm{~cm}$. It is $310 \mathrm{~cm}$ long and spans the range of $|\eta|<1.0$. The COT is filled with a mixture of argon and ethane gases with equal concentration.

As illustrated in Figure 2.9, the COT is divided in eight superlayers (SL1 to SL8, with SL1 being the innermost and SL8 being the outermost superlayer). Each superlayer has 12 sense wires providing total 96 radial measurement points. The number of cells (peripheral divisions) in each superlayer ranges from 168 for SL1 to 480 for SL8. Superlayers 2, 4, 6, and 8 are axial (wires parallel to the beam) while superlayers $1,3,5$, and 7 are at a small 


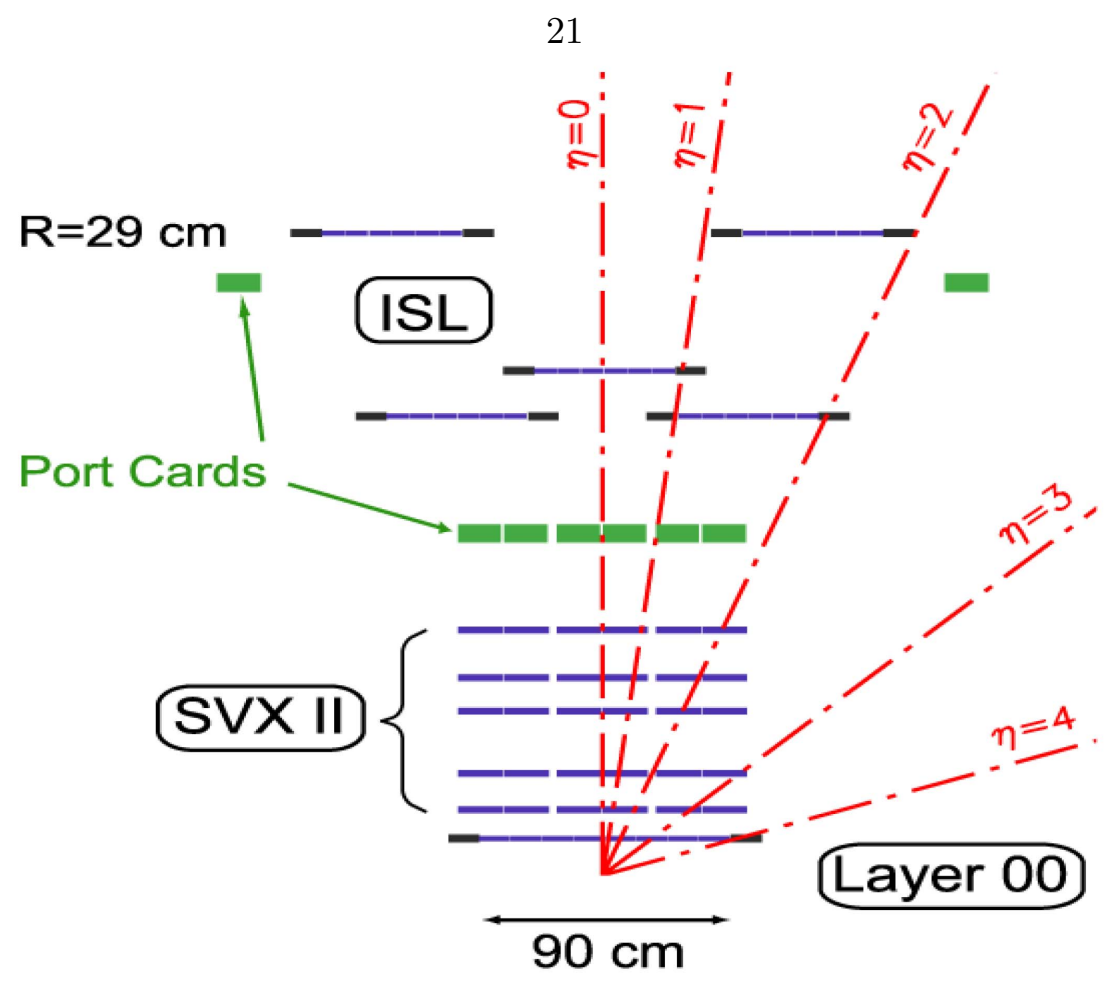

Figure 2.6. Components of silicon detector system spanning various pseudo-rapidity $(\eta)$ regions $[31,37]$.

stereo angle $\left(2^{\circ}\right)$. Thus, there are a total of 2520 cells. Figure 2.10 shows one such cell. As illustrated in the figure, the wire plane makes an angle of $35^{\circ}$ with the radial direction.

Similar to what happens in the silicon detector, a charged particle travelling through the gas mixture leaves a trail of ions. An electric field is created by cathode field panels and potential wires. This electric field causes the ions to drift toward the sense wires. Due to the combined effect of the magnetic field in the COT and the electric field in the cell, the ions drift towards the sense wire with an angle $\alpha=35^{\circ}$.

The COT has a resolution of $180 \mu \mathrm{m}$ for a single hit. COT tracks are reconstructed by a hardware system called eXtremely Fast Tracker (XFT). For the complete track reconstruction, the COT and SVX information are combined. The XFT extrapolates the COT tracks into SVX. The COT also measures the ionization energy loss $(\mathrm{dE} / \mathrm{dx})$ of a particle.

Apart from COT and SVX detectors, the CDF II also has a third detector called Muon Detector. The Muon detector is comprised of Central Muon detector (CMU), Central Muon Upgrade detector (CMP), Central Muon eXtension detector (CMX), and Intermediate Muon System (IMU). 


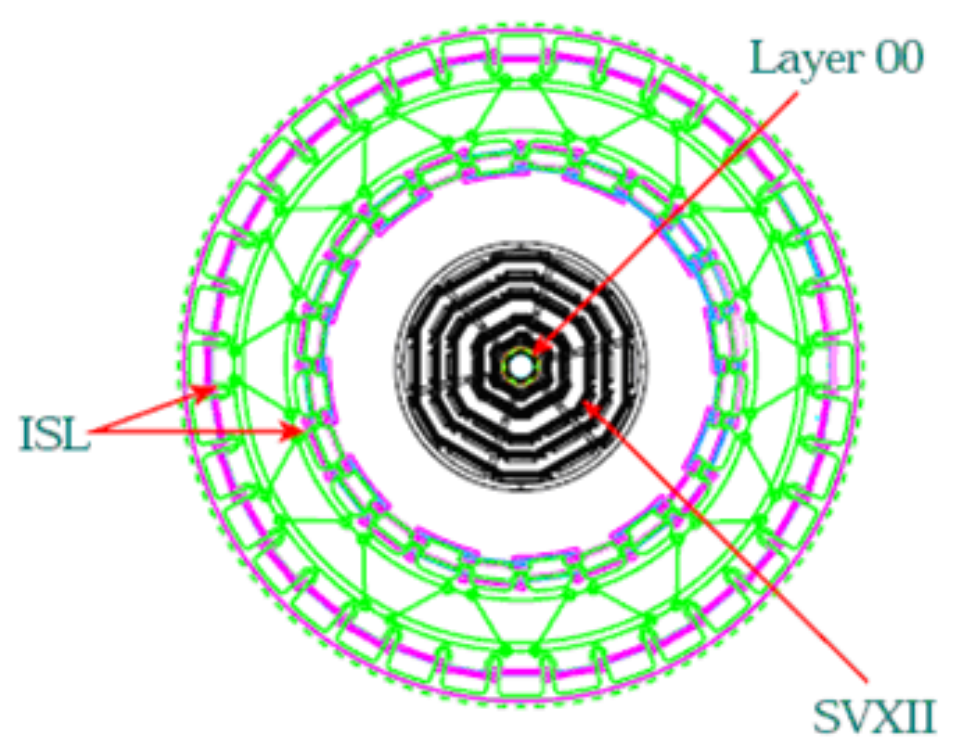

Figure 2.7. Silicon Vertex Detector end view [31, 37].

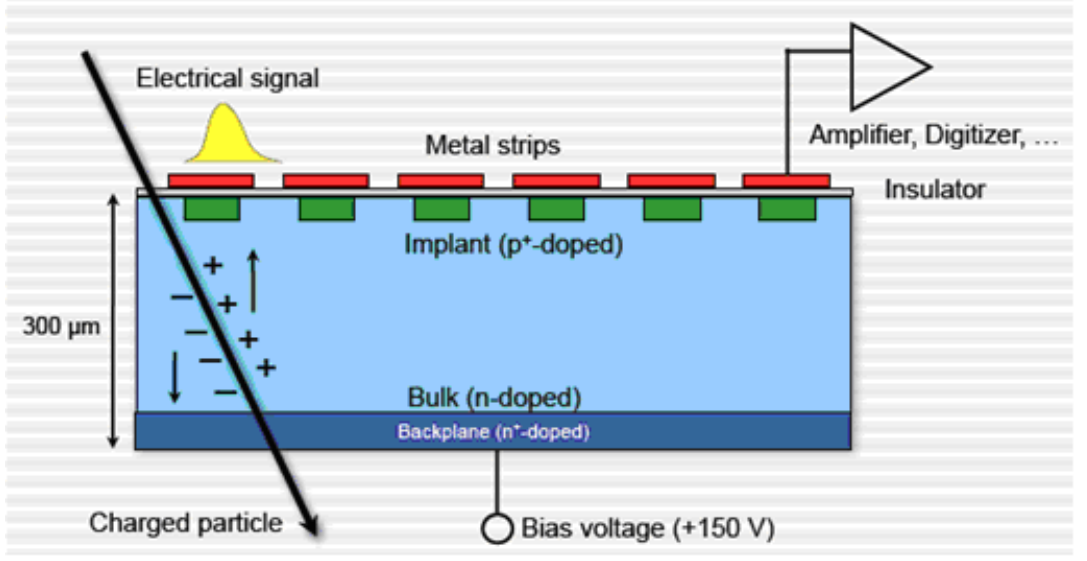

Figure 2.8. Detection of a particle track in a silicon strip detector [31, 37].

In addition, there are other components of the CDF II detector such as Cherenkov Luminosity Counter (CLC), calorimeters, and Time Of Flight (TOF) systems. The Muon detector and these components are not relevant for our analysis. For a complete review of the CDF II detector system, please refer to [37].

\subsection{Trigger System}

The bunch crossing interval of $396 \mathrm{~ns}$ at the Tevatron produces collisions at the the rate of $2.5 \mathrm{MHz}$. Only a subset of this data is of physics interest. Also, it is not feasible to 


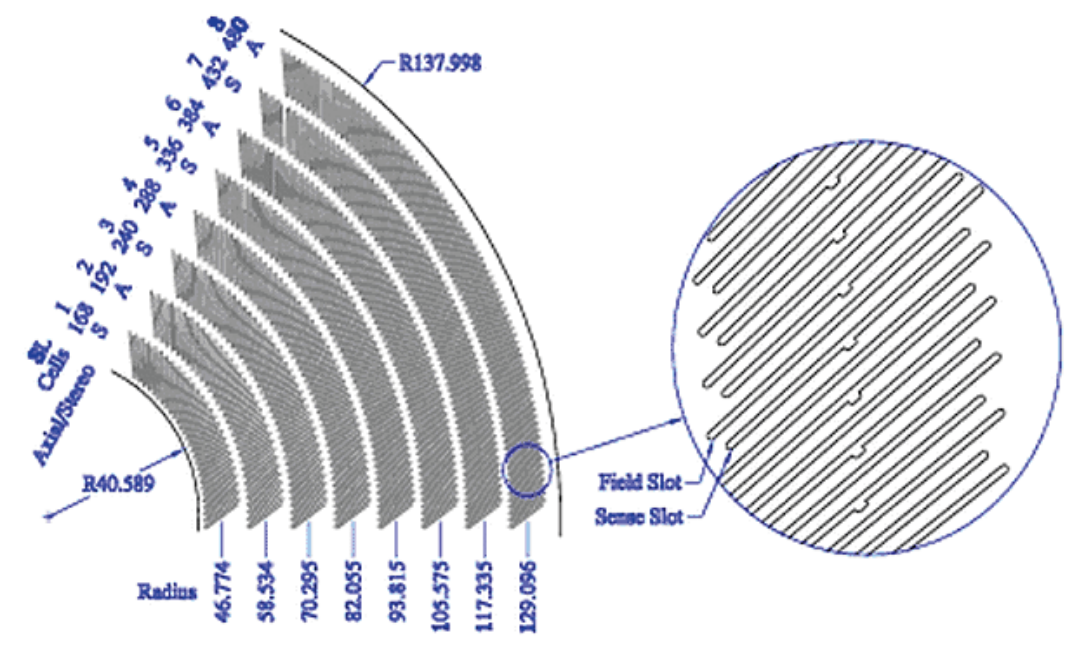

Figure 2.9. COT superlayers. COT is divided in 8 superlayers, each containing 12 wires, to provide 96 measurement points radially. The number of cells in each superlayer ranges from 168 for SL1 to 480 for SL8, providing a total of 2520 cells [31, 37].

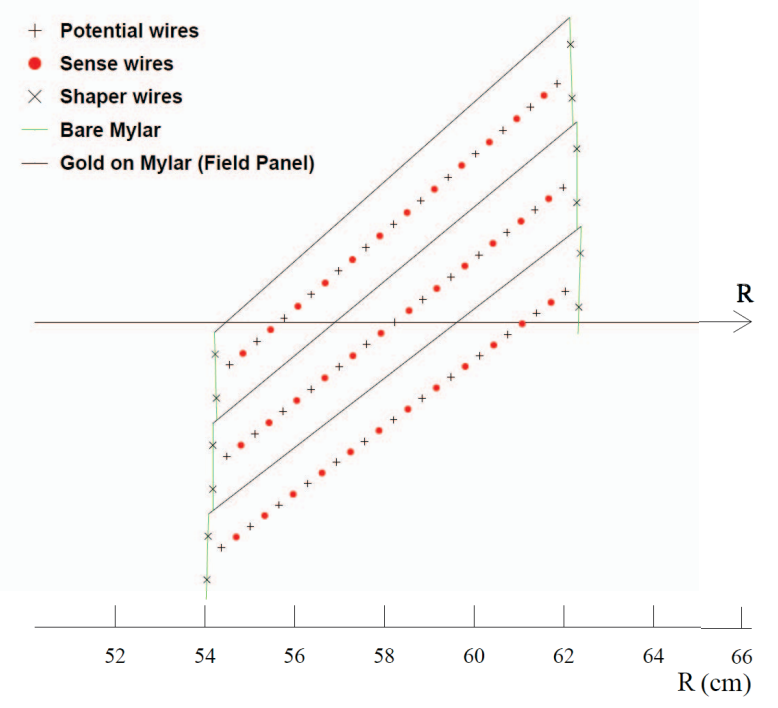

Figure 2.10. A COT cell. The wire plane makes an angle of $35^{\circ}$ with the radial direction $[31$, 37].

maintain the records of all these events in physical storage. The trigger system at CDF II is therefore designed to filter out unwanted events and only retain those events that are judged as desirable for physics analyses.

The data acquisition system at CDF II is a three level trigger system described by Level 1 (L1), Level 2 (L2), and Level 3 (L3) triggers. The triggers are designed with increasing sophistication at each level. It takes only $5.5 \mu$ s to make a judgement to accept or reject 
an event at level 1 . If an event is accepted at level 1, then further constraints are applied at level 2, which may take as much as $50 \mu$ s to make a decision.

For the triggers relevant to this analysis, at level 1, the trigger primitives are azimuthal angle $\phi$ and transverse momentum $p_{T}$ of a track. A hardware system called the "extremely fast tracker" (XFT) uses COT hits to reconstruct the particle tracks.

Level 2 is a combination of hardware and software in which the silicon vertex tracker (SVT) system uses digitized data from the SVX II detector. The SVT looks for the tracks with displaced vertices. At level 2, an important trigger primitive is the impact parameter along with Level 1 primitives.

Level 3 is a purely software trigger running on a cluster of about 300 processors. At this level, a block of data, called an event record, is formed with complete information about an event. Further constraints are applied and if the event is selected then the data are passed to permanent storage and offline processing. The final output rate at Level 3 is less than $75 \mathrm{~Hz}$. Figure 2.11 is the block diagram of the trigger system.

There are two types of triggers: pre-scaled triggers and luminosity enabled triggers. If the data rate is too high, some triggers are dynamically adjusted (prescaled) to match what the data acquisition system can handle. For example, in the beginning of a store, the initial luminosity is high. At this time, tight constraints are placed on low momentum $\left(p_{T}\right)$ tracks. When luminosity drops, these constraints are loosened to make full use of the available bandwidth. The luminosity enabled triggers are enabled only when the luminosity is within a specified range.

All triggers are stored in the CDF II trigger database. For a given trigger, the complete sequence of level 1, level 2, and level 3 requirements is called a "trigger path". This design avoids interference of triggers by separating well defined sequences of independent trigger requirements. They follow a naming convention such that an important requirement of the trigger appears in the name. For example, L1_TWO_TRK_B_HAD_LUMI_80_v2 is the trigger that looks for two oppositely charged particle tracks; it is designed for $B$ hadrons; and it is a luminosity enabled trigger that turns on after the luminosity is below $80 \times 10^{30} \mathrm{~cm}^{2} / \mathrm{s}$. The last number in the name represents the version of the trigger.

The data is divided into logical sets called "runs". Each run corresponds to a certain 


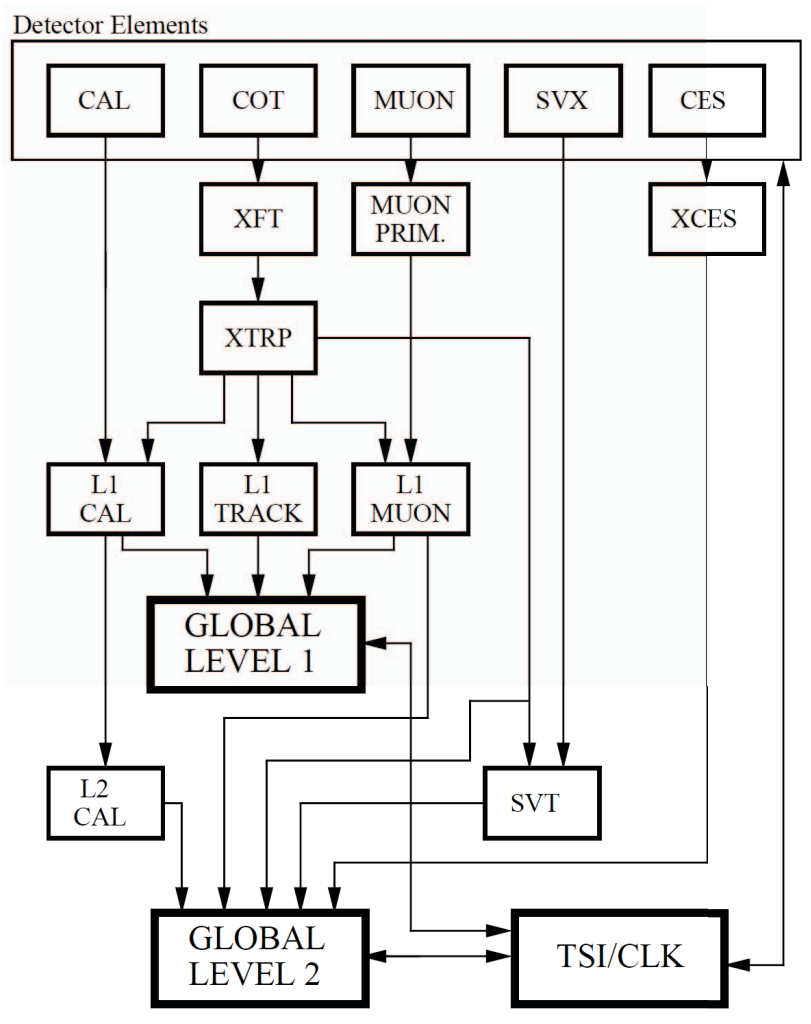

Figure 2.11. Block diagram of the three level trigger system at CDF II detector. For the purpose of this analysis, the data from COT and SVX is used. The COT data is processed by XFT (eXtremely Fast Tracker) and XTRP (Extrapolator). The SVX data is processed by Secondary Vertex Tracker (SVT). Level 2 accepts the data from Level $1[31,37]$.

time period over which the data is collected and is assigned a specific run number. A store is divided into several runs. The detector operations manager records the information about luminosity (as determined by Cherenkov Luminosity Counter or CLC) and the status of the detector components at the end of each run. This information is stored in a database called the CDF II run summary database. The runs are also marked as "good" or "bad" depending on the health of the important detector components at the time of data acquisition. The information about the total luminosity of the data used in an analysis can be obtained from this database. This is done by selecting the required components of the detector, and a range of run numbers corresponding to the used data. 


\subsection{Offline Processing}

The events accepted by Level 3 are written to tape in the mass storage system. The events are written to one of the eight data streams based on the triggers they satisfy. The tapes are cataloged in the CDF Data File Catalog, which is an Oracle database. After the necessary calibrations are applied, the data is processed on a parallel computing farm. The selected data files from tape are sent to worker nodes (the individual processors ) in the parallel computing farm. Each worker node receives a file of approximately 1 GByte in size. The events are reconstructed and the output is split into multiple $(\approx 50)$ files. Each file corresponds to a dataset defined by the event type in the trigger system. An event may satisfy several trigger requirements and can be written to multiple datasets [38].

At the end, the output files from all nodes for each dataset are combined and stored in the CDF Data Handling system (DHS). At this point, the files are accessible for various analyses. 


\section{CHAPTER 3}

\section{ANALYSIS AND RESULTS}

"Results! Why, man, I have gotten a lot of results. I know several thousand things that won't work."

-Thomas A. Edison

The design of the CDF II detector and its trigger system that accommodates heavy flavor physics is conducive towards the search for charm mixing. This analysis used the $D^{0} \rightarrow K \pi$ decay channel data that was available in large quantities in the data collected from February 2002 to January 2009.

\subsection{The Goal of the Analysis}

In order to measure charm mixing, we reconstruct the $D^{*} \rightarrow D^{0} \pi ; D^{0} \rightarrow K \pi$ decay chain and use the flavor tagging method that was briefly discussed in Section 1.3.1. In our analysis, we define right sign (RS) and wrong sign (WS) decays from the relative signs of the pions from $D^{*}$ and from $D^{0}$, as follows:

- Right sign: If pions from $D^{*}$ and $D^{0}$ have the same sign, an event is defined as a right sign decay. For example, $D^{*+} \rightarrow D^{0} \pi^{+} ; D^{0} \rightarrow K^{-} \pi^{+}$. These decays arise from Cabibbo favored decays.

- Wrong sign: If pions from $D^{*}$ and $D^{0}$ have opposite signs, the event is defined as a wrong sign decay. For example, $D^{*+} \rightarrow D^{0} \pi^{+} ; D^{0} \rightarrow K^{+} \pi^{-}$. A wrong sign decay can originate from doubly Cabibbo suppressed decays or through mixing.

- Similarly, we define right sign and wrong sign decays for charge conjugates. For example, $D^{*-} \rightarrow \bar{D}^{0} \pi^{-} ; \bar{D}^{0} \rightarrow K^{+} \pi^{-}$is a right sign decay.

The goal of the analysis is to measure the ratio of wrong sign and right sign decay rates as a function of $D^{0}$ decay time. This ratio, assuming no $\mathrm{CP}$ violation, is given by 


$$
r(t / \tau)=R_{D}+\sqrt{R_{D}} y^{\prime}(t / \tau)+\frac{\left(x^{\prime 2}+y^{\prime 2}\right)}{4}(t / \tau)^{2}
$$

where, as previously defined in Section 1.3.1, $R_{D}=\left|\frac{A(D C S)}{A(C F)}\right|^{2}, x^{\prime}=x \cos \delta+y \sin \delta$ and $y^{\prime}=$ $-x \sin \delta+y \cos \delta, \delta$ is the strong phase difference between DCS and CF amplitudes, $\tau$ is the mean $D^{0}$ lifetime and $t$ is the proper decay time of $D^{0}$.

The mixing parameters $x^{\prime 2}$ and $y^{\prime}$ are determined from the WS/RS ratio. This analysis was performed assuming no $\mathrm{CP}$ violation, and so we combined $D^{*+}$ and $D^{*-}$ data. For a CP-violation study in charm mixing, the same technique of measuring the time dependent WS/RS ratio is employed separately for $D^{*+}$ and $D^{*-}$.

In the following sections, we will discuss the event reconstruction, the data sample that was used for the analysis, the analysis method to extract the number of wrong sign and right sign $D^{*}$ s as a function of decay time, and the final result.

\subsection{Event Reconstruction}

Figure 3.1 illustrates the $D^{*} \rightarrow D^{0} \pi ; D^{0} \rightarrow K \pi$ decay chain in a CDF detector plane transverse to the beam line ( $x-y$ plane). At the primary vertex, a $D^{*}$ meson decays promptly into a pion and a $D^{0}$ meson. The $D^{0}$ meson further decays into $K^{-} \pi^{+}$or $K^{+} \pi^{-}$. The pion from the $D^{*}$ has a lower momentum (and so, a larger curvature) as compared to the pion from the $D^{0}$. This pion is referred to as soft pion throughout this analysis.

The $D^{0}$ candidate is reconstructed from the oppositely charged $K$ and $\pi$ tracks. These tracks are selected using the trigger requirements of the Two-Track Trigger (TTT), which

will be described in the next section. The $D^{0}$ is then combined with the third track of softer momentum to form a $D^{*}$ candidate. This is the tagging pion that is used to determine whether the event is a wrong sign or a right sign event.

The proper decay time of the $D^{0}$ is measured using the transverse decay length $L_{x y}$, given by

$$
L_{x y}=\frac{\mathbf{r} \cdot \mathbf{p}_{\mathbf{T}}}{\left|\mathbf{p}_{\mathbf{T}}\right|}
$$




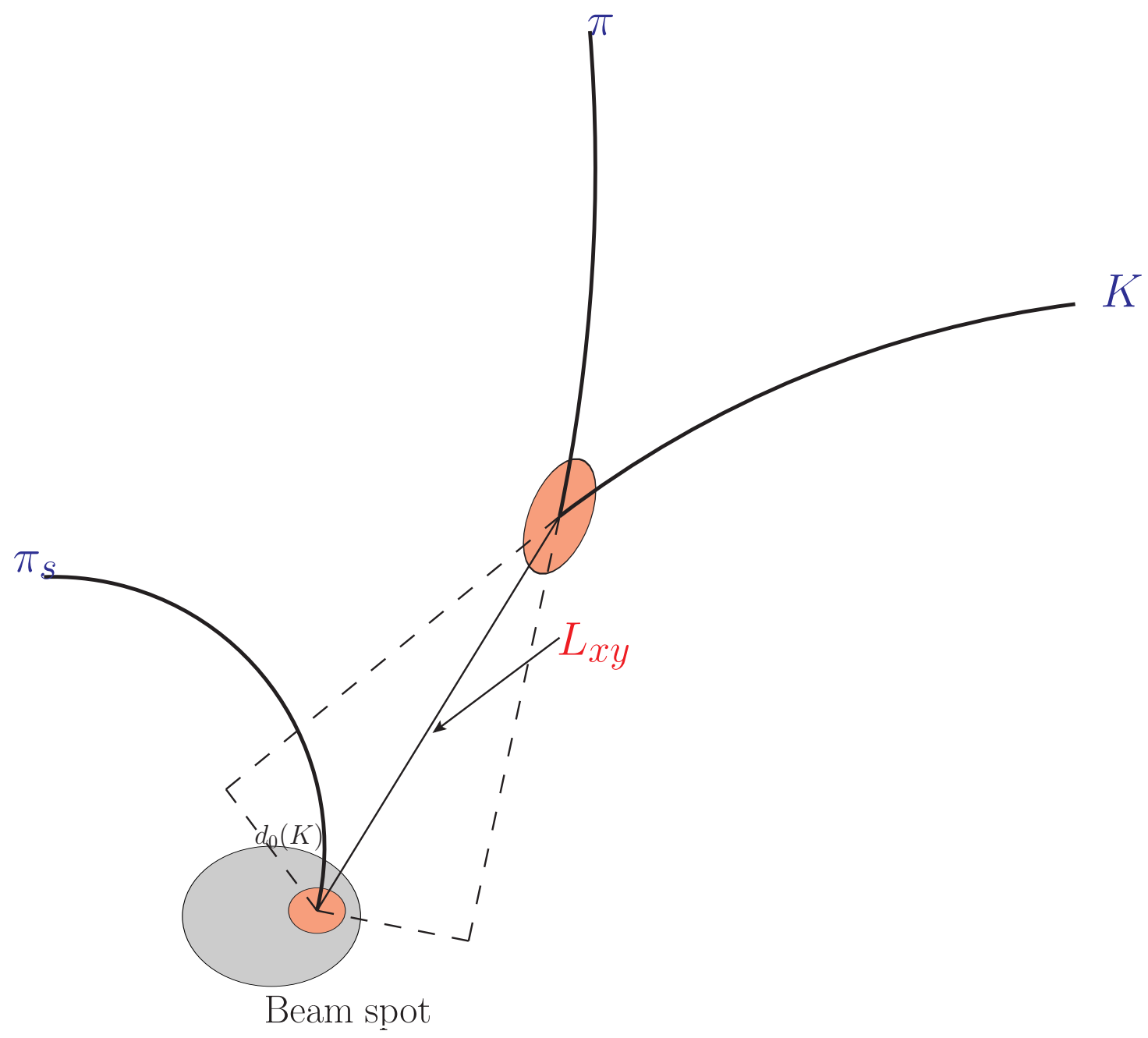

Figure 3.1. Illustration of $D^{*} \rightarrow D^{0} \pi ; D^{0} \rightarrow K \pi$ decay chain at the CDF II detector in the $x-y$ plane transverse to beam line. The $D^{*}$ decays promptly into $\pi$ and $D^{0}$ and the $D^{0}$ further decays into $K$ and $\pi$. The figure also illustrates decay length $\left(L_{x y}\right)$ of $D^{0}$. The impact parameter $d_{0}$ for a kaon track is illustrated as an example.

where $\mathbf{r}$ is the vector pointing to the $D^{0}$ decay vertex, and $\mathbf{p}_{\mathbf{T}}$ is the transverse momentum of the $D^{0}$. The proper decay time $t$ is calculated from the equation

$$
c t=\frac{L}{\gamma \beta}=\frac{c L_{x y} M_{D^{0}}}{\left|\mathbf{p}_{\mathbf{T}}\right|},
$$

where $L$ is the decay length of the $D^{0}$ meson, $\beta=v / c, \gamma=1 / \sqrt{1-\beta^{2}}$, and $M_{D^{0}}$ is the mass of the $D^{0}$ meson.

Since $L_{x y}$ is measured from the primary vertex, we can accurately measure the proper 
decay time by requiring that the $D^{*}$ comes from the primary vertex.

Figure 3.1 also illustrates another important variable - the impact parameter of a track. The impact parameter of a track is defined as the distance of closest approach from the primary vertex to the extrapolated trajectory of the track, in the plane transverse to the beam line. In the Figure 3.1, the impact parameter of a kaon track is shown as $d_{0}(K)$. The impact parameter can also be computed for other tracks. The formula to compute the impact parameter is

$$
d_{0}=\frac{\hat{\mathbf{z}} \cdot\left(\mathbf{r} \times \mathbf{p}_{\mathbf{T}}\right)}{\left|\mathbf{p}_{\mathbf{T}}\right|}
$$

where $\hat{\mathbf{z}}$ is the unit vector along the $z$-axis.

The impact parameter distribution plays an important role in the analysis to distinguish prompt $D^{*}$ s originating at the primary vertex from the non-prompt $D^{*}$ s originating at the secondary vertices. This is explained in detail in Section 3.6.4.

\subsection{Two Track Trigger}

The events are selected in real-time using the two-track trigger (TTT). The TTT was developed for a broad range of heavy flavor physics, especially b-Physics (physics of hadrons involving $b$ quarks, such as $B$ mesons). The trigger has a good acceptance for charm mesons due to some similarities between $B$ and charm mesons. Both $B$ and charm mesons have longer lifetimes as compared to the random combinatorial background, and smaller lifetimes compared to strange particles. The trigger, therefore, looks for a displaced vertex (to avoid background from the primary interaction), with characteristics consistent with the decay of long lived particles.

Along with good a charm acceptance, the trigger also has good time resolution of greater than $0.16 \times D^{0}$ lifetime. We confirm that both $K$ and $\pi$ tracks are the trigger tracks, satisfying the TTT trigger requirements.

In the TTT trigger, at Level 1, the extremely fast tracker (XFT) reconstructs COT tracks. The Level 1 requirements defined for the XFT are listed below:

- The trigger requires two oppositely charged tracks. 
- The XFT reconstructs these tracks from four COT axial superlayers.

- The transverse momentum $p_{T}$ of each of the individual tracks is greater than 2.04 $\mathrm{GeV} / c$

- The scalar sum of the $p_{T} \mathrm{~s}$ of tracks is greater than $5.5 \mathrm{GeV} / c$.

- The angle between the two tracks should be between 0 and 135 degrees.

At Level 2, all Level 1 constraints are applicable, with some requirements made more stringent and new constraints added. The displaced vertex is identified by the secondary vertex tracker (SVT) at Level 2. The Level 2 requirements are:

- All Level 1 requirements satisfied.

- Impact parameter $d_{0}$ of each track is between $0.12 \mathrm{~mm}$ and $1.0 \mathrm{~mm}$.

- The angle between two tracks is now required to lie between 0 and 90 degrees.

- The $\chi^{2}$ of the SVT fit is required to be less than 25 .

- The intersection point in the $r-\phi$ plane projected along the net momentum vector of the two tracks is required to be greater than $200 \mu \mathrm{m}$ from the beam line.

At Level 3, the COT tracks are extrapolated and matched with SVT tracks. Once the matches are made, constraints similar to Level 1 and Level 2 requirements are applied.

The triggers are prescaled to handle high data rates. The prescaling of the TTT lowers the $p_{T}$ cuts when the luminosity falls below a certain magnitude. This prescaling does not affect our analysis, since it has the same effect on RS and WS decays.

The events passing the above trigger requirements are written to tapes in the mass storage system for offline processing.

\subsection{Data Sample}

The data used for this analysis was collected from February 2002 to January 2009. This data corresponds to an integrated luminosity of $\approx 4.0 \mathrm{fb}^{-1}$ for $p \bar{p}$ collisions at a center of mass energy $\sqrt{s}=1.96 \mathrm{TeV}$. 
The data is extracted from the data files prepared for $B$ physics. The luminosity was determined by selecting the "good" runs and the following detector components in the run summary data base: Level 1 to 3 triggers, CLC, COT, CMU, CMP, CMX, SVX, ISL, and Layer 00 of silicon.

\subsection{A Brief History of This Analysis}

The measurement of the ratio of branching fractions of DCS to CF decays (time independent) was performed with $0.35 \mathrm{fb}^{-1}$ of integrated luminosity. This result was published in 2006 [43]. It was during this study that the method to extract $D^{*}$ yields was developed and most of the cuts were optimized using the $D^{*}$ yield technique.

The time dependent measurement of the ratio of DCS and CF decay rates was performed with $1.5 \mathrm{fb}^{-1}$ integrated luminosity using the same $D^{*}$ yield technique. The data was collected from February 2002 to January 2007. This measurement provided the evidence for $D^{0}-\overline{D^{0}}$ mixing in $2007[22]$.

We extended the analysis technique by exploring new cuts and by applying an Artificial Neural Network. These methods are described in detail in Appendix A. However, no significant improvements were observed by using the new cuts. The Artificial Neural Network analysis yielded similar results to the standard analysis technique that was employed in the previous publications. So no changes were proposed to the existing technique and the same technique was employed for the current analysis with about $4.0 \mathrm{fb}^{-1}$ of integrated luminosity.

\subsection{Analysis}

\subsubsection{Backgrounds and Basic Cuts}

While looking for the wrong sign signal, we encounter four sources of backgrounds: misidentification of $K K$ and $\pi \pi$ tracks as $K \pi$ tracks, mis-identification of $\pi K$ track as $K \pi$ track, random pion background, and combinatorial background. These backgrounds are discussed below. The signal and background shapes are illustrated in Figure 3.2. The $D^{*}$ mass difference illustrated in this sketch is defined as $\Delta M=\mathrm{m}\left(D^{*}\right)-\mathrm{m}\left(D^{0}\right)-\mathrm{m}\left(\pi_{s}\right)$. 


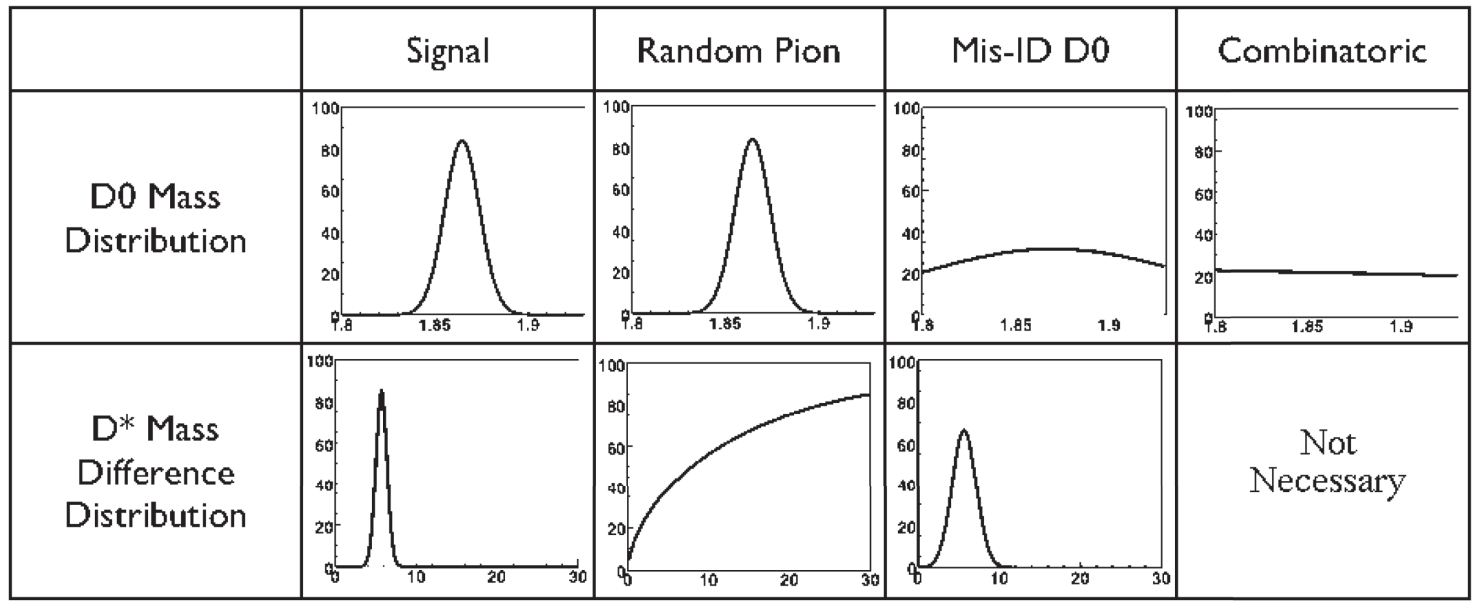

Figure 3.2. A sketch to illustrate signal and backgrounds in $K \pi$ mass and mass difference distributions. The $D^{0}(K \pi)$ mass distribution has the range from 1.80 to $1.92 \mathrm{GeV} / c^{2}$ on the $x$-axis and the number of events per $2 \mathrm{MeV} / c^{2}$ on the $y$-axis. The mass difference distribution has the range from 0 to $30 \mathrm{MeV} / c^{2}$ on the $x$-axis and the number of events per $0.5 \mathrm{MeV} / c^{2}$ on the $y$-axis [44].

\section{Mis-identification backgrounds}

We reconstruct a $D^{0}$ candidate from two oppositely charged tracks with both $K \pi$ and $\pi K$ interpretations. When we combine the reconstructed $D^{0}$ with a third track for the soft pion, we get a $D^{*}$ candidate with wrong sign and right sign $D^{0}$ s. A correct mass assignment will result in a narrow peak in the mass distribution plot.

Figure 3.3 shows the mass distribution for wrong sign (along $x$-axis) and right sign (along $y$-axis ) interpretations. The plot has events clustered in the high mass (upper right) and low mass (lower left) regions. These clusters are $\pi^{+} \pi^{-}$and $K^{+} K^{-}$events reconstructed as $K \pi$ events. To remove this background, we restrict the $K \pi$ mass region to $1.8 \leq \mathrm{m}_{K \pi} \leq 1.92$ $\mathrm{GeV} / c^{2}$. The resulting plot is shown in Figure 3.4.

In Figure 3.4, we notice that the wrong sign signal (vertical yellow band) is dominated by the broad right sign distribution (horizontal red band). This happens because the kaon and the pion assignments of the $D^{0}$ daughter tracks are interchanged. To reduce this particular background, we apply a mass cut on right sign events corresponding to a 40 $\mathrm{MeV} / c^{2}$ window around the world average $D^{0}$ mass as depicted in Figure 3.5. This cut is defined as $\left|m_{K \pi}(R S)-m_{D^{0}}\right| \leq 20 \mathrm{MeV} / c^{2}$. We call this requirement the "opposite assignment mass cut". This removes most of the red colored background. 


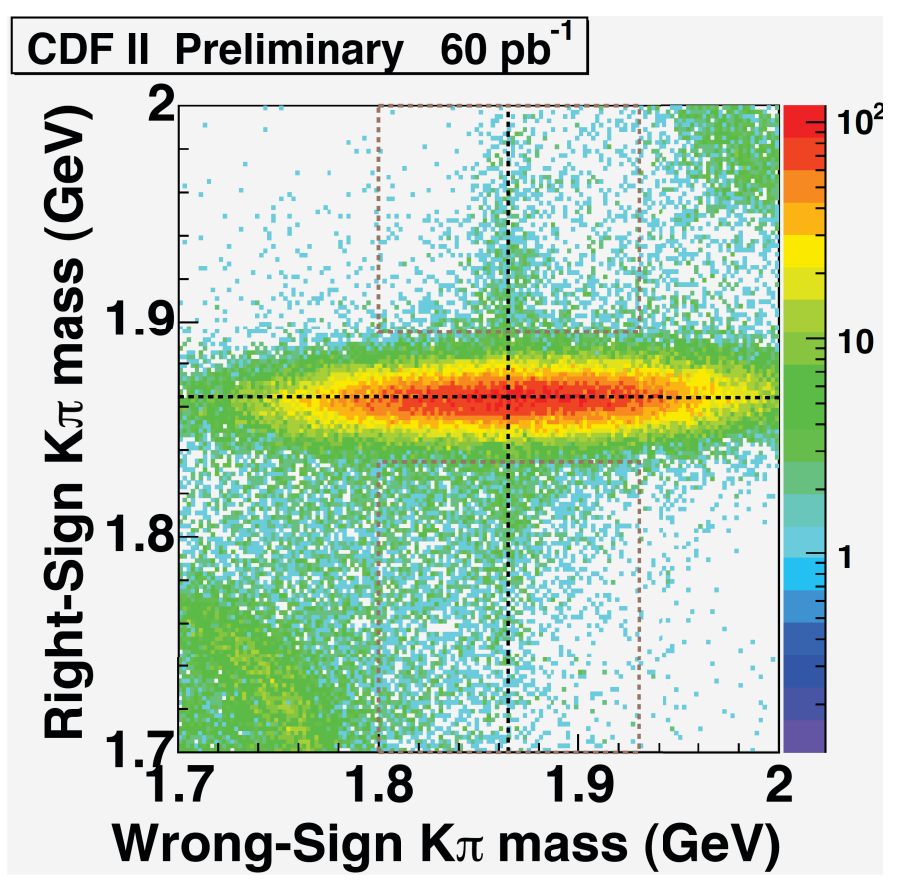

Figure 3.3. WS and RS $K \pi$ mass plotted together (using $60 \mathrm{pb}^{-1}$ data). The dashed lines correspond to the world average $D^{0}$ mass, $1.8645 \mathrm{GeV} / c^{2}[45]$.

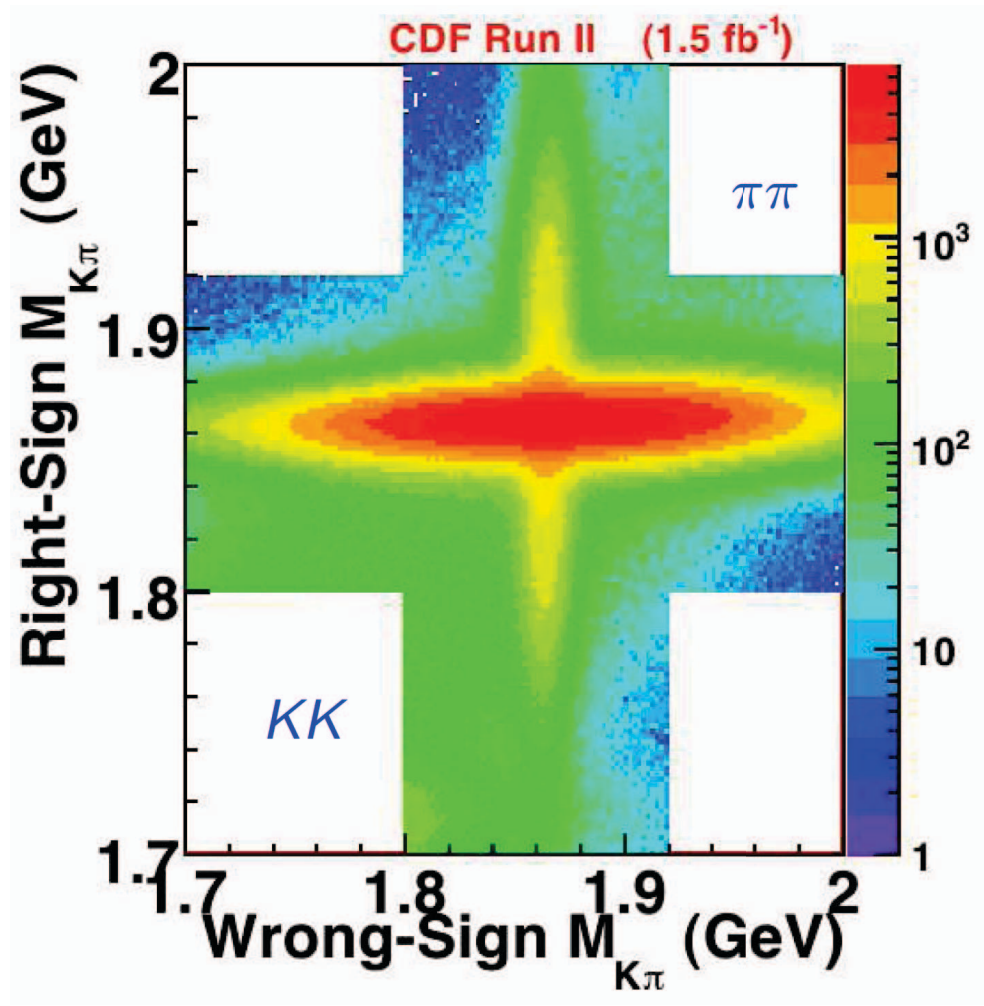

Figure 3.4. WS and RS plot after removing $K K$ and $\pi \pi$ background (using $1.5 \mathrm{fb}^{-1}$ data) [44]. 


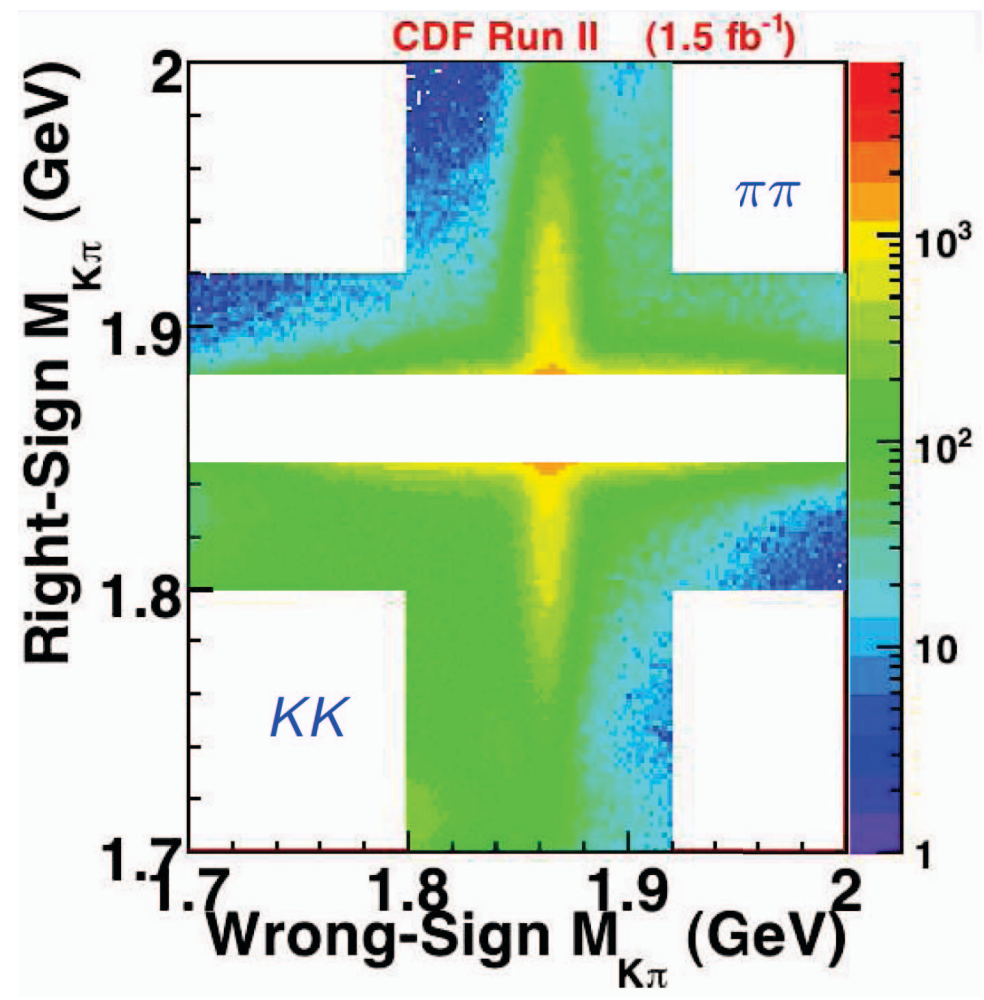

Figure 3.5. Opposite assignment mass cut on RS $K \pi$ mass for WS signal using $1.5 \mathrm{fb}^{-1}$ data) [44].

To further clean up the right sign background from wrong sign signal, we apply a particle identification cut. The particle identification cut uses ionization energy loss per unit length $(\mathrm{dE} / \mathrm{dx})$ measured in the multi-wire drift chamber $(\mathrm{COT})$. In our analysis, we use a $Z$ variable defined as

$$
Z=\log \left(\frac{\frac{\mathrm{dE}}{\mathrm{dx} \text { measured }}}{\frac{\mathrm{dE}}{\mathrm{dx} \text { expected }}}\right)
$$

For correctly identified $K$ tracks, the $Z$ distribution will be a unit Gaussian centered at $Z=-0.44$. For correctly identified $\pi$ tracks, the $Z$ distribution will be centered at $Z=$ -0.51. Real kaon tracks incorrectly interpreted as pion tracks (pion hypothesis) will have a $Z$ Gaussian distribution centered at -1.6 . For a kaon hypothesis, a real pion will be centered at 0.6 .

Figure 3.6 shows the $Z$ distributions for the kaon track with both $K$ and $\pi$ hypotheses. Figure 3.7 shows the same for a pion track.

The idea behind the particle identification cut is to compare the probability distributions 
for $K \pi$ and $\pi K$ assignments and to use the more probable hypothesis. For the RS decay, $D^{0} \rightarrow K^{-} \pi^{+}$, with RS hypothesis, the $Z$ value of the first track is $Z_{K, 1}$, and the $Z$ value for the second track is $Z_{\pi, 2}$. With $K \pi$ assignments interchanged, the $Z$ values of the first and the second track will be $Z_{\pi, 1}$ and $Z_{K, 2}$, respectively.

The cut variable is then defined as

$$
\left(Z_{K, 1}^{2}+Z_{\pi, 2}^{2}\right)-\left(Z_{\pi, 1}^{2}+Z_{K, 2}^{2}\right)
$$

for which the RS $D^{0}$ will have a negative value, since the WS sum of squares will be larger than the RS sum of squares. Similarly, for the WS $D^{0}$, the cut variable will have a positive value.

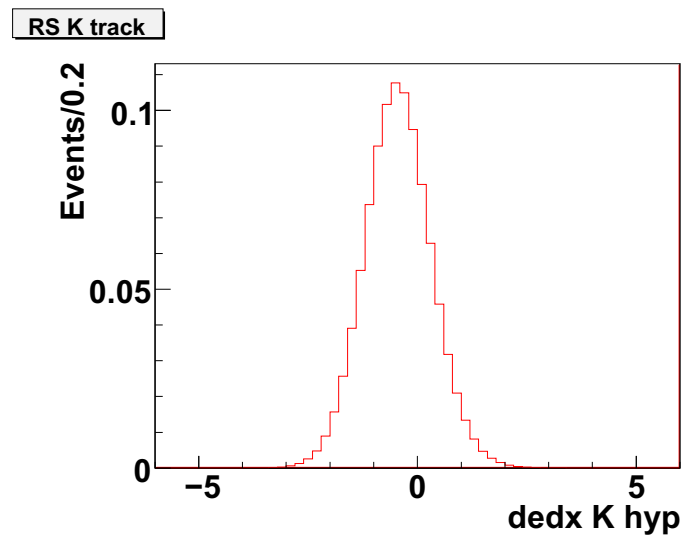

(a)

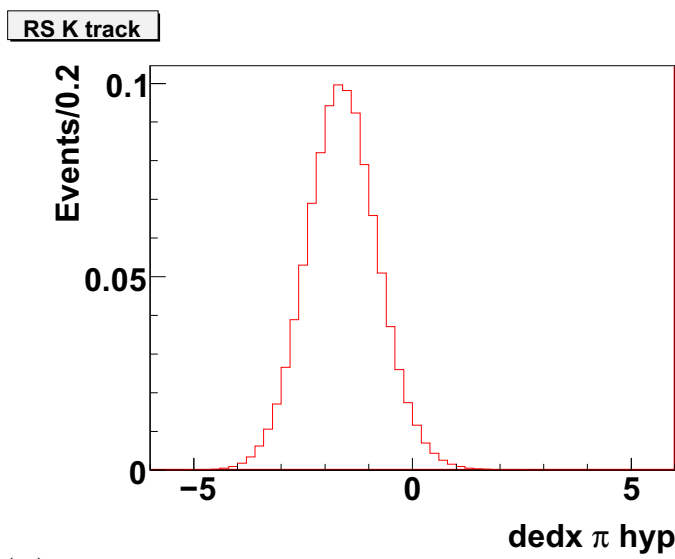

(b)

Figure 3.6. $\mathrm{dE} / \mathrm{dx}$ ( $Z$ variable) distribution for a kaon track (a) with a kaon hypothesis and (b) with a pion hypothesis.

The two cuts, opposite mass assignment cut and particle identification cut, together remove more than $96.4 \%$ of the right sign background from wrong sign signal and retain $78 \%$ of the signal. Figure 3.8 depicts RS and WS distributions before and after the two cuts are applied.

\section{Random Pion Background}

We call the background generated from combining a $D^{0}$ with a random pion track not associated with a $D^{0}$ the random pion background. 


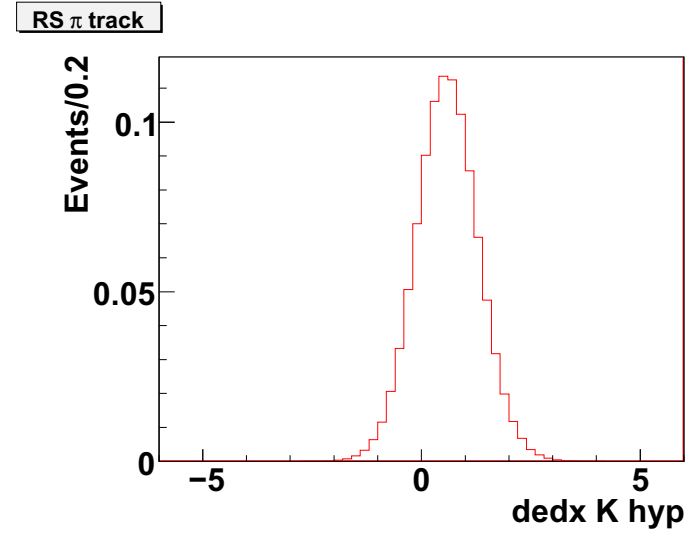

(a)

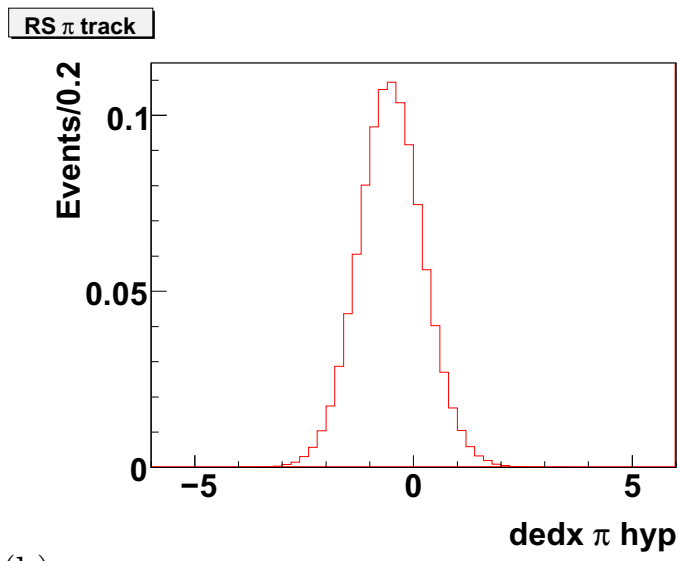

(b)

Figure 3.7. $\mathrm{dE} / \mathrm{dx}(Z$ variable) distribution for a pion track (a) with a kaon hypothesis and (b) with a pion hypothesis.

\section{Combinatorial Background}

In some events, one or both tracks do not belong to a $D^{0} \rightarrow K \pi$ decay. The candidates formed from such a random selection of tracks sometimes happen to pass our selection criteria. These events form the combinatorial background.

Figure 3.9 is the projection of WS $D^{0}$ fit results obtained from $0.35 \mathrm{fb}^{-1}$ data. This figure illustrates the relative amounts of signal and backgrounds. The combinatorial background can be modelled as a linear or quadratic decreasing function.

To reduce the combinatorial background, we apply a series of cuts. We require the transverse decay length significance $L_{x y} / \sigma_{x y}$ to be greater than 4 , where $\sigma_{x y}$ is the uncertainty on $L_{x y}$. The impact parameter for the soft pion track must have $d_{0}<500 \mu \mathrm{m}$. The soft pion must also have a point of closest approach to the primary vertex less than $1.5 \mathrm{~cm}$ along the beam line, and transverse momentum greater than $0.3 \mathrm{GeV} / c$.

\section{Cut Optimization}

All the cuts were optimized in the time-independent study performed with $0.35 \mathrm{fb}^{-1}$ integrated luminosity and using the time integrated $D^{*}$ yield technique for RS events [43]. The cuts were chosen to maximize the signal significance $\frac{\mathrm{S}}{\sqrt{\mathrm{S}+\mathrm{B}}}$, where $S$ is the WS signal and $B$ is the WS background. The optimization was performed with the WS signal kept blinded. The WS signal was estimated by scaling the RS signal by the measured time integrated 

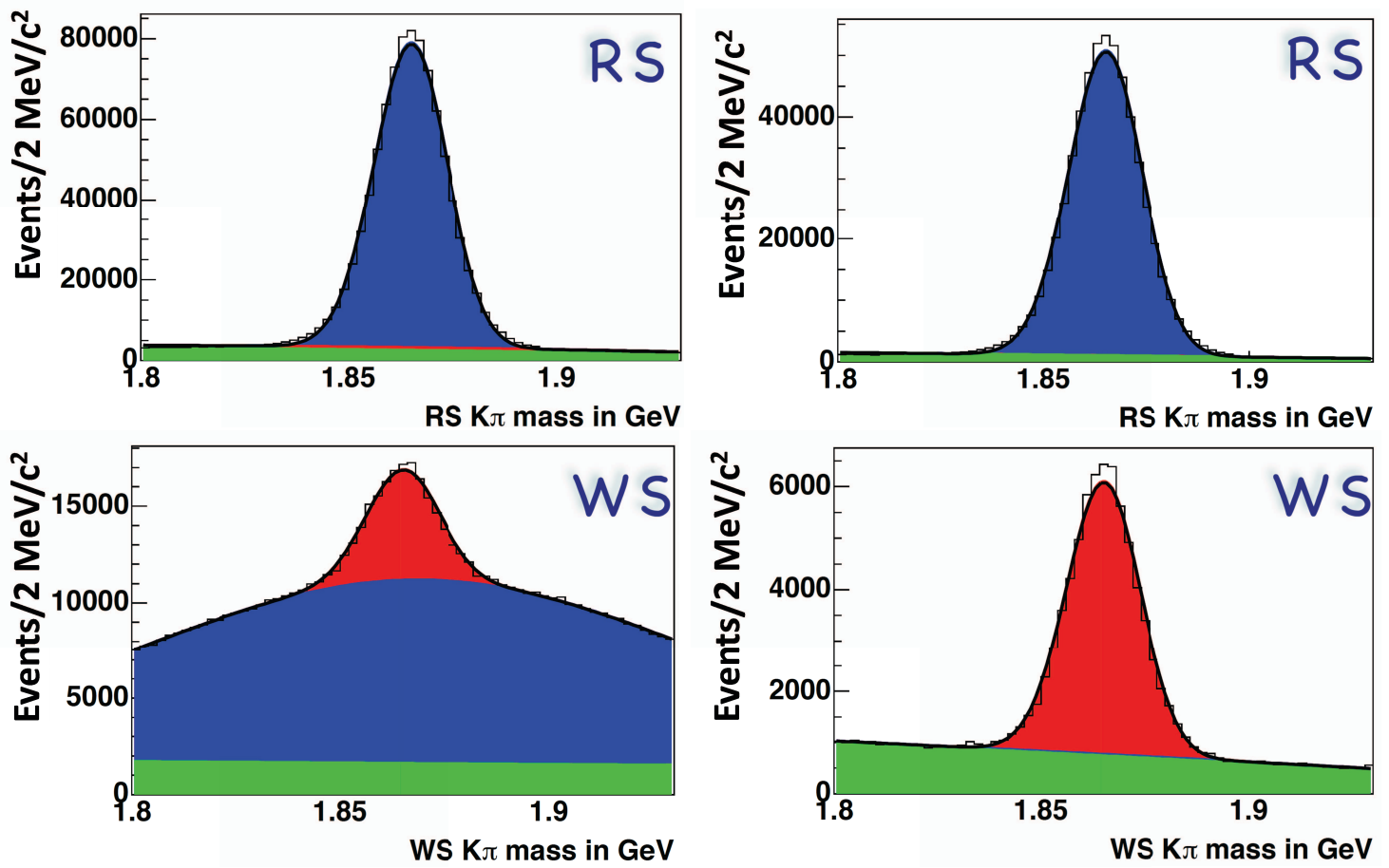

Figure 3.8. The right sign and wrong sign distributions with $0.35 \mathrm{fb}^{-1}$ data. The figures on the left are right sign and wrong distributions before the opposite assignment mass cut and PID cuts are applied. The figures on the right are the RS and WS distributions after the application of these cuts. In all these plots, the red colored peak region is the RS(WS) signal. The blue colored broad background is mis-identified background. The green colored linear distribution is from the combinatorial background [44].

ratio of WS/RS rates $R_{D}=0.0035$. The WS background was estimated from the region outside the signal peak in the $D^{*}$ mass plot [45].

\subsubsection{The Clean RS Signal}

Figure 3.10 shows the fits for the time integrated RS $K \pi$ mass plot using the data with $\left|d_{0}\right|<60 \mu \mathrm{m}$. The plot shows a huge and pure RS $D^{0}$ signal, shown by a dark gray colored fit projection and a small background at the bottom shown by a light gray fit. The fits are described in the next section.

The right sign and wrong sign decays have the same kinematics, so they will have the same distributions. Due to this fact, we can obtain probability distribution shapes from right sign decays and use them for the wrong sign decays. This technique eliminates the need for Monte Carlo simulations and avoids the systematic uncertainties arising from fixing 


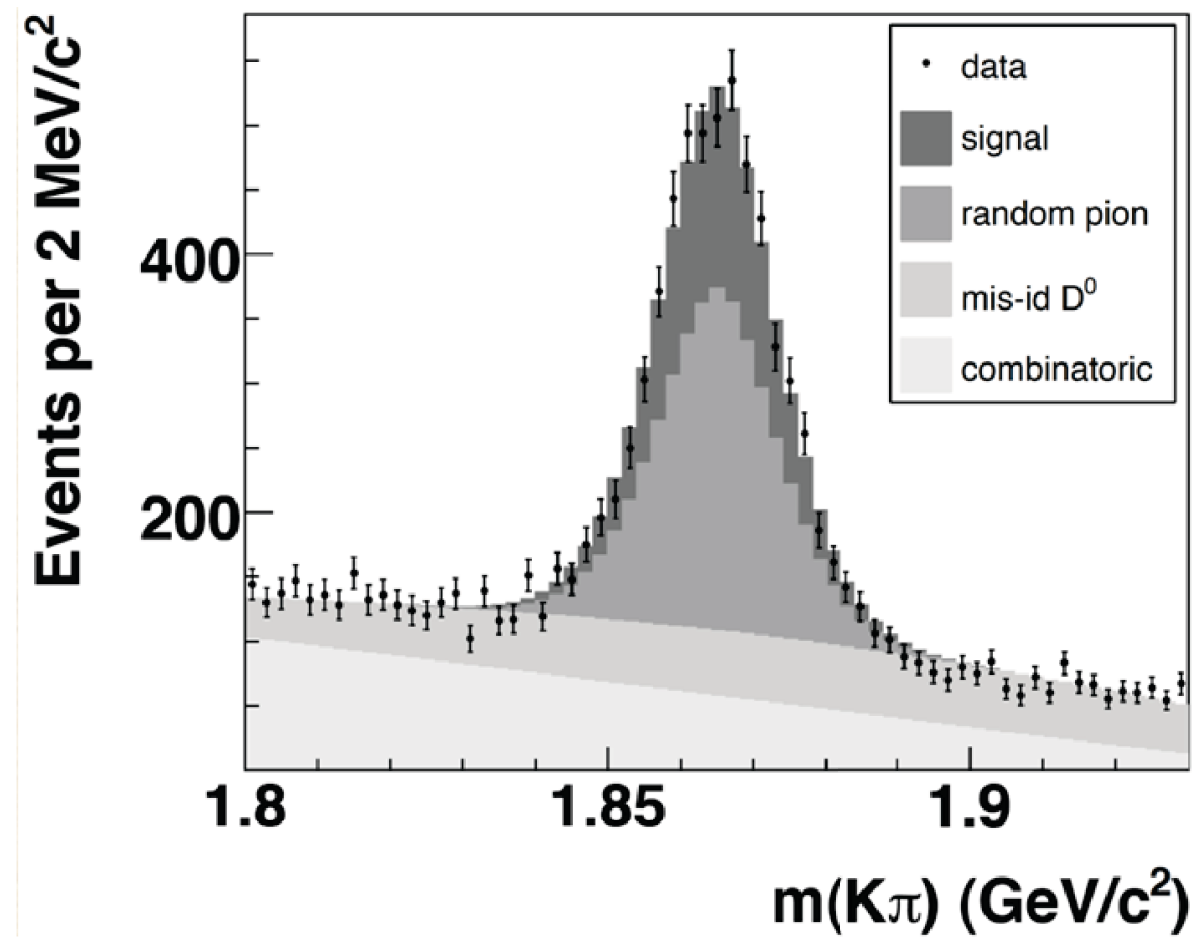

Figure 3.9. Projection of WS $D^{0}$ fit results obtained from $0.35 \mathrm{fb}^{-1}$ data to illustrate relative amounts of signal and backgrounds [43].

the shapes from Monte Carlo.

Another advantage of this technique is that, in measuring the WS/RS ratio, most of the systematic uncertainties are cancelled in the ratio. This will be described in Section 3.7.2.

\subsubsection{The $D^{*}$ Yield Technique}

\section{$K \pi$ Mass Fits}

In order to extract RS and WS $D^{*}$ s, the first step in our analysis is to perform $K \pi$ mass fits to extract the number of $D^{0}$ s. We adopted the following approach:

1. Divide the data into RS and WS events.

2. Divide each of the RS and WS data into 20 time bins.

3. Divide each time bin into 60 mass difference $(\Delta M)$ bins.

4. For a given time bin and a given mass difference bin, fit the $K \pi$ mass distribution.

The choice of time bins is based on statistics. The following choices were made: 


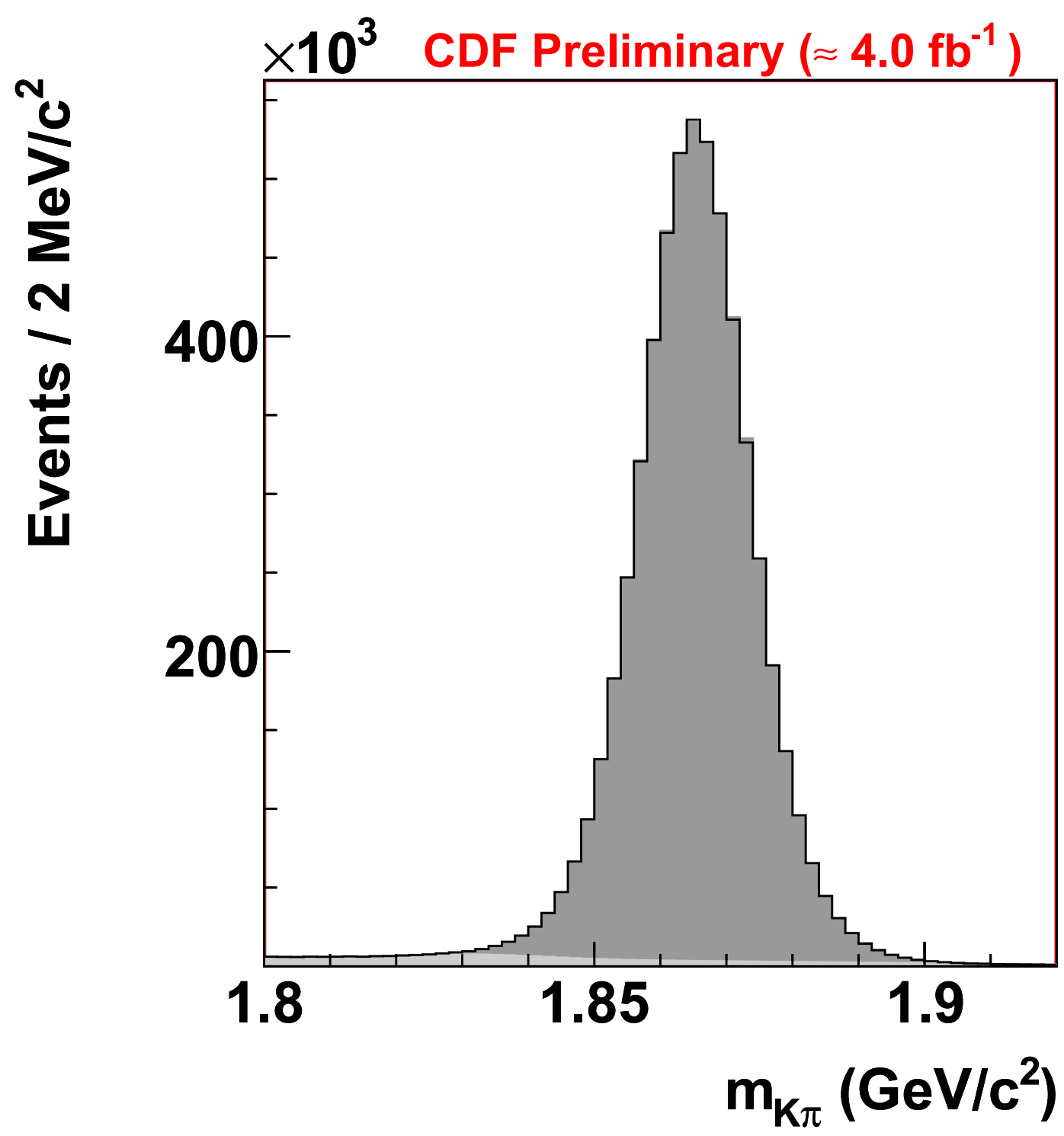

Figure 3.10. The time integrated RS $D^{0}$ sample. Dark grey is the signal fit, light grey is the background fit.

- 13 time bins of width $0.25 D^{0}$ lifetime, for decay times from $0.75-4.0 D^{0}$ lifetimes.

- 4 time bins of width $0.5 D^{0}$ lifetime, for decay times from 4.0 - $6.0 D^{0}$ lifetimes.

- 2 time bins of width one $D^{0}$ lifetime, for decay times from $6.0-8.0 D^{0}$ lifetimes.

- 1 time bin of width $2.0 D^{0}$ lifetime, for decay times from 8.0 - $10.0 D^{0}$ lifetimes. 
Time slice $2.75<\mathrm{t} / \tau<3.00$

$5.5<\Delta \mathrm{M}<6.0 \mathrm{MeV} / \mathrm{c}^{2}$

CDF Run II $\left(\approx 4.0 \mathrm{fb}^{-1}\right.$

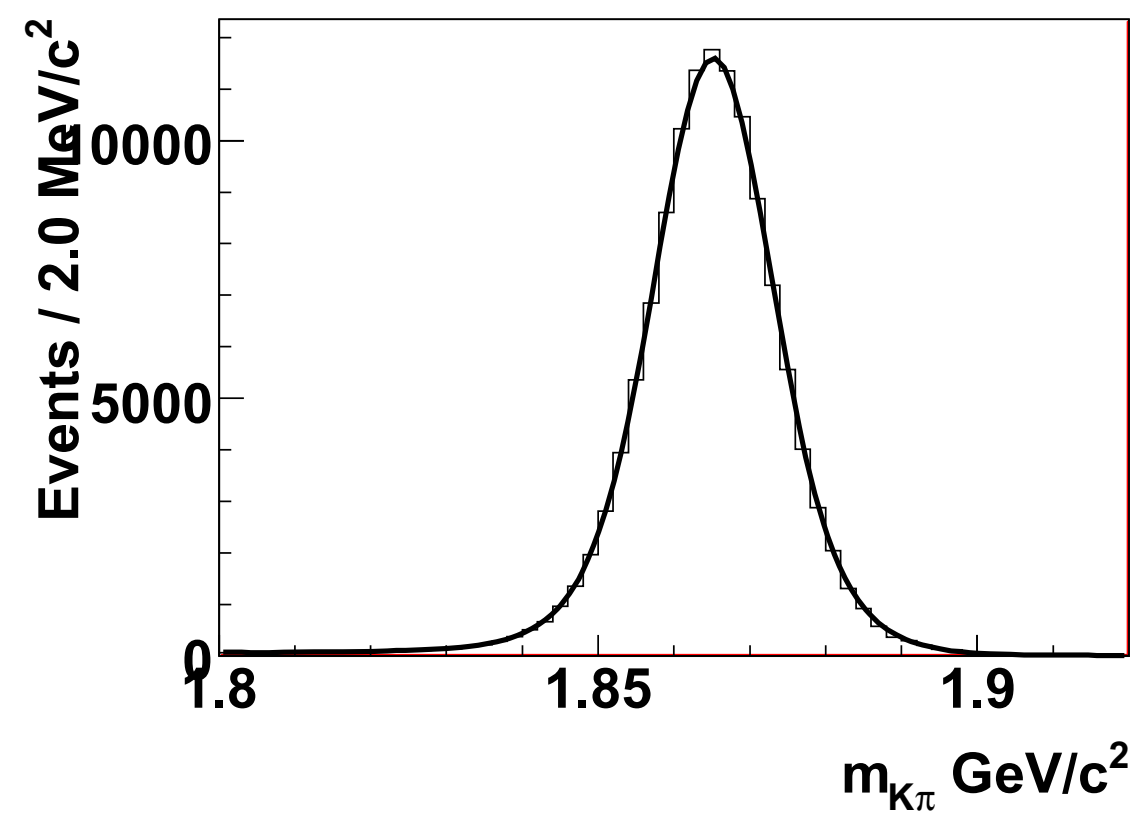

Figure 3.11. RS $K \pi$ mass fit for $2.75<t / \tau<3.00$ and $5.5<\Delta M<6.0 \mathrm{MeV} / c^{2}$.

Since we divide the mass difference range $0<\Delta M<30 \mathrm{MeV} / c^{2}$ into 60 bins, the size of each bin is $0.5 \mathrm{MeV} / c^{2}$.

Figure 3.11 shows an example of the RS $K \pi$ mass fit for the time bin $2.75<t / \tau<3.00$ and mass difference bin $5.5<\Delta M<6.0 \mathrm{MeV} / c^{2}$. Figure 3.12 shows the WS $K \pi$ mass fit for the same time and mass difference bins.

The signal shape is a double Gaussian. The mean of the first Gaussian is $1.8652 \pm 0.00001$ $\mathrm{GeV} / c^{2}$ and that of the second Gaussian is $1.8648 \pm 0.00003 \mathrm{GeV} / c^{2}$. The widths of the two Gaussians are 7.19 and $11.63 \mathrm{MeV} / c^{2}$, respectively. The signal shapes for $K \pi$ mass distributions are fixed from the time integrated $\mathrm{RS} K \pi$ mass distribution that was shown in Figure 3.10. The same shapes are used for both RS and WS distributions. For these fits, a typical $\chi^{2} /$ ndf $\approx 1.0$, where ndf stands for number of degrees of freedom.

For each $m_{K \pi}$ distribution, a parabola with floating parameters is used to fit the background. The amplitudes of the signal and background shapes are determined independently for all $K \pi$ distributions. 
Time slice $2.75<\mathrm{t} / \tau<3.00$

$5.5<\Delta \mathrm{M}<6.0 \mathrm{MeV} / \mathrm{c}^{2}$

CDF Run II $\left(\approx 4.0 \mathrm{fb}^{-1}\right.$

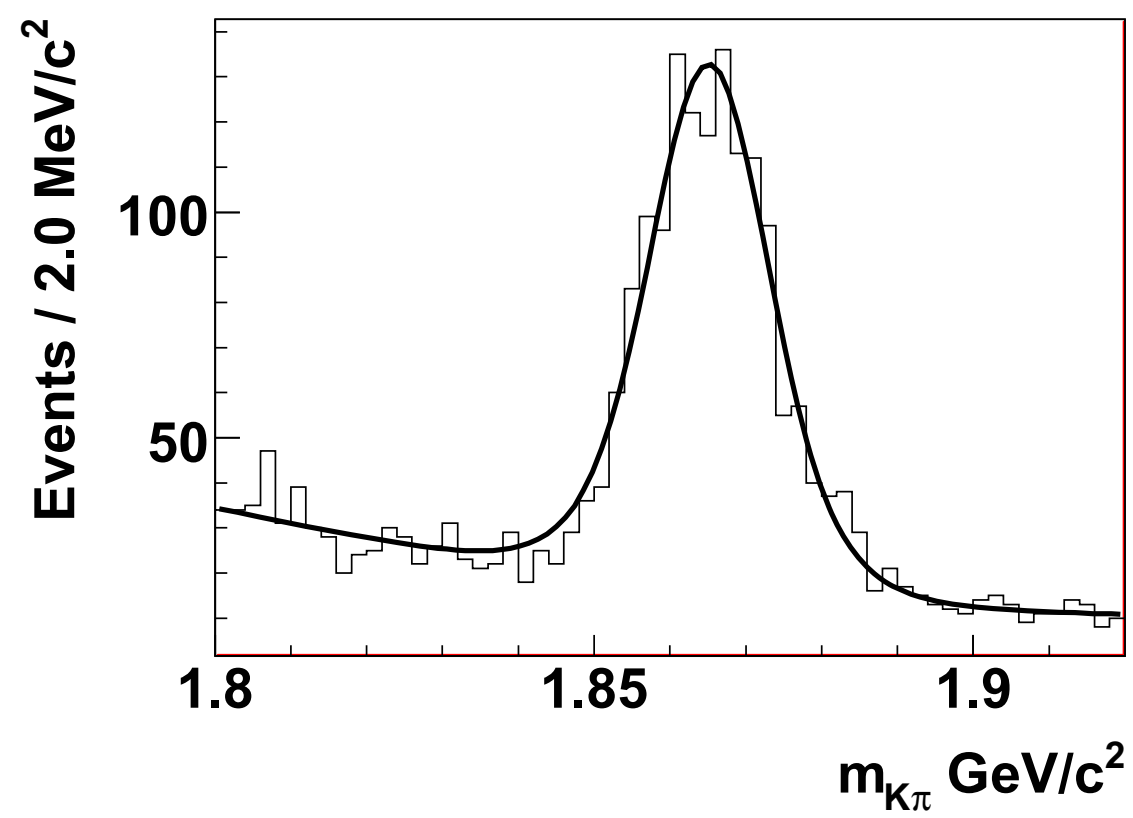

Figure 3.12. WS $K \pi$ mass fit for $2.75<t / \tau<3.00$ and $5.5<\Delta M<6.0 \mathrm{MeV} / c^{2}$.

The signal peak has correctly reconstructed $D^{0}$ candidates with some background from random pions. The background contains mis-identification and combinatorial backgrounds.

The $K \pi$ mass fit gives the $D^{0}$ yield for a given time bin and mass difference bin.

\section{Mass Difference Fits}

For a given time bin, when we fit the $K \pi$ mass distributions for all 60 mass difference bins, we get a number of $D^{0}$ vs. mass difference distributions. On fitting these distributions, we get the $D^{*}$ yield. Figures 3.13 and 3.14 show the RS and WS $\Delta M$ fits for time bin $2.75<t / \tau<3.00$.

As in the $K \pi$ mass fits, the signal shapes in all time dependent $\Delta M$ distributions were fixed from the time integrated right sign $\Delta M$ distribution. The same shapes were applied to both the RS and WS $\Delta M$ distributions.

The background shape has a power term of $\Delta M$. The background shapes for all the RS $\Delta M$ distributions were fixed from the time integrated RS $\Delta M$ distributions. Similarly, the 


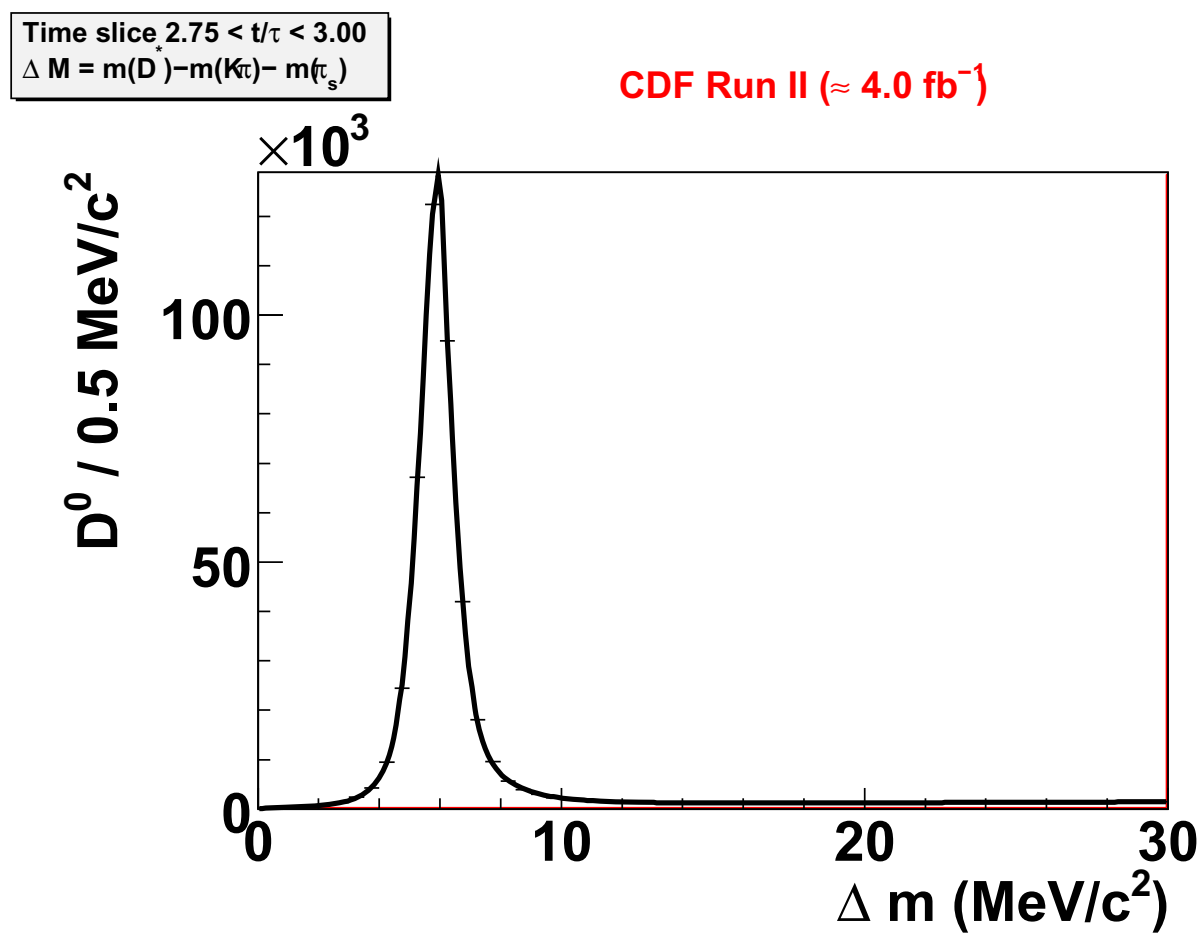

Figure 3.13. RS $D^{*}$ distribution for $2.75<t / \tau<3.00$.

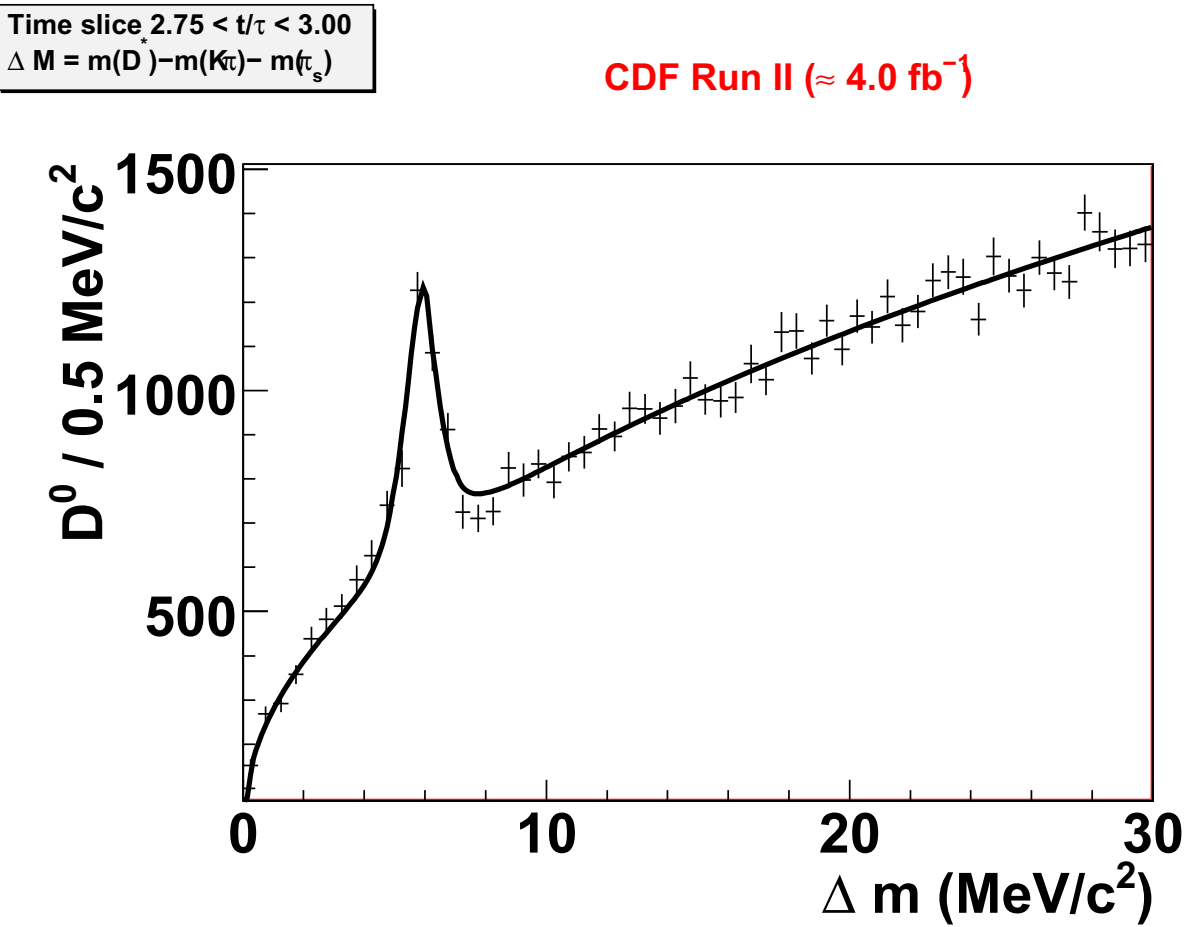

Figure 3.14. WS $D^{*}$ distribution for $2.75<t / \tau<3.00$. 
background shapes for all the WS $\Delta M$ distributions were fixed from the time integrated WS $\Delta M$ distributions. The amplitudes of the signal and background shapes are determined independently for all $\Delta M$ distributions.

The signal peak has the correctly reconstructed $D^{*}$ s. The background is from correctly reconstructed $D^{0}$ s combined with a random pion not associated with the $D^{0}$.

The RS and WS distributions have approximately the same amount of background. However, the RS signal is about 250 times larger than the WS signal.

The $\Delta M$ distribution fits give the $D^{*}$ yield for a given time bin. We do this for all 20 time bins for both RS and WS events to get the number of RS and WS $D^{*}$ s in each of the time bins. The time integrated RS and WS $\Delta M$ distributions are shown in Figures 3.15 and 3.16 .

For $4.0 \mathrm{fb}^{-1}$ integrated luminosity, the time integrated $\mathrm{RS} D^{*}$ yield was $\approx 5.7$ million. The time integrated WS $D^{*}$ yield was 24000 .

We can use this yield to estimate the expected mixing significance as a "back of the envelope" calculation. The time integrated RS $D^{*}$ yield for the result published in 2007 with $1.5 \mathrm{fb}^{-1}$ data was 3.0 million. The published result provided the mixing significance of $\sigma_{\text {old }}=3.8$. If we assume that the central values of the mixing parameters remain the same and that the significance is proportional to the square root of the number of events (significance $\propto \sqrt{N}$ ), then we can estimate the expected significance $\left(\sigma_{\text {exp }}\right)$ using:

$$
\sigma_{\text {exp }}=\sigma_{\text {old }} \times \sqrt{\frac{N_{\text {total }}}{N_{\text {old }}}}
$$

With $\sigma_{\text {old }}=3.8, N_{\text {total }}=5.7$ million, $N_{\text {old }}=3.0$ million, we get $\sigma_{\text {exp }}=5.23$.

\subsubsection{Non-prompt $D^{*}$ Correction}

Before we look for the ratio of WS and RS $D^{*}$ s as a function of $D^{0}$ decay time, we have one more step to complete. Since we measure the proper decay time of the $D^{0}$ from its transverse decay length $L_{x y}$, and as $L_{x y}$ is measured from the primary vertex, we need to ensure that the $D^{*}$ s originate at the primary vertex (called prompt $D^{*}$ s). This also avoids the complication of measuring the proper decay time of a $D^{0}$ from a secondary vertex (non- 


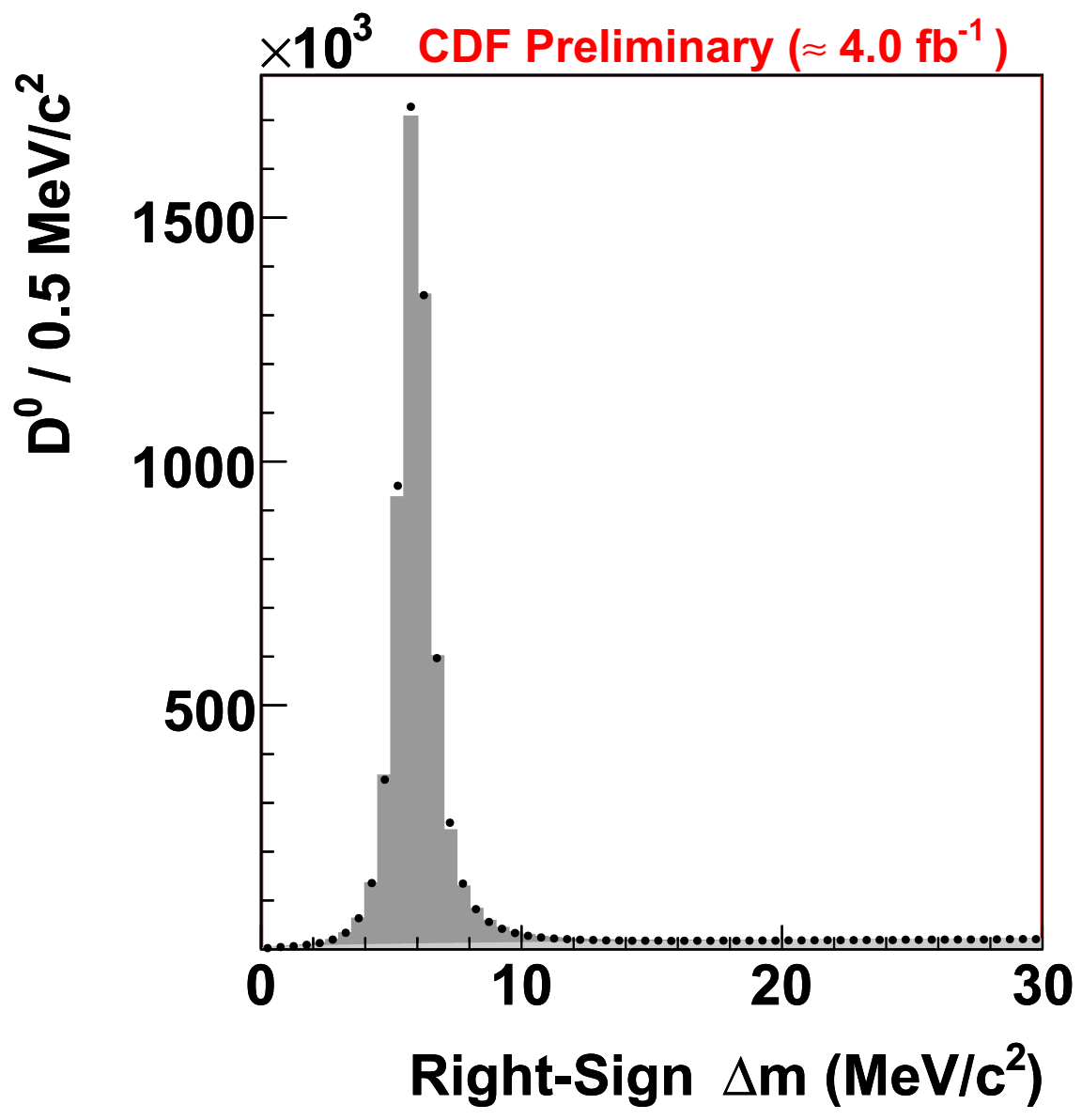

Figure 3.15. Time integrated right sign $\Delta M$ distribution.

prompt $D^{*}$ s). For example, the $D^{*}$ originating at a secondary vertex from a $B$-decay will have a wrong decay time.

We use the impact parameter (IP) distribution of $D^{0}$ to eliminate the non-prompt $D^{*}$ s. The $D^{0}$ mesons originating from the prompt $D^{*}$ s have a narrow impact parameter $\left(d_{0}\right)$ distribution. The $D^{0}$ mesons produced from the $D^{*}$ mesons coming from secondary decays have a wide $d_{0}$ distribution. The $D^{0}$ impact parameter distribution for one time bin $(5<t / \tau<6)$ is shown in Figure 3.17.

The shapes of the narrow and wide distributions are determined from right-sign data, and the same shapes are used for wrong-sign data. A double Gaussian shape was used for the narrow peak and a single Gaussian shape was used for the wide distribution. The width 


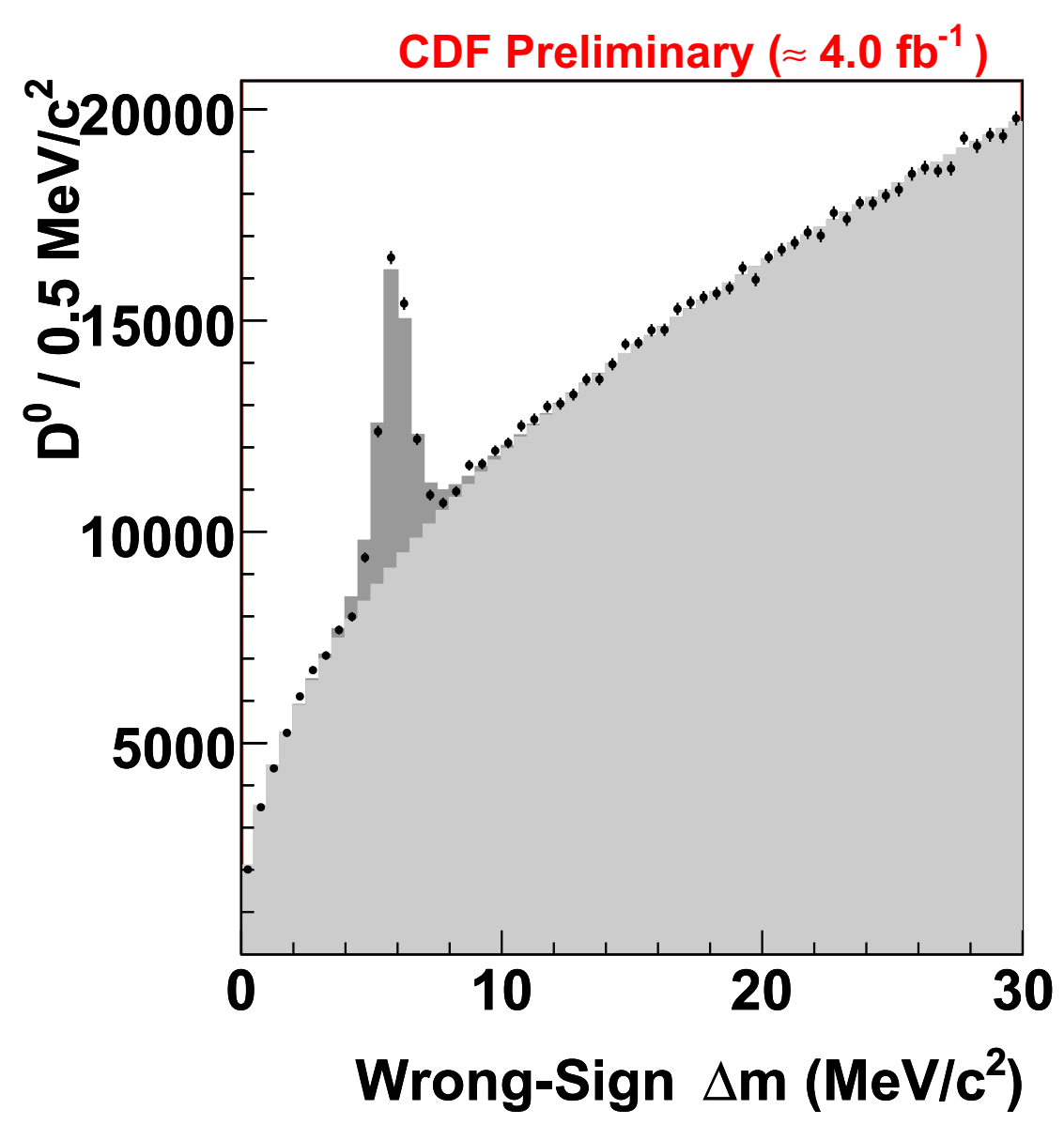

Figure 3.16. Time integrated wrong sign $\Delta M$ distribution.

of the wide distribution increases with time, whereas the width of the narrow distribution is time-independent. The width of the single Gaussian is parametrized from RS data using a polynomial of order 3 . This parametrization is used to obtain the width of the single Gaussian in WS IP distributions.

To determine the prompt RS signal in each time bin, we adopted the following approach:

1. The impact parameter (IP) distribution for each time bin was divided into two regions: $\left|d_{0}\right|<60 \mu \mathrm{m}$ (inner IP region) and $\left|d_{0}\right|>60 \mu \mathrm{m}$ (outer IP region). This cut was chosen to maximize the WS $D^{*}$ yield significance.

2. For a given time bin $i$, we use the single Gaussian shape for the non-prompt distribu- 


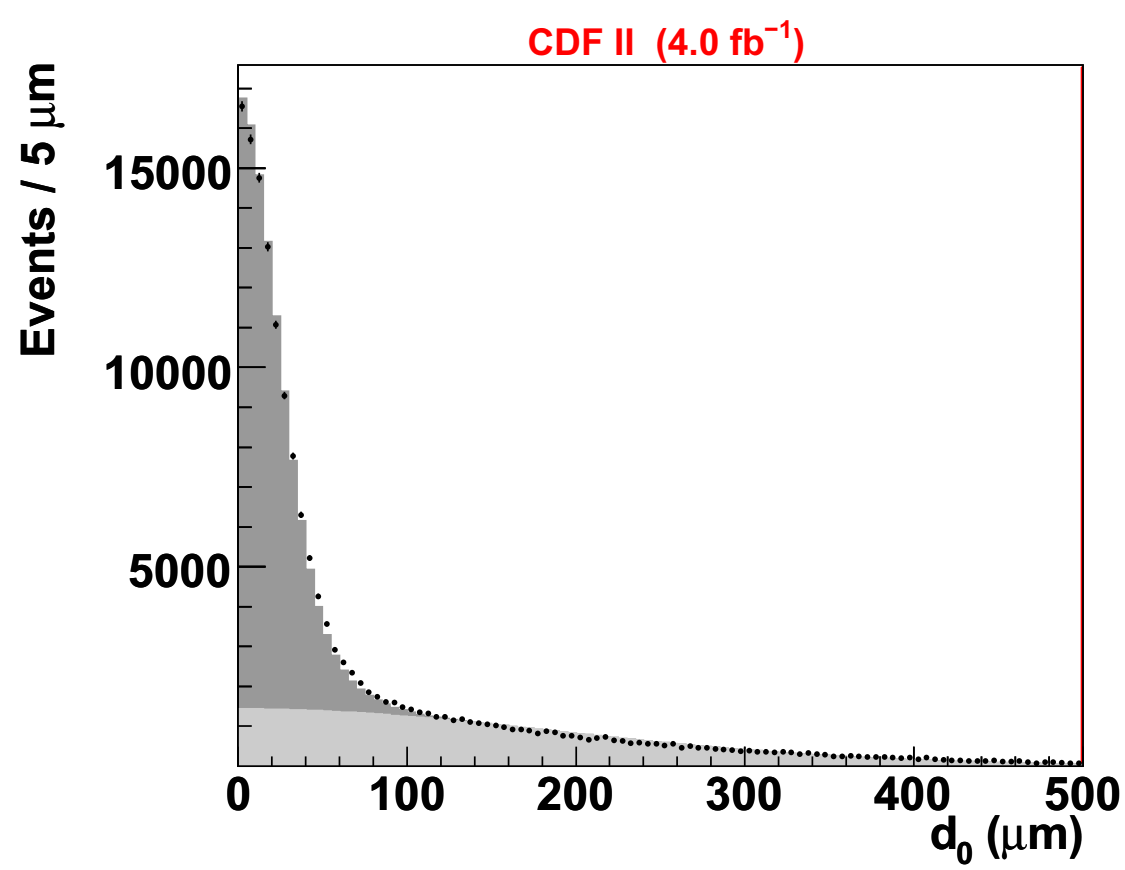

Figure 3.17. Impact parameter distribution for RS $D^{0}$ mesons with $5<t / \tau<6$ using 4.0 $\mathrm{fb}^{-1}$ data.

tion to compute the fraction of events $f_{i}$ as an integral over $0 \leq\left|d_{0}\right| \leq 60 \mu \mathrm{m}$ divided by the integral over the total range $0 \leq\left|d_{0}\right| \leq 500 \mu \mathrm{m}$. Similarly, the fraction of events $g_{i}$ is computed as an integral over $60<\left|d_{0}\right| \leq 500 \mu \mathrm{m}$ divided by the integral over the total range $0 \leq\left|d_{0}\right| \leq 500 \mu \mathrm{m}$.

3. From the prompt distribution shape, we get fractions $f_{p}$ and $g_{p}$ for the two IP regions.

4. The $D^{*}$ yield technique was used to get the number of $D^{*}$ s for inner $\left(n_{i}\right)$ and outer $\left(o_{i}\right)$ IP regions.

5. The number of prompt $\left(N_{P}\right)$ and non-prompt $\left(N_{N P}\right) D^{*}$ s were then computed from the above values as follows:

$$
\begin{gathered}
N_{P}=\frac{n_{i} g_{i}-o_{i} f_{i}}{f_{p} g_{i}-f_{i} g_{p}}, \\
N_{N P}=\frac{o_{i} f_{p}-n_{i} g_{p}}{f_{p} g_{i}-f_{i} g_{p}} .
\end{gathered}
$$


6. The uncertainties on the number of $D^{*}$ s and on the IP fractions were propagated to get the uncertainties on the number of prompt events.

Thus, we obtain the number of prompt RS $D^{*}$ as a function of $D^{0}$ decay time. Using steps 5 and 6 from the above procedure for WS distributions, we obtain prompt WS $D^{*}$ s. The ratio of non-prompt to prompt signal is $\approx 0.02$ at $t / \tau=2$ and this ratio increases with time. At $t / \tau=7$, the ratio is $\approx 1.0$, as was found in the $1.5 \mathrm{fb}^{-1}$ study. The amount of the prompt and non-prompt distributions in each IP region using $4.0 \mathrm{fb}^{-1}$ data is summarized in Table 3.1.

\begin{tabular}{|c|c|c|c|}
\hline \hline Time Bin & Fraction In $\left(f_{i}\right)$ & $\sigma_{f_{i}}$ & Fraction Out $\left(g_{i}\right)$ \\
\hline \hline prompt & & & \\
distribution & 0.95702 & 0.00024 & 0.04298 \\
\hline $0.75-1.00$ & 0.70800 & 0.00271 & 0.29200 \\
$1.00-1.25$ & 0.64350 & 0.00304 & 0.35650 \\
$1.25-1.50$ & 0.58812 & 0.00321 & 0.41188 \\
$1.50-1.75$ & 0.54072 & 0.00329 & 0.45928 \\
$1.75-2.00$ & 0.50007 & 0.00330 & 0.49993 \\
$2.00-2.25$ & 0.46502 & 0.00328 & 0.53498 \\
$2.25-2.50$ & 0.43462 & 0.00323 & 0.56538 \\
$2.50-2.75$ & 0.40809 & 0.00318 & 0.59191 \\
$2.75-3.00$ & 0.38479 & 0.00311 & 0.61521 \\
$3.00-3.25$ & 0.36421 & 0.00304 & 0.63579 \\
$3.25-3.50$ & 0.34593 & 0.00298 & 0.65407 \\
$3.50-3.75$ & 0.32963 & 0.00292 & 0.67037 \\
$3.75-4.00$ & 0.31501 & 0.00285 & 0.68499 \\
$4.0-4.5$ & 0.29576 & 0.00277 & 0.70424 \\
$4.5-5.0$ & 0.27418 & 0.00267 & 0.72582 \\
$5.0-5.5$ & 0.25631 & 0.00259 & 0.74369 \\
$5.5-6.0$ & 0.24137 & 0.00252 & 0.75863 \\
$6.0-7.0$ & 0.22327 & 0.00244 & 0.77673 \\
$7.0-8.0$ & 0.20521 & 0.00238 & 0.79479 \\
$8.0-10.0$ & 0.18744 & 0.00239 & 0.81256 \\
\hline \hline
\end{tabular}

Table 3.1. Prompt and non-prompt distribution fractions using $4.0 \mathrm{fb}^{-1}$ data. $\sigma_{f_{i}}$ is the uncertainty on $f_{i}$ based on the uncertainties of the parametrization for the non-prompt Gaussian width.

This completes the illustration of the $D^{*}$ yield technique. The total number of $K \pi$ mass fits equals $60(\Delta M$ bins $) \times 20($ time bins $) \times 2($ IP regions $) \times 2($ RS and WS events $)=48000$ fits. 


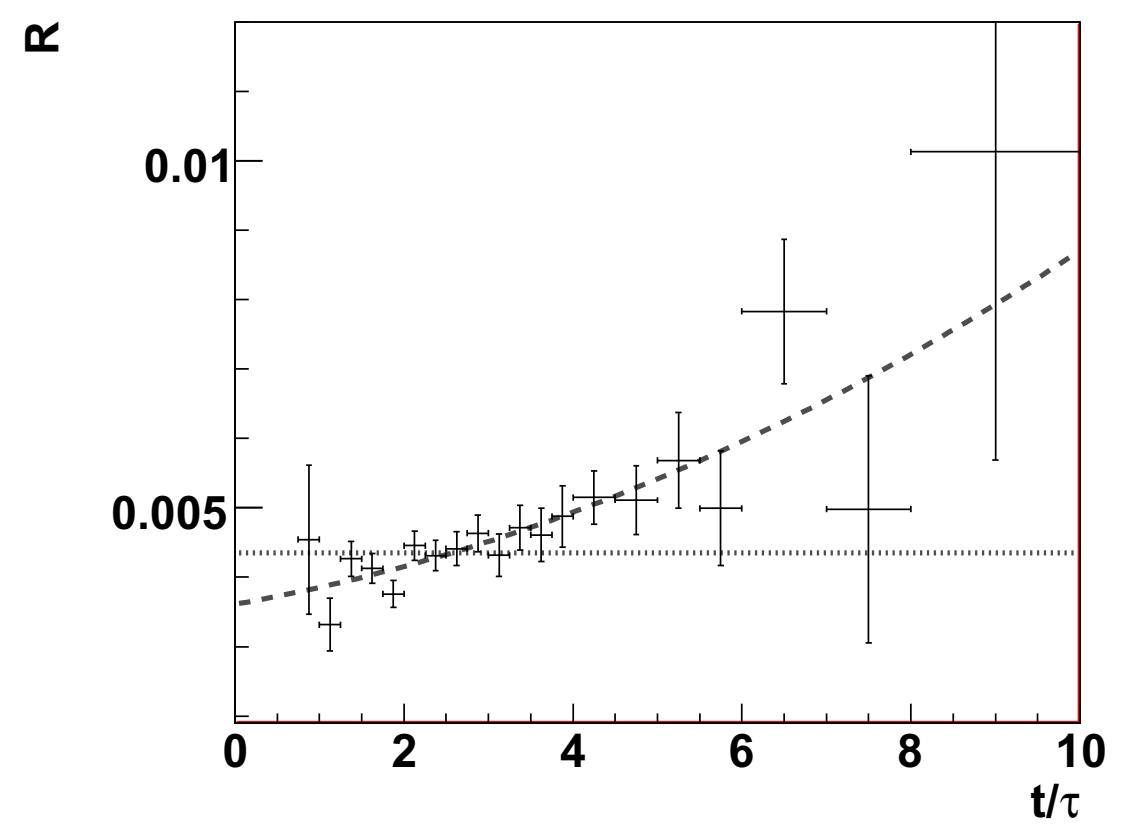

Figure 3.18. The ratio of wrong sign to right sign $D^{*}$ s as a function of normalized $D^{0}$ decay time $\left(4.0 \mathrm{fb}^{-1}\right)$.

\subsection{Results}

\subsubsection{WS/RS Ratio}

The ratio of wrong sign to right sign prompt $D^{*}$ in 20 time bins is summarized in Table 3.2 and plotted in Figure 3.18. The large uncertainties at small $t / \tau$ are due to the trigger turn on. The large uncertainties appearing at larger $t / \tau$ are due to the exponential decays. We fit the time dependent WS/RS ratio using the fit function given by Equation 3.1. The dashed curve is from a least-square fit with $x^{2}$ and $y^{\prime}$ allowed to vary. This fit determines the mixing parameters. The $\chi^{2}$ for this fit is 19.2. The dotted line is the fit assuming no-mixing $\left(x^{\prime 2}=y^{\prime}=0\right)$. The $\chi^{2}$ for this fit is 36.8 .

The difference between the $\chi^{2}$ s of the best fit and the no-mixing fit clearly favors the mixing fit. This difference can be used to cross check the final result of mixing significance. The results of the two fits are given in Table 3.3. The variables $x^{\prime 2}$ and $y^{\prime}$ are highly correlated with covariance $\rho=-0.976$. 


\begin{tabular}{|c|c|}
\hline Time Bin & WS/RS Ratio \\
\hline $0.75-1.00$ & $4.54 \pm 1.074$ \\
$1.00-1.25$ & $3.32 \pm 0.38$ \\
$1.25-1.50$ & $4.26 \pm 0.25$ \\
$1.50-1.75$ & $4.13 \pm 0.21$ \\
$1.75-2.00$ & $3.76 \pm 0.19$ \\
$2.00-2.25$ & $4.45 \pm 0.21$ \\
$2.25-2.50$ & $4.31 \pm 0.22$ \\
$2.50-2.75$ & $4.41 \pm 0.24$ \\
$2.75-3.00$ & $4.63 \pm 0.26$ \\
$3.00-3.25$ & $4.32 \pm 0.31$ \\
$3.25-3.50$ & $4.71 \pm 0.32$ \\
$3.50-3.75$ & $4.61 \pm 0.39$ \\
$3.75-4.00$ & $4.88 \pm 0.44$ \\
$4.00-4.50$ & $5.15 \pm 0.39$ \\
$4.50-5.00$ & $5.11 \pm 0.49$ \\
$5.00-5.50$ & $5.68 \pm 0.69$ \\
$5.50-6.00$ & $4.99 \pm 0.83$ \\
$6.00-7.00$ & $7.83 \pm 1.04$ \\
$7.00-8.00$ & $4.98 \pm 1.92$ \\
$8.00-10.00$ & $10.13 \pm 4.45$ \\
\hline
\end{tabular}

Table 3.2. WS/RS ratios for $4.0 \mathrm{fb}^{-1}$ data.

\begin{tabular}{|c|c|c|c|c|}
\hline Fit & $R_{D}\left(10^{-3}\right)$ & $y^{\prime}\left(10^{-3}\right)$ & $x^{2}\left(10^{-3}\right)$ & $\chi^{2} / \mathrm{ndf}$ \\
\hline Best fit(unconstrained) & $3.61 \pm 0.42$ & $3.5 \pm 4.8$ & $0.11 \pm 0.21$ & $15.3 / 17$ \\
No-mxing $\left(x^{\prime 2}=y^{\prime}=0\right)$ & $4.35 \pm 0.07$ & 0.0 & 0.0 & $46.7 / 19$ \\
\hline
\end{tabular}

Table 3.3. The results of the WS/RS ratio fits $\left(4.0 \mathrm{fb}^{-1}\right.$ data).

\subsubsection{Systematic Uncertainties}

Systematic uncertainties on the fit shapes arise because the true distribution shapes could be complicated, but well matched by simple functions that we have chosen. Since the fit parameters in the fit shape are floating (allowed to vary), the statistical uncertainties include shape uncertainties. This is not true if the parameters are fixed beforehand from other sources, for example from Monte Carlo or from time integrated data.

In the ratio plot, the uncertainties for each bin include contributions from statistical and systematic uncertainties. Most systematic uncertainties, such as the detector time resolution, cancel in WS/RS ratio.

The significant systematic uncertainties arise from the background shapes in $K \pi$ mass distributions and in mass difference distributions. 
The following sections examine the possible sources of systematic uncertainties. These studies were made using $\mathrm{RS} D^{*} s$ from the signal region in the $\Delta M$ distribution, with $4.0<\Delta M<9.0 \mathrm{MeV} / c^{2}$ and the impact parameter region $\left|d_{0}\right|<60 \mu \mathrm{m}$.

\section{Signal Shapes}

The time dependent signal shapes used to fit the $K \pi$ mass distributions and the $\Delta M$ distributions were fixed from the time integrated RS distributions. Since RS and WS decays have the same kinematics, they have the same distribution. Any systematic uncertainties in RS and WS distributions will cancel in the ratio. So, there is no additional systematic uncertainty assigned due to signal shapes.

\section{Background shape in $K \pi$}

Since we allow the quadratic polynomial parameters to float for every $K \pi$ fit, the systematic uncertainties are already included in the statistical uncertainties returned by the fitter. To cross check the possibility of having an alternate fit function available for the background shape, we tried adding an extra third order term in the polynomial. This did not result in a significant change in the signal yield and we observed that the third order term was set to zero in all the fits.

In Figure 3.19 showing the RS $K \pi$ distribution, the background fit suggests that an additional Gaussian "lump" is needed at $1.83 \mathrm{GeV} / c^{2}$. A fit for the right sign $K \pi$ plot has a $\chi^{2} /$ ndf of 24.2 with quadratic background without an additional Gaussian. With addition of the Gaussian, the $\chi^{2} /$ ndf changes to 1.89 . Figure 3.19 shows the quadratic fit with and without the extra Gaussian. This additional Gaussian is required only in RS and is very small in amplitude compared to the signal amplitude. In the WS distribution, the amplitude of the extra Gaussian was essentially zero.

As will be discussed in Appendix C.2, we did not find a source for the lump at 1.83 $\mathrm{GeV} / c^{2}$. We therefore assign a small systematic uncertainty to the final result. We treat the lump as a part of the background shape. If we treat the lump as a part of signal shape, it changes the WS/RS ratio by $0.07 \%$. A systematic uncertainty of the same amount was added in each time bin. 


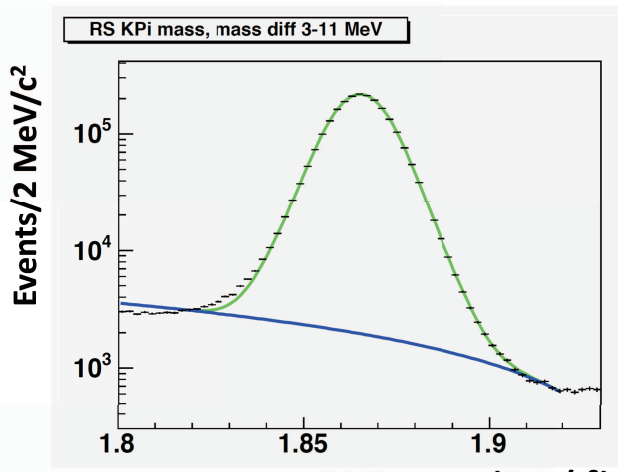

(a)
$\mathrm{RS} K \pi$ mass $\left(\mathrm{GeV} / \mathrm{c}^{2}\right)$

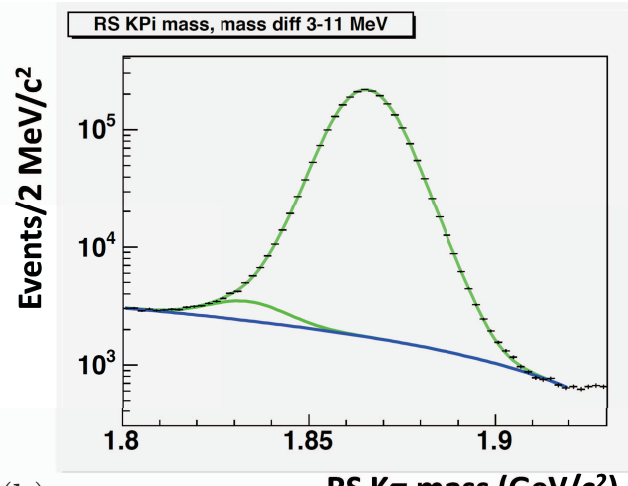

(b)

RS $K \pi$ mass $\left(\mathrm{GeV} / \mathrm{c}^{2}\right)$

Figure 3.19. RS $K \pi$ mass distribution with quadratic fit function for background (a) without additional Gaussian and (b) with extra Gaussian at $1.83 \mathrm{GeV} / c^{2}$. The signal shape is a double Gaussian. These plots were made using $1.5 \mathrm{fb}^{-1}$ data [44].

\section{Non-prompt $D^{*}$ distribution}

The width of the non-prompt single Gaussian in the IP distribution is obtained from RS data. To check for the systematic effects arising from fixing the width from RS data, we vary the width by $\pm 1 \sigma$ and observe the change in the WS/RS ratio. The effect was observed to be negligible.

\section{Background in Mass Difference Distribution}

In the mass difference distribution, we have a power term of $\Delta M$ in the background shape. This term was fixed from the time integrated WS distribution. The change in the power term by $\pm 1 \sigma$ resulted in a change to the WS/RS ratio in each time bin. On average, we observe an effect of $0.46 \%$. We add a systematic uncertainty of this amount in each time bin.

\subsubsection{Mixing Significance}

The mixing significance is the consistency of the data with the null hypothesis i.e. nomixing or $x^{\prime 2}=y^{\prime}=0$. We interpret the mixing significance using Bayesian probability contours in $x^{\prime 2}-y^{\prime}$ space, with $R_{D}$ treated as a nuisance parameter. The nuisance parameter is the variable whose values are known with some limited accuracy but they are not of immediate interest. However, they must be accounted for the measurement of the variables 
of interest [46].

For a data represented by vector $x$ and for a set of parameters defined by $\phi$, Bayes theorem gives the posterior probability $p(\phi \mid x)$ by

$$
p(\phi \mid x)=\frac{\mathcal{L}(x \mid \phi) \pi(\phi)}{\int \mathcal{L}\left(x \mid \phi^{\prime}\right) \pi\left(\phi^{\prime}\right) d \phi^{\prime}}
$$

where $\pi(\phi)$ is the prior probability distribution, the denominator $m(\phi)=\int \mathcal{L}\left(x \mid \phi^{\prime}\right) \pi\left(\phi^{\prime}\right) d \phi^{\prime}$ is called the marginal distribution that ensures that the probability is normalized, and $\mathcal{L}(x \mid \phi)$ is the likelihood function related to the $\chi^{2}$ by the relation

$$
\mathcal{L}(x \mid \phi)=e^{-\chi^{2}(x \mid \phi) / 2}
$$

Given the posterior probability $p(\phi \mid x)$, one can choose a region $D$ for which

$$
\int_{D} p(\phi \mid x) d \phi=\alpha
$$

where $\alpha$ is the desired significance level.

From the WS/RS ratio plot, for a time bin $i$, we have a measured ratio $R_{i}$ with uncertainty $\sigma_{i}$. With $\phi$ being the set of given mixing parameters that we wish to test, and $E(\phi, i)$ the predicted ratio from Equation 3.1, the $\chi^{2}$ is given by

$$
\chi^{2}=\Sigma \frac{\left[R_{i}-E(\phi, i)\right]^{2}}{\sigma_{i}^{2}}
$$

Using this $\chi^{2}$, we can calculate the likelihood $\mathcal{L}\left(R_{i} \mid \phi\right)$ and then the posterior probability $p\left(\phi \mid R_{i}\right)$ from Bayes theorem. We assume a flat prior probability of $x^{\prime 2}$ and $y^{\prime}$ variables.

Since our likelihood distribution is a grid of possible $\phi$ values in $x^{\prime 2}-y^{\prime}$ space, we accumulate a set of points in region $D$ until the sum of all the probabilities equals $\alpha$. We add points such that the probability $p\left(\phi \mid R_{i}\right)$ for every point within the interval $D$ is higher than every point outside the interval.

Figure 3.20 shows the 1 to $6 \sigma$ Bayesian probability contours. The no-mixing point $\left(x^{\prime 2}=y^{\prime}=0\right)$ falls on the 5.3 sigma contour. In other words, no-mixing is excluded with 


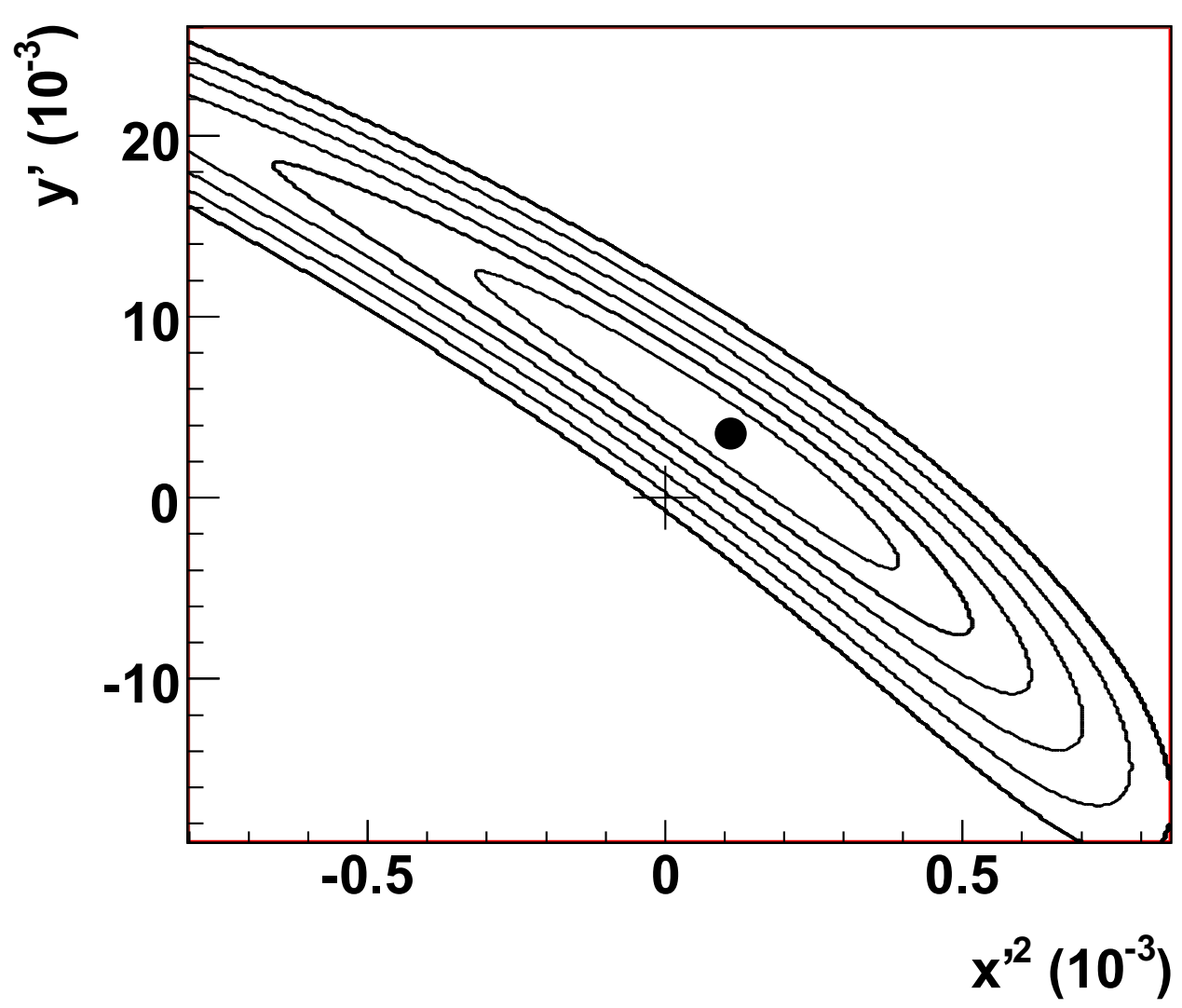

Figure 3.20. Bayesian probability contours equivalent to 1 to 6 Gaussian standard deviation. The no-mixing point $\left(x^{\prime 2}=y^{\prime}=0\right)$, shown as a cross, falls on the $5.3 \sigma$ contour. In other words, our result excludes no-mixing hypothesis with 5.3 Gaussian standard deviation. The solid dot shows the measured values of $x^{\prime 2}$ and $y^{\prime}$ from the best fit.

5.3 Gaussian standard deviations. This corresponds to an observation of $D^{0}-\bar{D}^{0}$ mixing in $D^{0} \rightarrow K \pi$ channel using the CDF II detector.

\subsection{Cross-check of Mixing Significance}

We use three methods to cross check the observed mixing significance:

- The difference in log likelihood $\left(\Delta \chi^{2}\right)$ between the mixing and no-mixing fits.

- The $\Delta \chi^{2}$ distribution from an ensemble of simulated WS/RS ratios.

- Bayesian contours from a simulated WS/RS ratio. 
The difference between the $\chi^{2}$ s of the mixing fit and no-mixing fit gives a significance of 5.2 Gaussian standard deviations for 2 degrees of freedom. This is consistent with the observed significance from the Bayesian contours. The calculations are based on the incomplete Gamma function $\mathrm{P}(a, x)$ where $a=\operatorname{ndf} / 2, x=\chi^{2} / 2$, and the variable $\mathrm{P}(a, x)$ is the probability that the observed $\chi^{2}$ for a correct model should be less than the value of $\chi^{2}$.

To simulate the WS/RS ratios, we use the central values of the mixing parameters from the $1.5 \mathrm{fb}^{-1}$ data. On each data point in the $1.5 \mathrm{fb}^{-1}$ ratio plot, we add random noise from a Gaussian distribution having standard deviation equal to the uncertainty on the data point in the corresponding time bin in the $4.0 \mathrm{fb}^{-1}$ ratio plot. We fit the simulated ratio plot with the mixing Equation 3.1 in two ways: with mixing and without mixing, and compute the $\Delta \chi^{2}$ for these fits.

We make a thousand such simulations to get a $\Delta \chi^{2}$ distribution. Using the numerical mean of the $\Delta \chi^{2}$ distribution we compute the significance as we did for the observed $\Delta \chi^{2}$.

The top plot in Figure 3.21 shows one simulation in which the dashed curve is the best fit and the solid line corresponds to no mixing with $x^{\prime 2}$ and $y^{\prime}$ fixed to zero. The bottom plot in Figure 3.21 shows a $\delta \chi^{2}$ distribution from one thousand simulated experiments. The numerical mean of this distribution is 30.36 which corresponds to a significance of 5.15 Gaussian standard deviations for 2 degrees of freedom. This value is consistent with our observed value.

In the third cross-check, we compute Bayesian probability contours for a single simulated experiment. The Bayesian contours are computed in the same way as was done for the real data. Figure 3.22 shows the result of one such experiment. In the resulting plot, the no-mixing point lies on the 5.0 sigma contour. 

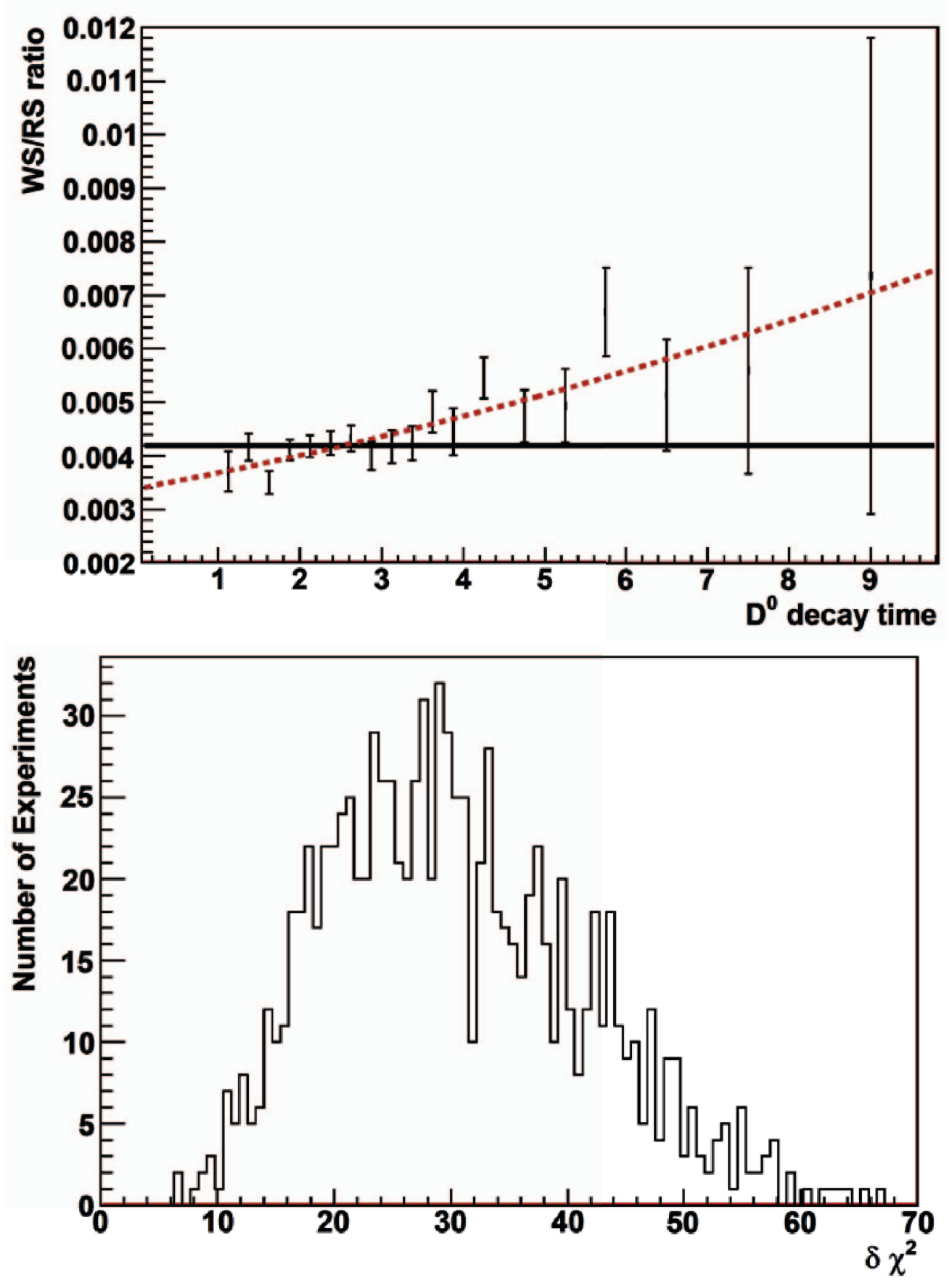

Figure 3.21. The simulated experiment and $\delta \chi^{2}$ distribution: the top plot shows a single simulation of a WS/RS ratio. The bottom plot shows the $\delta \chi^{2}$ distribution from 1000 simulated experiments. 


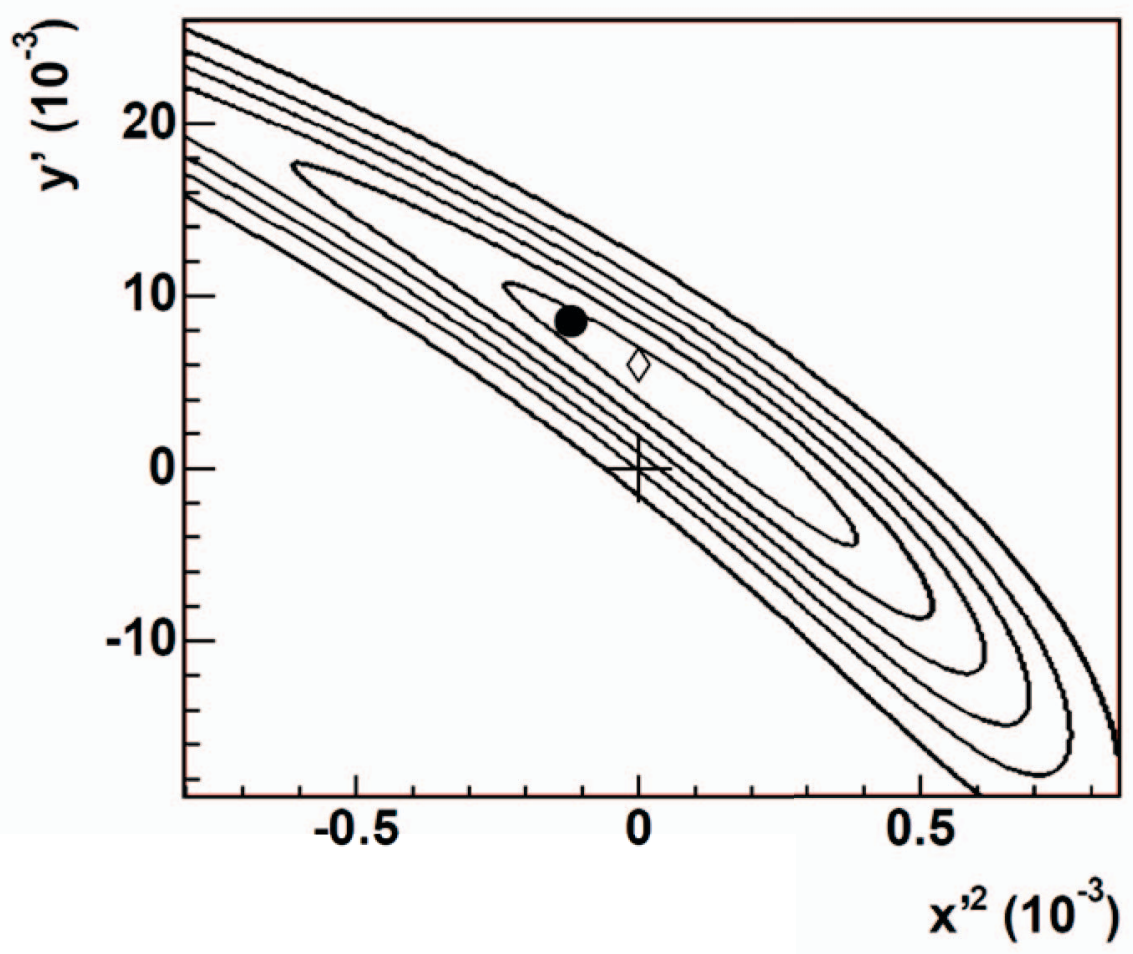

Figure 3.22. Bayesian contours from a simulated experiment. The no-mixing point is on the $5.0 \sigma$ contour. The solid dot shows the measured values of $x^{\prime 2}$ and $y^{\prime}$ from the best fit. The diamond shows the highest probability point in physically allowed $x^{\prime 2}$. 


\section{CHAPTER 4}

\section{SUMMARY AND FUTURE PROSPECTS}

We presented the first observation of $D^{0}-\bar{D}^{0}$ mixing in a single analysis. We measured the ratio of doubly Cabibbo suppressed or WS $\left(D^{0} \rightarrow K^{+} \pi^{-}\right)$decay rate to Cabibbo favored or RS $\left(D^{0} \rightarrow K^{-} \pi^{+}\right)$decay rate as a function of $D^{0}$ decay time. We used an integrated luminosity of $4.0 \mathrm{fb}^{-1}$ of proton anti-proton collisions at $\sqrt{s}=1.96 \mathrm{TeV}$ at the collider detector at Fermilab (CDF II).

A signal of $24000 D^{0} \rightarrow K^{+} \pi^{-}$decays was obtained using the $D^{*}$ yield technique that was presented in Section 3.6.3. We measured the $D^{0}-\bar{D}^{0}$ mixing parameters $R_{D}, y^{\prime}$, and $x^{\prime 2}$ from the WS/RS ratio. Using the Bayesian probability contours, we found that the no-mixing hypothesis was excluded with a probability equivalent to 5.3 Gaussian standard deviations. The probabilities from the frequentist cross-checks were consistent with the probabilities from the Bayesian contours.

Table 4.1 summarizes the results from the BaBar, Belle, and CDF II experiments. Figure 4.1 shows the approximate standard error ellipses for the three experiments to help us visually compare the three results. These error ellipses were obtained as described in Section 1.4 using the fit error matrices of the BaBar, Belle, and current CDF results.

This analysis was performed assuming no $\mathrm{CP}$ violation. This allowed us to combine $D^{*+}$ and $D^{*-}$ decays. The CP violation study in $D^{0}-\bar{D}^{0}$ mixing can be performed in the future. For CP violation study, the same analysis can be performed to measure the mixing parameters separately for $D^{*+}$ and $D^{*-}$ data. However, since kaon and pion have different absorption cross-sections in the detector material, we need to determine the corrections on the mixing parameters. 


\begin{tabular}{|c|c|c|c|}
\hline \hline Experiment & $R_{D}\left(\times 10^{-3}\right)$ & $y^{\prime}\left(\times 10^{-3}\right)$ & $x^{\prime 2}\left(\times 10^{-3}\right)$ \\
\hline BaBar & $3.03 \pm 0.16 \pm 0.10$ & $9.7 \pm 4.4 \pm 3.1$ & $-0.22 \pm 0.30 \pm 0.21$ \\
Belle & $3.64 \pm 0.17$ & $0.6 \pm 4.0 \pm 3.9$ & $0.18 \pm 0.21 \pm 0.23$ \\
CDF & $3.04 \pm 0.55$ & $3.5 \pm 4.8$ & $0.11 \pm 0.21$ \\
\hline \hline
\end{tabular}

Table 4.1. The $D^{0}-\bar{D}^{0}$ mixing parameters provided by BaBar and Belle experiments compared with the new CDF result.

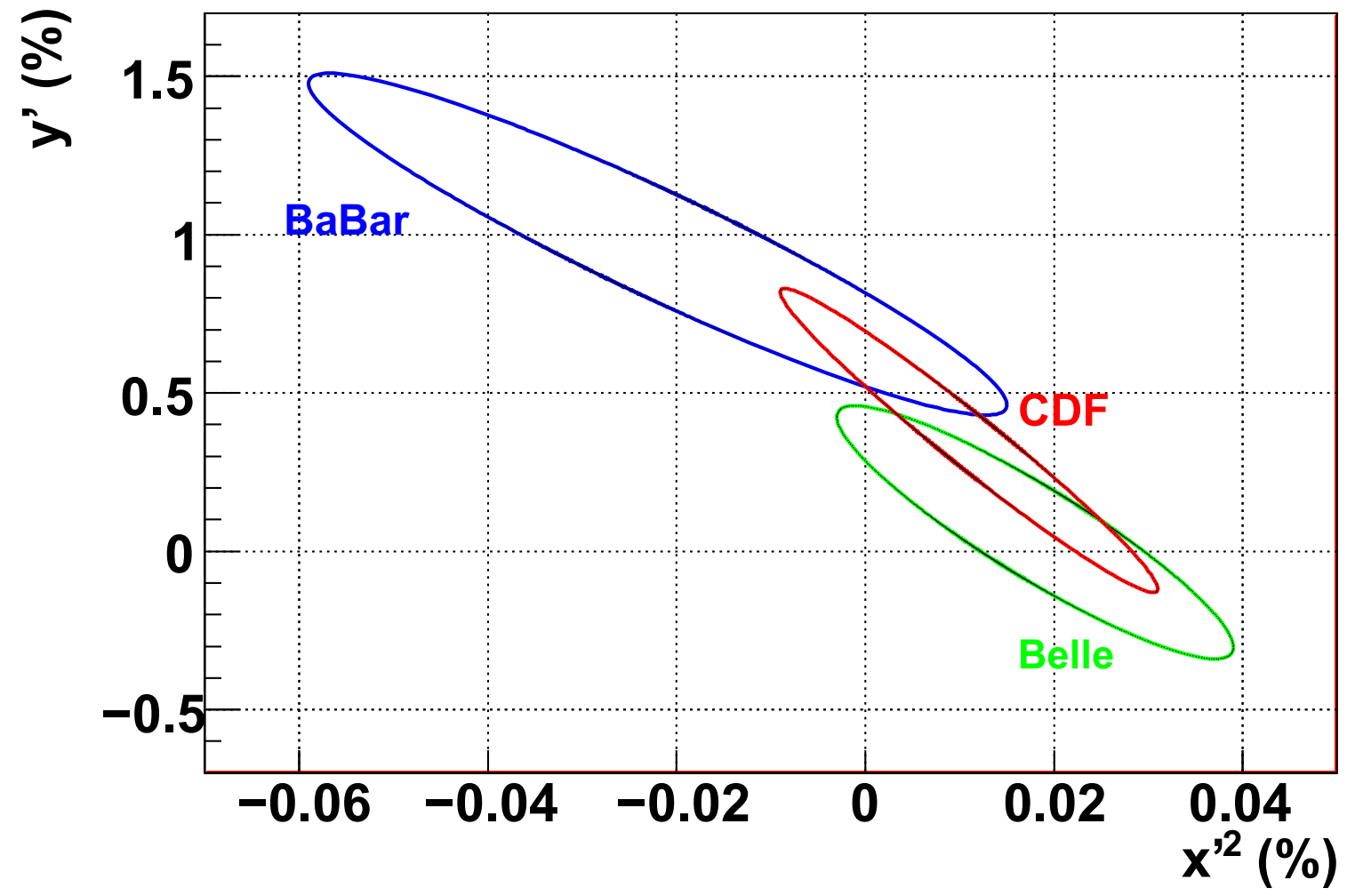

Figure 4.1. Visual comparison of the Belle, BaBar, and the new CDF II results. The plot shows standard error ellipses obtained from the fit error matrices. The BaBar and Belle measurements are the same as in Figure 1.6. The new CDF II measurement is centered around $x^{\prime 2}=0.011 \%$ and $y^{\prime}=0.35 \%$. 


\section{Appendix A}

\section{ARTIFICIAL NEURAL NETWORK METHOD}

In our efforts to improve the results of the $D^{0}-\bar{D}^{0}$ mixing analysis, we attempted the application of Artificial Neural Network method. This method is described in this section.

\section{A.1 Neural network method for $D^{0}-\bar{D}^{0}$ mixing analysis}

We discussed four types of backgrounds and the related cuts to clean up those backgrounds in Section 3.6.1. Even after applying all the cuts, the data can still have some random pion and combinatorial backgrounds. We attempted to use Artificial Neural Network methods to reduce these backgrounds.

The belief that a neural network can reduce combinatorial and random pion backgrounds was based on the neural network's ability to learn a complicated, non-linear, multidimensional discrimination function in the hyper surface of its input parameter space. Neural networks make the signal and background discrimination possible by reducing multidimensional cuts to a one dimensional neural-network output cut. This can bring an improvement in the signal significance over the standard cut optimization method.

The artificial neural network algorithm finds a non-linear discrimination function by adjusting hundreds of internal parameters. The algorithm can be graphically visualized as a set of input nodes, middle layers (called hidden layers), connecting links called synapses and output nodes, as shown in Figure A.1. The algorithm starts with a random weight assigned to each synapse. At each subsequent layer, the linear combination of the sigmoid functions is computed. The sigmoid function is defined as

$$
\sigma(x)=\frac{1}{1+e^{x}}
$$

where $x$ is the input on a synapse. The sigmoid function has an "S" shape lying between 0

and +1 . The function can be modified to have a range of -1 to +1 . The linear combination 


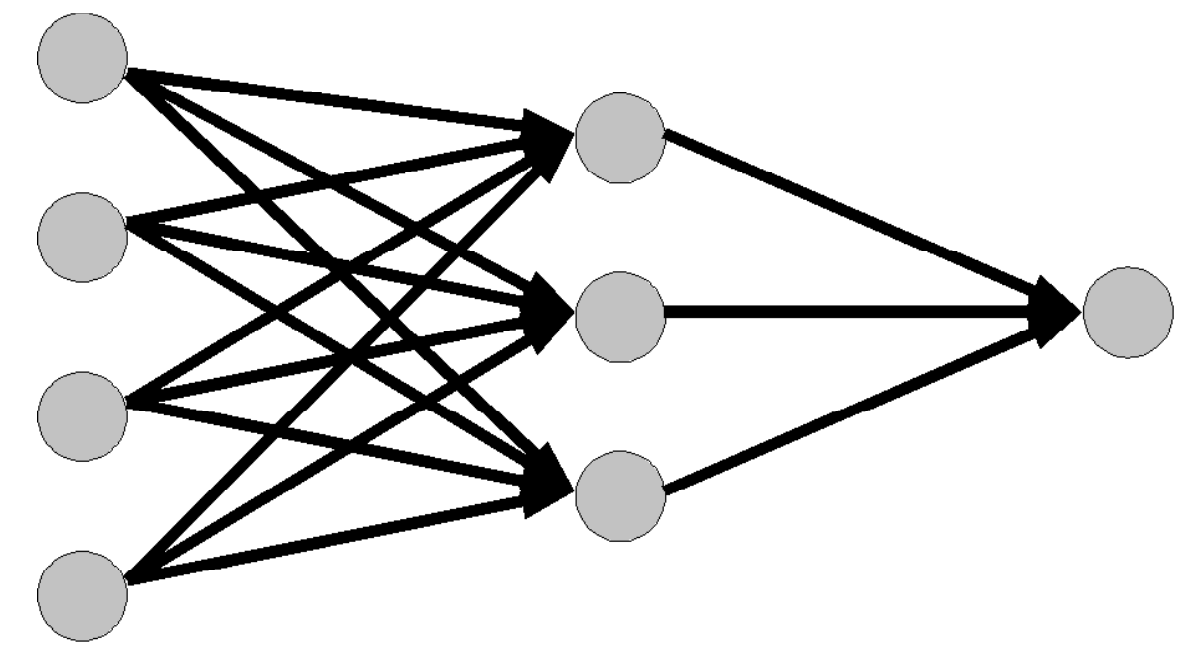

Figure A.1. A typical artificial neural network has input nodes, synapses, middle layer with hidden nodes, and output node. In the above sketch, the nodes are shown by circles while the arrows are synapses. The network finds a discrimination function by adjusting weights on each of the synapses.

of these sigmoid functions will be a non-linear function. If, as is usual in particle physics, the problem is a classification problem, then only one output node is required. The output at this node will be between -1 and +1 . If the neural network is used to find a probability distribution function, then more than one output node may be required. There are no specific rules to decide the number of hidden layers and the number of nodes in each hidden layer. In most cases, only one hidden layer is sufficient and the rule of thumb says that the number of nodes in the hidden layer should be equal to twice the number of input variables.

In the training stage, a set of input values from a known data sample is given to the input nodes of the neural network. The target output is set to +1 for signal and -1 for background. If the neural network output at the end node is different from the target output, then the difference is back-propagated and the weights at all the synapses are adjusted to reduce the error. Several error minimization algorithms are available and the choice of algorithm depends on the type of problem. The learning process is repeated for all sets of inputs until the weights on the synapses become stable. At this stage, the network is said to be "trained" and can be used for signal and background separation in new data.

For our purpose, we used a commercial neural network software product called NeruoBayes [47] which is made available for CDF users under a special license. 


\section{A.2 Training sample preparation}

For training the neural network, we needed signal and background samples. Many neural network analyses use Monte Carlo generated training samples. But, since in our case, it is difficult to simulate all significant backgrounds, the background samples are taken from real data.

We used RS signal as our training sample. The RS signal was extracted from the data requiring $\left|\mathrm{m}_{D^{0}}\right|<20 \mathrm{MeV} / c^{2}$ and $\Delta M<20 \mathrm{MeV} / c^{2}$. We also applied standard $\mathrm{dE} / \mathrm{dx}$ and impact parameter cuts on the data.

For the background training sample, we extracted random pion and combinatorial backgrounds from the WS sample. To extract the background sample, we applied the $\mathrm{dE} / \mathrm{dx}$ cut described in Section 3.6.1, but not the impact parameter cut. We required the wrong sign $K \pi$ mass to lie between either 1.8 and $1.835 \mathrm{GeV} / c^{2}$ or 1.895 and $1.928 \mathrm{GeV} / c^{2}$. A wrong sign mass difference $\Delta M_{D^{*}}(\mathrm{WS})>30 \mathrm{MeV} / c^{2}$ was required to remove most signal events, keeping the random pion background. In addition, we applied a right sign mass cut of $\left|\mathrm{m}_{K \pi}-\mathrm{m}_{D^{0}}\right|<70 \mathrm{MeV} / \mathrm{c}^{2}$ to eliminate the mis-identification background. The final regions in the mass distributions from where the signal and background are extracted can be best illustrated by Figure A.2. The central green area indicates the signal region and the four red boxes indicate the background region. Even with a very narrow signal region, we get 8 to 10 times larger signal than background.

\section{A.3 Input parameters and settings}

We followed a rule that the variables, such as $D^{0}$ mass, $D^{*}$ mass difference, $\mathrm{dE} / \mathrm{dx}$ and impact parameter of $D^{0}$, that used to obtain the training data would not be used as inputs to the neural network. The reason is that there is nothing for the neural network to learn from these already used variables. For the rest of the available variables in our mini-data set, we studied the distribution for signal and background for each variable and decided to keep the variable as a neural network (NN) input only if there is a significant difference between the two distributions.

Following this process, we finalized twelve variables as follows: 


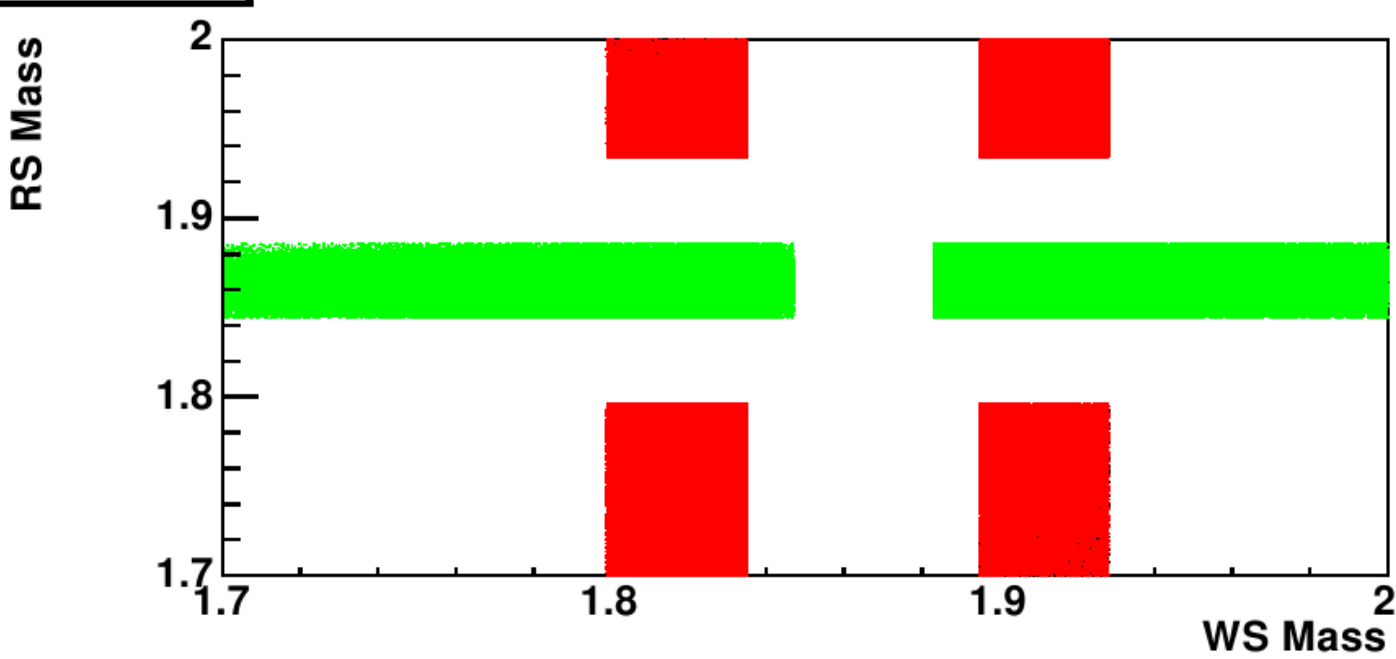

Figure A.2. The RS and WS $K \pi$ mass in $\mathrm{GeV} / c^{2}$ plotted together to illustrate signal and background samples used for neural network training. The red boxes show the region from where background sample is extracted. The central green area shows the signal region.

We tried impact parameter of soft pion, transverse momentum and Z0 variables (the distance of the closest approach along the beam line) for kaon, pion, and the tagging pion. Transverse and $z$ components of the decay length significance of the $D^{0}$ were also considered.

We also considered an isolation variable for the $D^{0}$ meson. The definition of isolation is given in Appendix B.1. In addition, we used the $\eta$ variables for the tagging pion and the $D^{0}$. The signal and background distributions for kaon and pion $\eta$ variables were not significantly different, and so they are not used as neural network inputs. Apart from these 12 variables, NeuroBayes requires one extra unnamed input variable for internal use.

The plots for the signal and background distributions for these variables are shown in Figures A.5 through A.15. As can be seen from these plots, some variables such as transverse momentum of kaons and pions have large differences in their signal and background distributions. We expect the neural network to learn more from these inputs than from others. The output of the neural network training provides information about the final weights assigned to each input synapse. This information is consistent with our expectation that the synapses corresponding to kaon and pion transverse momentum inputs were always given relatively higher weights. Interestingly, we found that the $D^{0}$ isolation variable which we found not very useful in the standard analysis was also given significant weight and 
removing it from the neural network inputs reduced the neural network's discrimination power.

Since the purpose of the neural network in our case was to separate signal from background, this becomes a classification problem and so the neural network was designed to have a single output node. The default setting of the NeuroBayes program uses only $80 \%$ of the training sample events for training and leaves $20 \%$ for testing. This setting was kept unchanged. The number of epochs was set to 200, where an epoch is the number of iterations or events after which the weights are updated. This number was obtained by trial and error to maximize the learning speed. The number of training iterations was set to 15. The error minimization method used was BFGS ( Broyden, Fletcher, Goldfarb, and Shanno) learning method. The other default settings were kept unchanged. For 12 input variables, we set 24 nodes in the hidden layer and one node in the output layer.

The teacher program reads events from the signal and background data files that prepared for training. Only 1.5 million total (signal + background) events are allowed for the training. Since we have very few background events compared to signal events, we wanted to use all the available background events. For $2.5 \mathrm{fb}^{-1}$ data, we have a total of 5.3 million signal events and 0.65 million background events. We used all the 0.65 million background events and only 0.85 million signal events in order to maintain the total number of 1.5 million events. The signal to background ratio was then 1.3.

To train the neural network, we provided it with signal events with target output value set to +1.0 and background events with the target output value set to -1.0 . The neural network then started the training process and after it was complete, the configuration (which includes the final weights on synapses) was stored. The executable also produces histograms of output node value (NN output) for each event in the signal and background training sample. Figure A.3 shows the training output in which signal is shown in red and background is shown in black. We observe that the signal gradually increases and the background decreases with NN output from -1.00 to +1.00 . From Figure A.3, we expected to get maximum integrated signal and minimum integrated background if we required the NN output to be slightly greater than 0.0 .

The NN executable applies the stored configuration on the new data which we wanted 


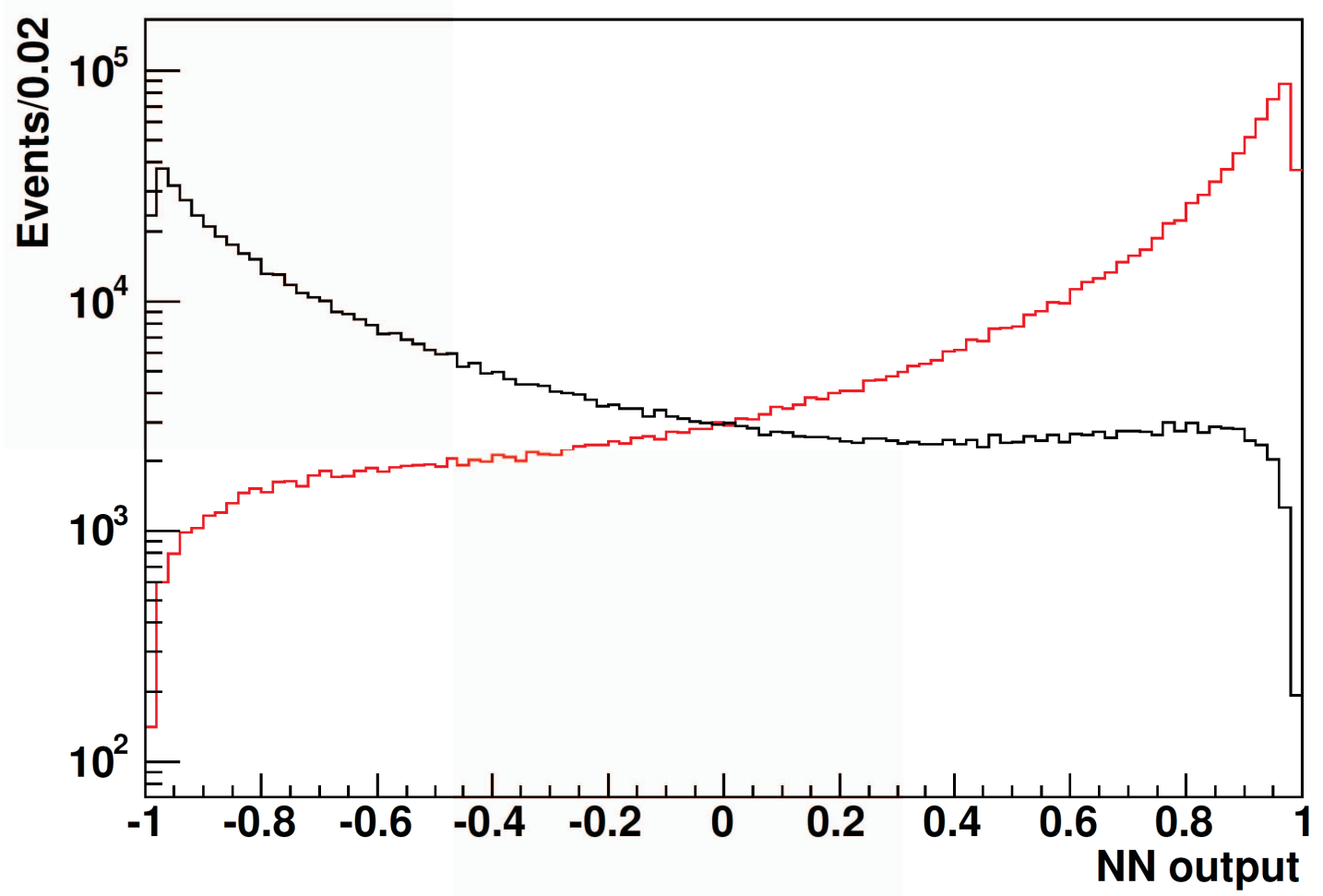

Figure A.3. Neural network training output. Signal is shown in red color and background in black color. Signal gradually increases and background decreases with NN output. Maximum signal significance is found at NN cut $>0.10$.

to classify. The executable takes the input variables from the new data in the same order as they were given for training and computes the NN output value for each event using the saved configuration.

The optimal neural network cut was chosen to maximize the WS $D^{*}$ yield significance. We applied a neural network cut for which we expected to get the maximum signal to background ratio. The data passing that cut was used to find the WS $D^{*}$ yield using the yield technique described previously. The yield technique gives the number of WS $D^{*}$ as a value of the first fit parameter. The uncertainties $\delta$ on the first parameter can be considered as equivalent to $\sqrt{(S+B)}$, where $\mathrm{S}$ is signal and $\mathrm{B}$ is background. We used the ratio of yield/ $\delta$ to compare the NN result with the result from the standard analysis method. Table A.1 shows the WS $D^{*}$ significance we got from the standard method for various neural network cuts. We see from the table that we got the maximum signal significance for the neural network cut of 0.10 . This was close to the expected NN cut value. 
66

\begin{tabular}{|l|c|c|c|}
\hline Analysis & WS $D^{*}$ yield & Uncertainty $\delta$ & Ratio:Yield $/ \delta$ \\
\hline Standard method & 24056.82 & 374.40 & 64.25 \\
NN cut $>0.0$ & 24363.45 & 376.61 & 64.69 \\
NN cut $>0.05$ & 238888.9 & 368.61 & 64.80 \\
NN cut $>0.10$ & 23481.15 & 360.88 & 65.06 \\
NN cut $>0.15$ & 22838.22 & 352.92 & 64.71 \\
NN cut $>0.20$ & 22133.21 & 344.70 & 64.20 \\
\hline
\end{tabular}

Table A.1. WS $D^{*}$ significance using the standard method and various neural network cuts. The maximum signal significance is at $\mathrm{NN}$ cut $>0.10$.

The maximum WS $D^{*}$ yield significance achieved with the neural network method yielded only $1.5 \%$ improvement over the standard method. When we started with lesser data, we got about 5 to $6 \%$ improvements over the standard method, but after adding all available data, the improvement dropped to $1.5 \%$. We ran the complete analysis to generate the ratio plots using the neural network classified data to see if the result looked sensible. The ratio plot was sensible and uncertainties on the values of the mixing parameters were slightly reduced as was expected. For a data sample of about $2.5 \mathrm{fb}^{-1}$, we compare the results of the standard method and the neural network analysis in Figure A.4.

\section{A.4 Some variables used as Neural network inputs}

Some of the input variables used for the neural network analysis are shown in Figures A.5 through A.15. In all the plots, the red data shows the distribution of the signal sample and the black data shows the distribution of the background sample. 

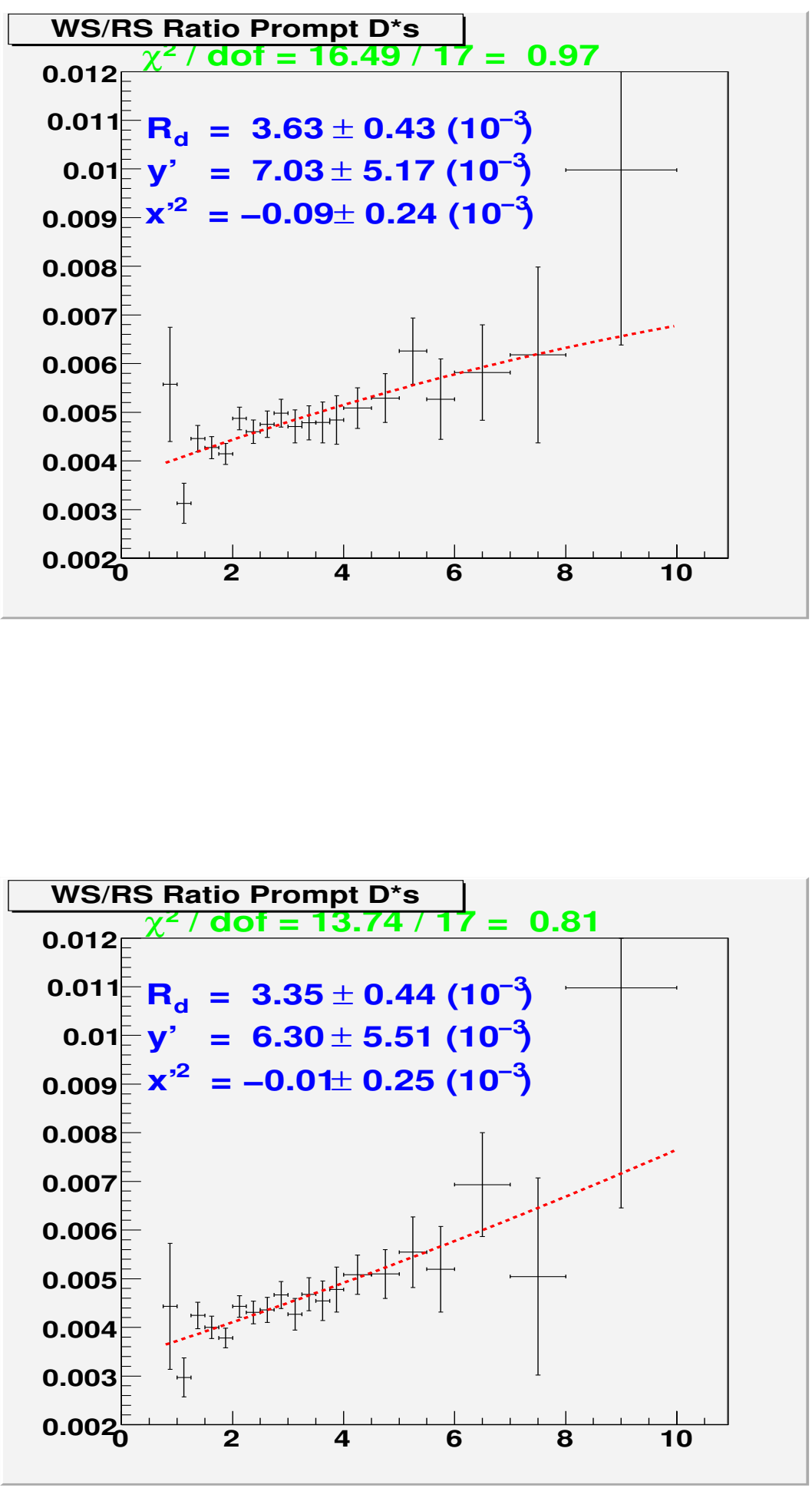

Figure A.4. Comparison of the ratio plots obtained from the neural network method (top) and from the standard analysis (bottom) for $2.5 \mathrm{fb}^{-1}$ data. 


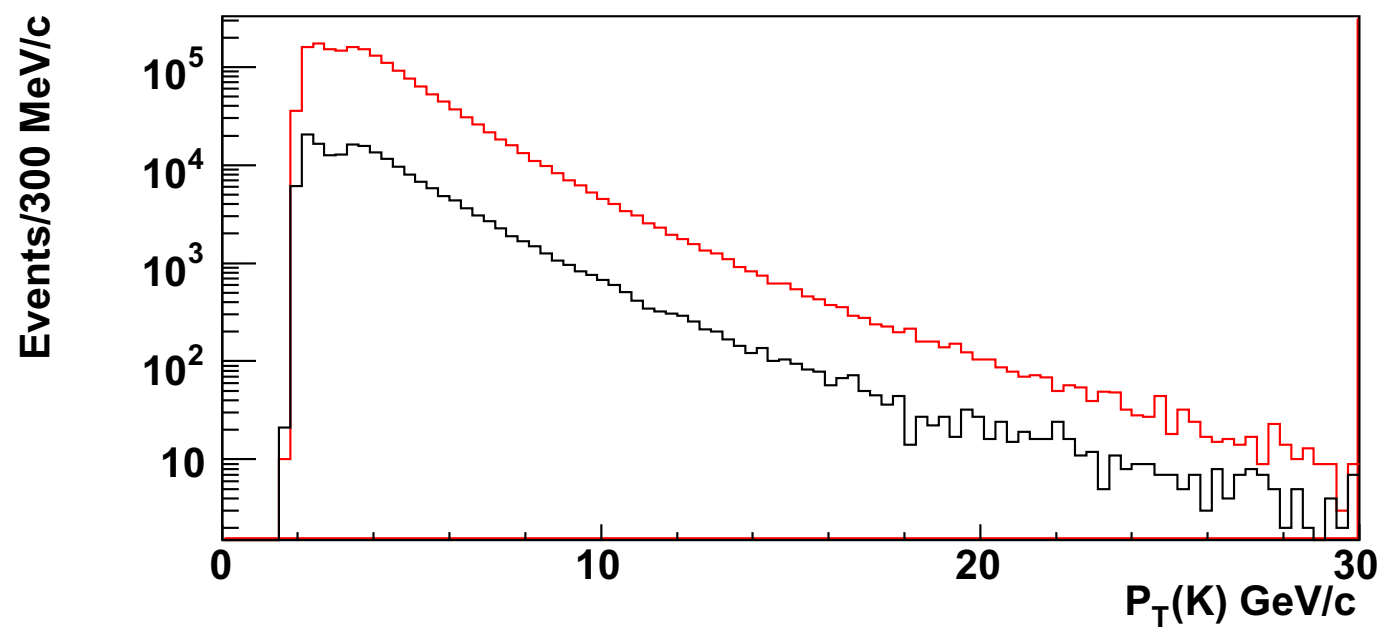

Figure A.5. Comparison of the transverse momentum of the kaon for signal and background samples.

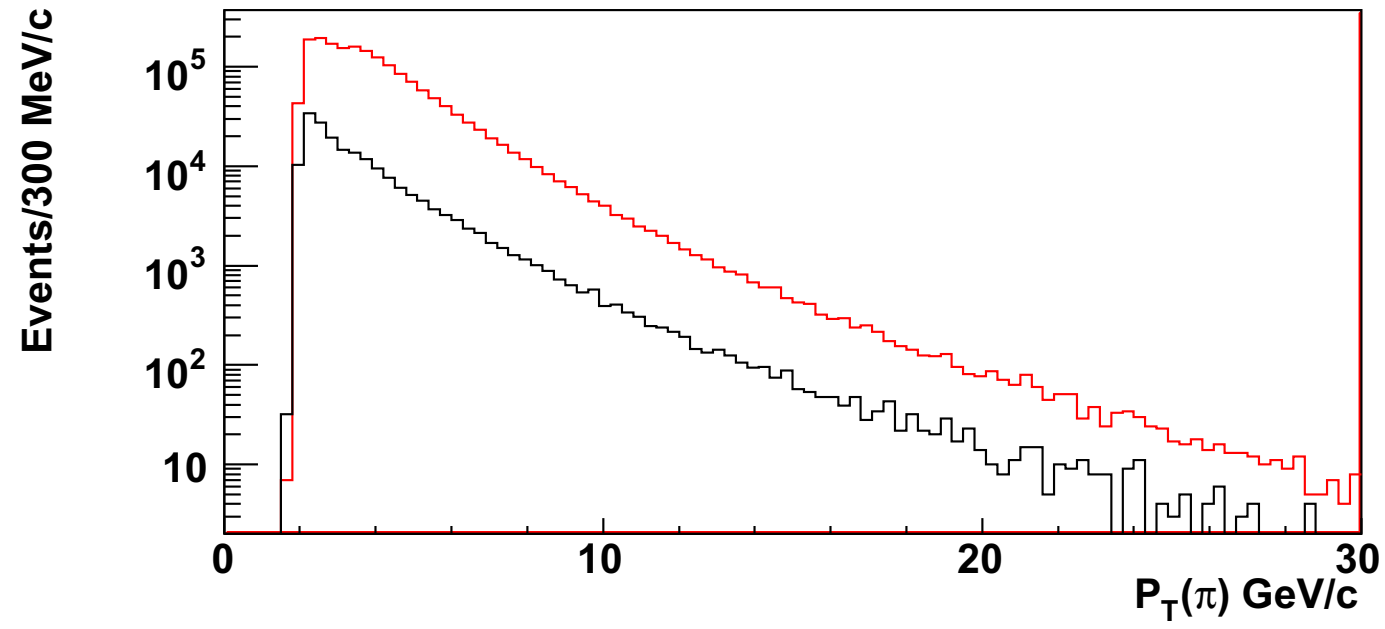

Figure A.6. Comparison of the transverse momentum of the pion for signal and background samples. 


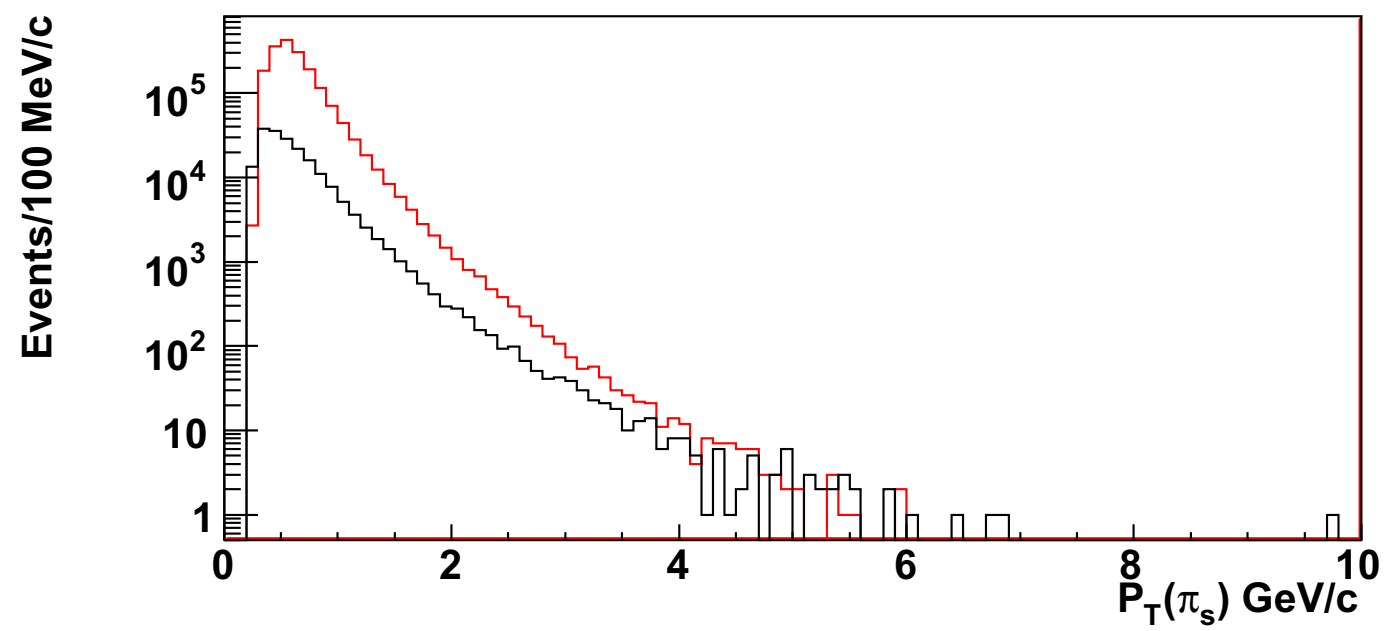

Figure A.7. Comparison of the transverse momentum of the soft pion for signal and background samples.

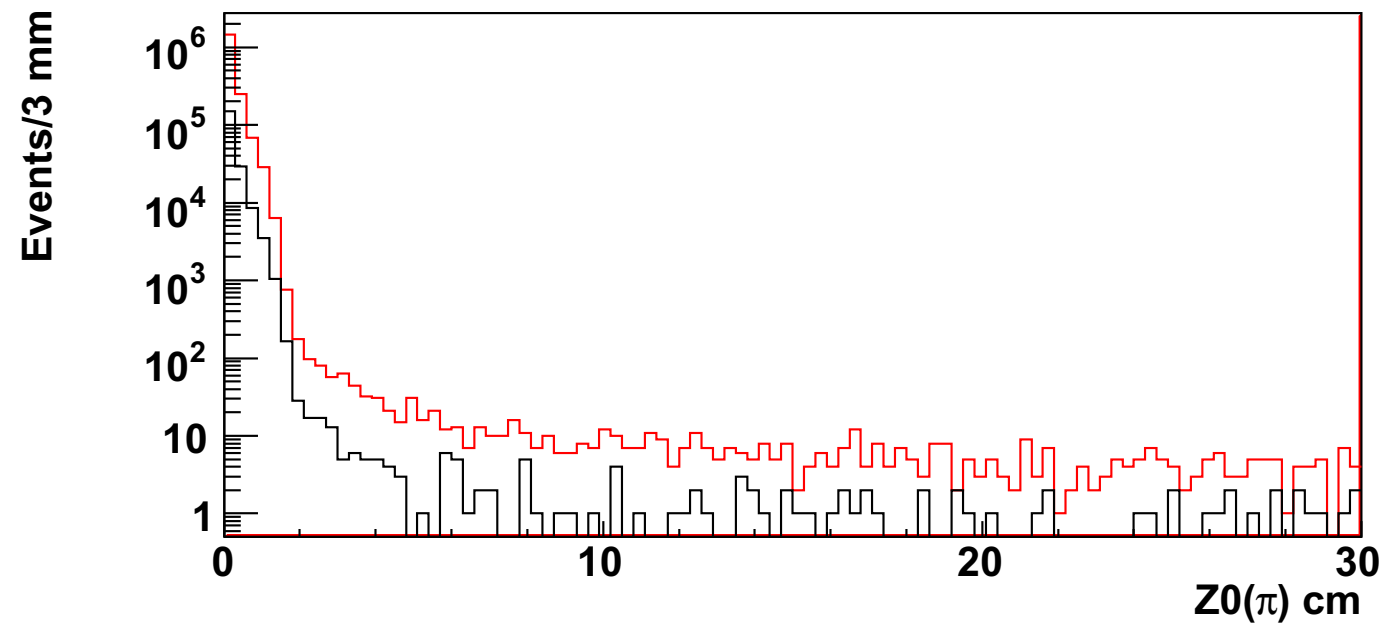

Figure A.8. Comparison of the Z0 variable-the point of closest approach along the beam line -for the stable pion for signal and background samples. 


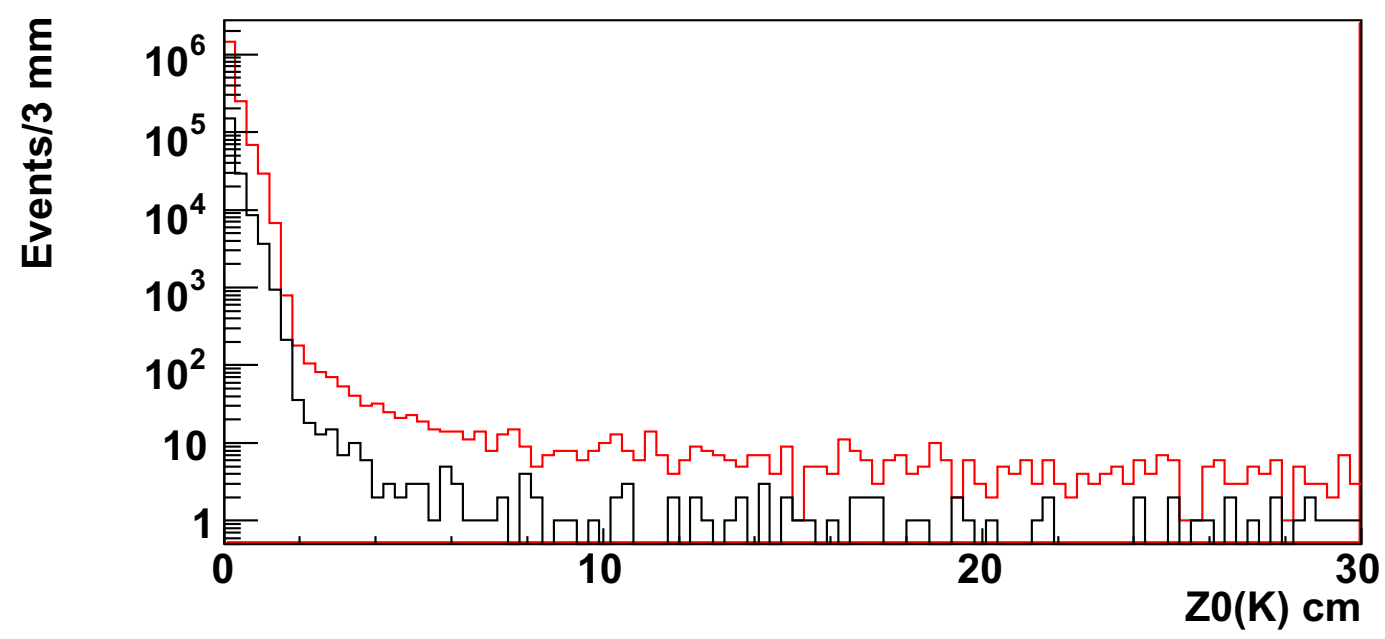

Figure A.9. Comparison of the $\mathrm{Z} 0$ variable-the point of closest approach along the beam line -for the kaon for signal and background samples.

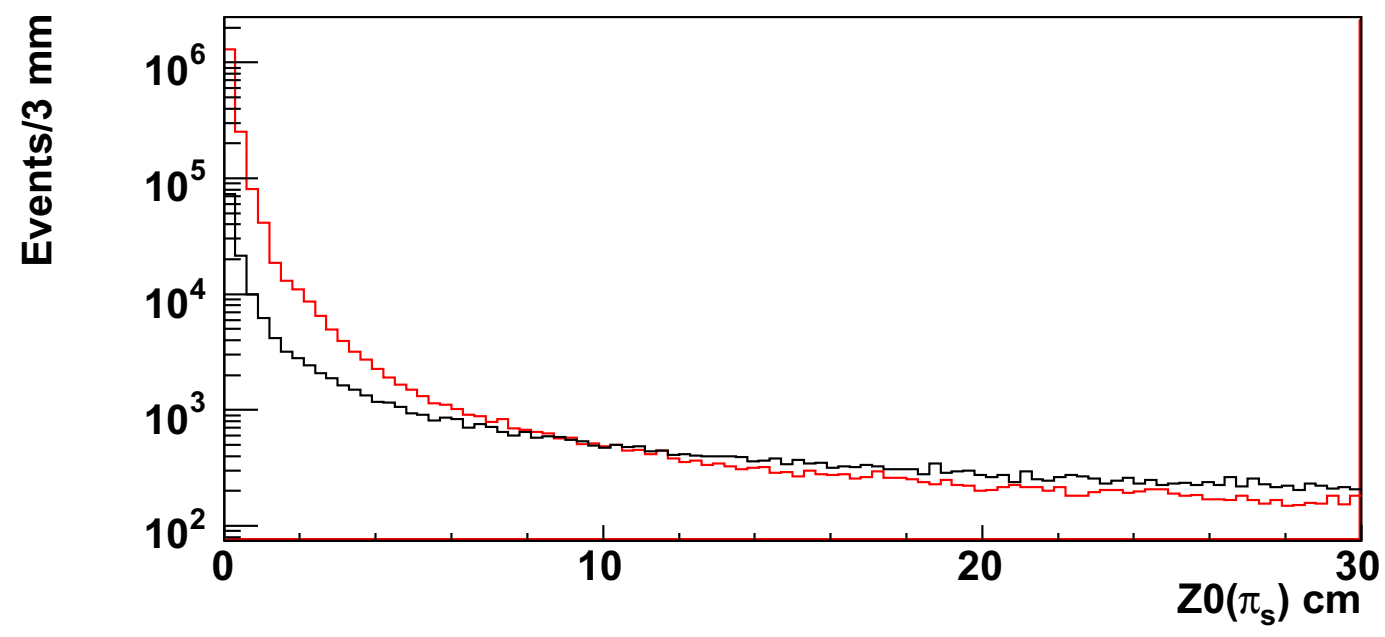

Figure A.10. Comparison of the $\mathrm{Z} 0$ variable-the point of closest approach along the beam line -for the soft pion for signal and background samples. 


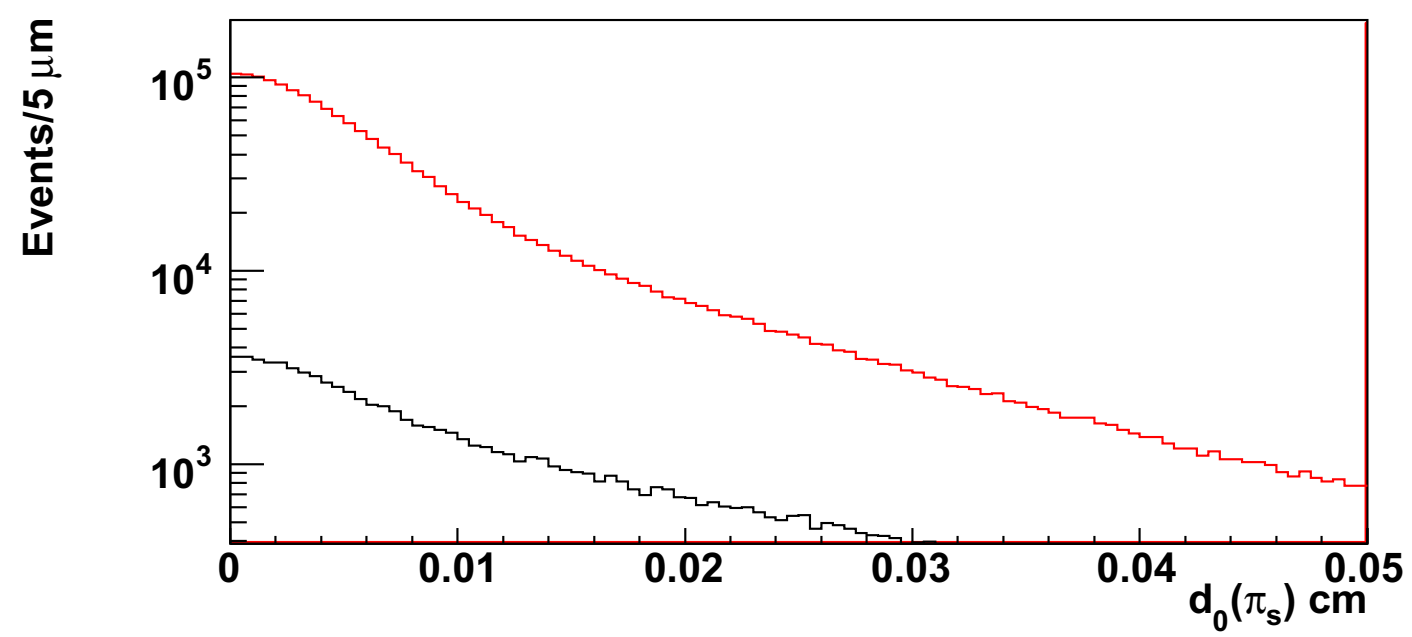

Figure A.11. Comparison of the impact parameter of the soft pion for signal and background samples.

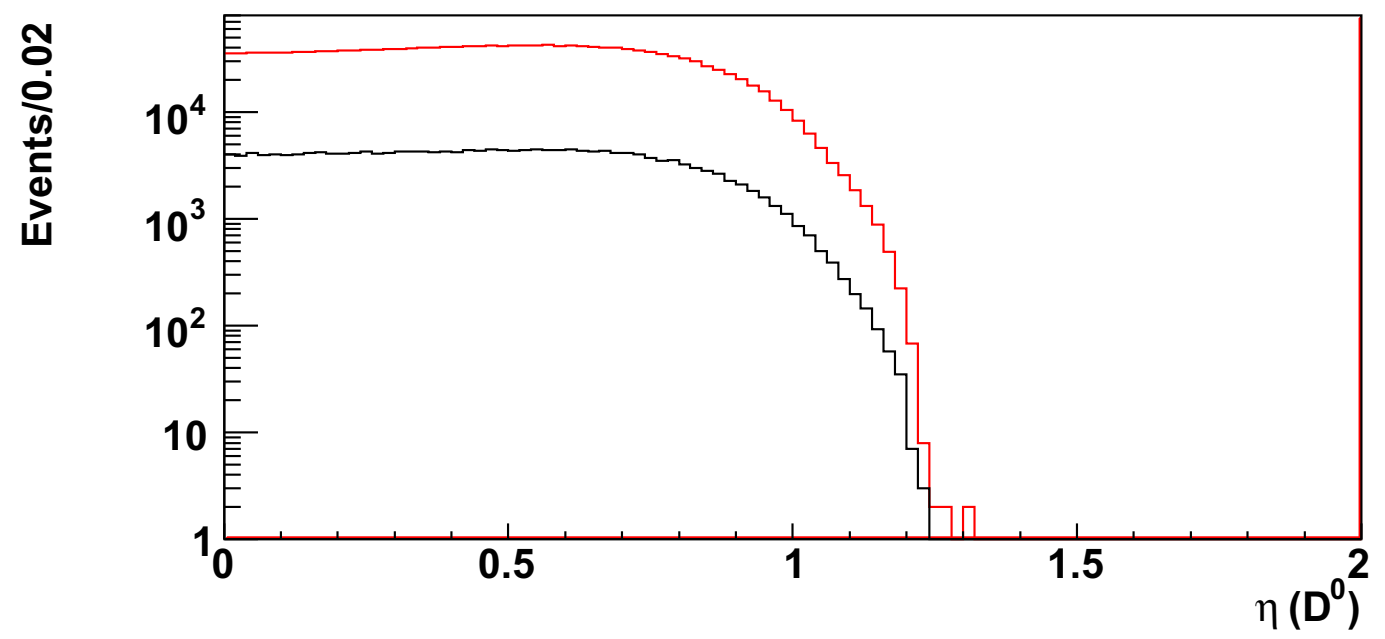

Figure A.12. Comparison of the $\eta$ variable of the $D^{0}$ for signal and background samples. 


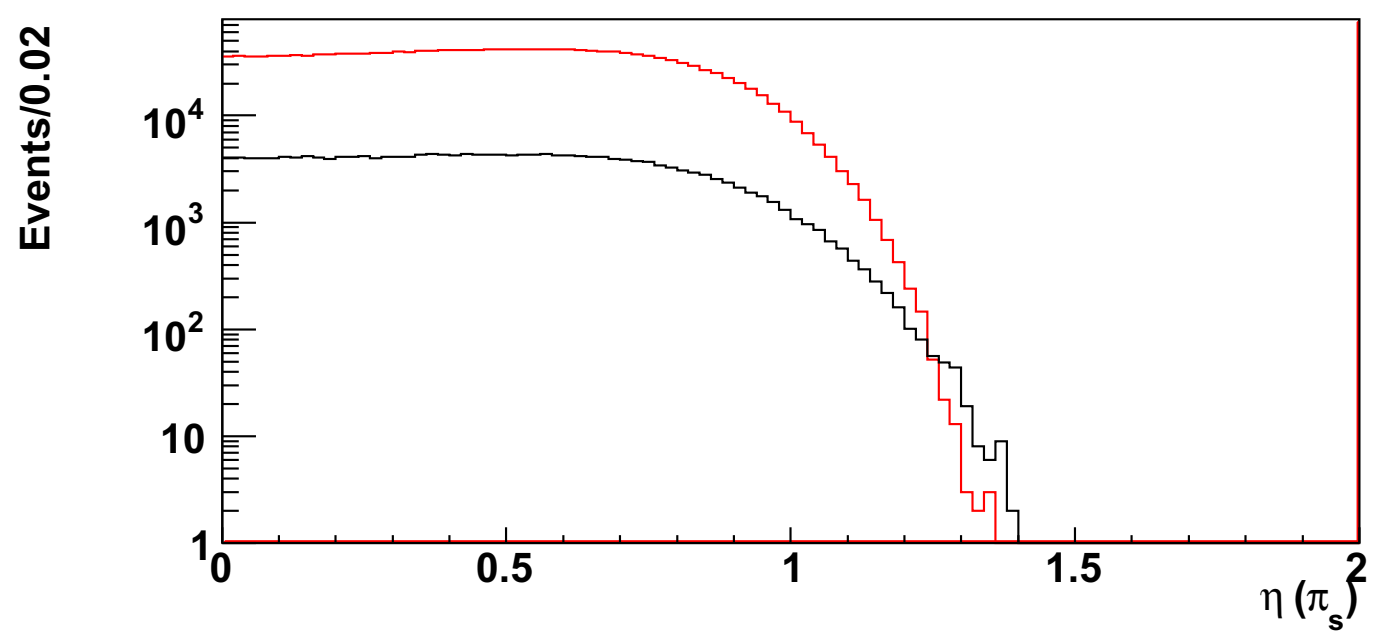

Figure A.13. Comparison of the $\eta$ variable of the soft pion for signal and background samples.

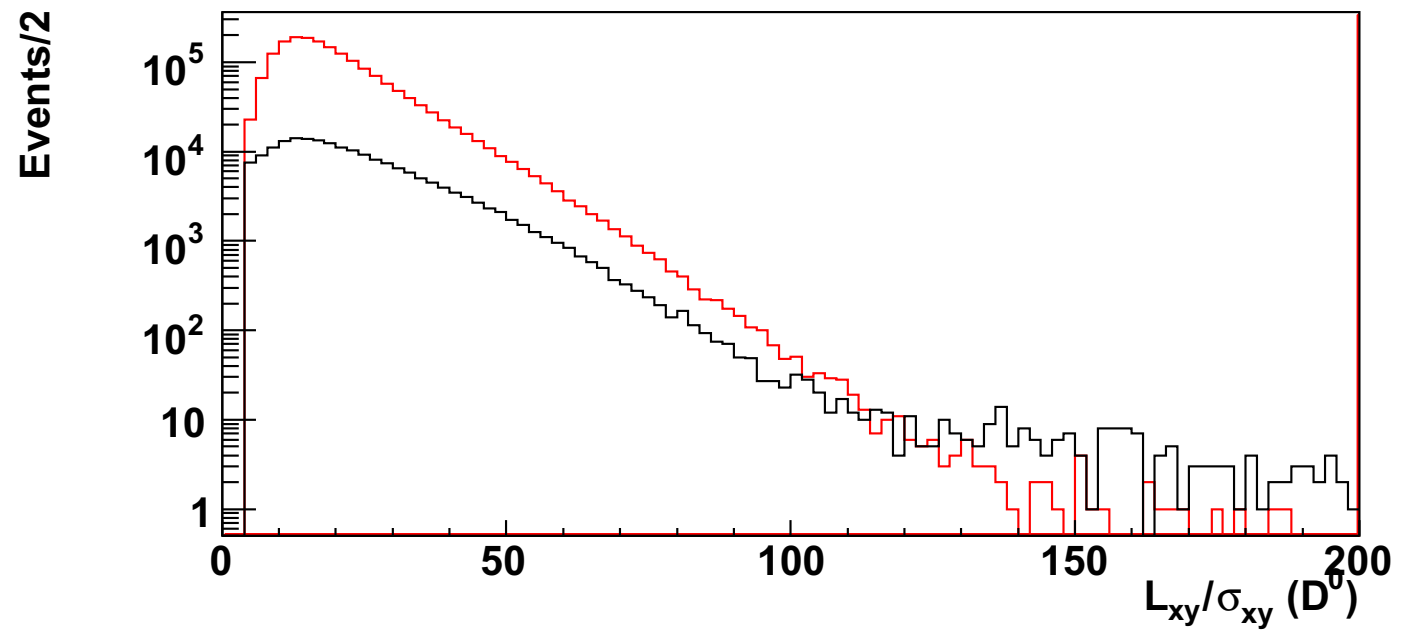

Figure A.14. Comparison of the transverse decay length significance of the $D^{0}$ for signal and background samples. 


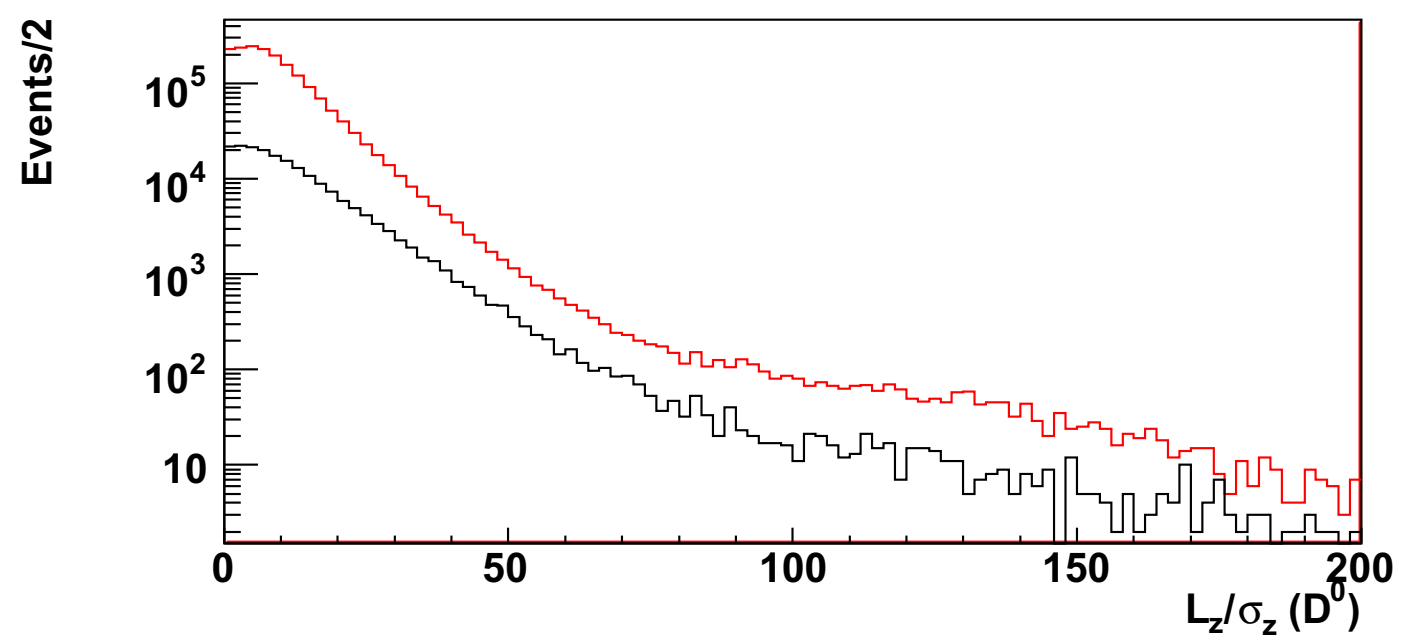

Figure A.15. Comparison of the $z$ component of the decay length significance of the $D^{0}$ for signal and background samples. 


\section{Appendix B}

\section{OTHER ATTEMPTS TO IMPROVE THE ANALYSIS}

We tried to improve the standard analysis method by adding new cuts and by finding ways to reduce backgrounds. Two such studies are mentioned here: the $D^{0}$ isolation cut and the multiple candidate background study.

\section{B.1 $D^{0}$ isolation variable cut}

We tried to add a new cut using the $D^{0}$ isolation variable, where the isolation variable is defined as

$$
I_{t}=\frac{P_{T}^{\text {cand }}}{P_{T}^{\text {cand }}+\sum_{\Delta R<0.4} P_{T}},
$$

where $I_{t}$ is the isolation of tracks, $P_{t}^{\text {cand }}$ is the transverse momentum of the candidate particle, $P_{T}$ is the transverse momentum of all other tracks except descendants of the candidate particle, and $\Delta R=\sqrt{\Delta \eta^{2}+\Delta \phi^{2}}$, where $\eta$ is the pseudo-rapidity and $\phi$ is the azimuthal angle in $\eta-\phi$ space of the CDF II detector. The $\Delta R$ variable can have values $0.4,0.7$, or 1.0. In our analysis, we used $\Delta R=0.4$.

For a fixed radius $\Delta R=0.4$, we computed the WS $D^{*}$ yield for 10 isolation cuts using the standard yield technique. Figure B.1 shows the WS $D^{*}$ yield/uncertainty, where the uncertainty comes from the statistical error on the first fit parameter of the $K \pi$ mass fit and is equivalent to $\sqrt{S+B}$. As we can see from the figure, the yield significance is almost flat. This indicates that the isolation variable is not very useful as a standard cut.

\section{B.2 Multiple candidate background}

We applied $\mathrm{dE} / \mathrm{dx}, K \pi$ mass, and $\Delta M$ cuts on a small fraction of the data. We processed the resulting 146000 events to look for events with multiple $D^{0}$ candidates. We found 4745 events having two candidates and 140 events with three candidates. No events were found with more than three candidates. We also observed that the multiple candidates differ only 


\section{WS $D^{*}$ significance vs iso cut}

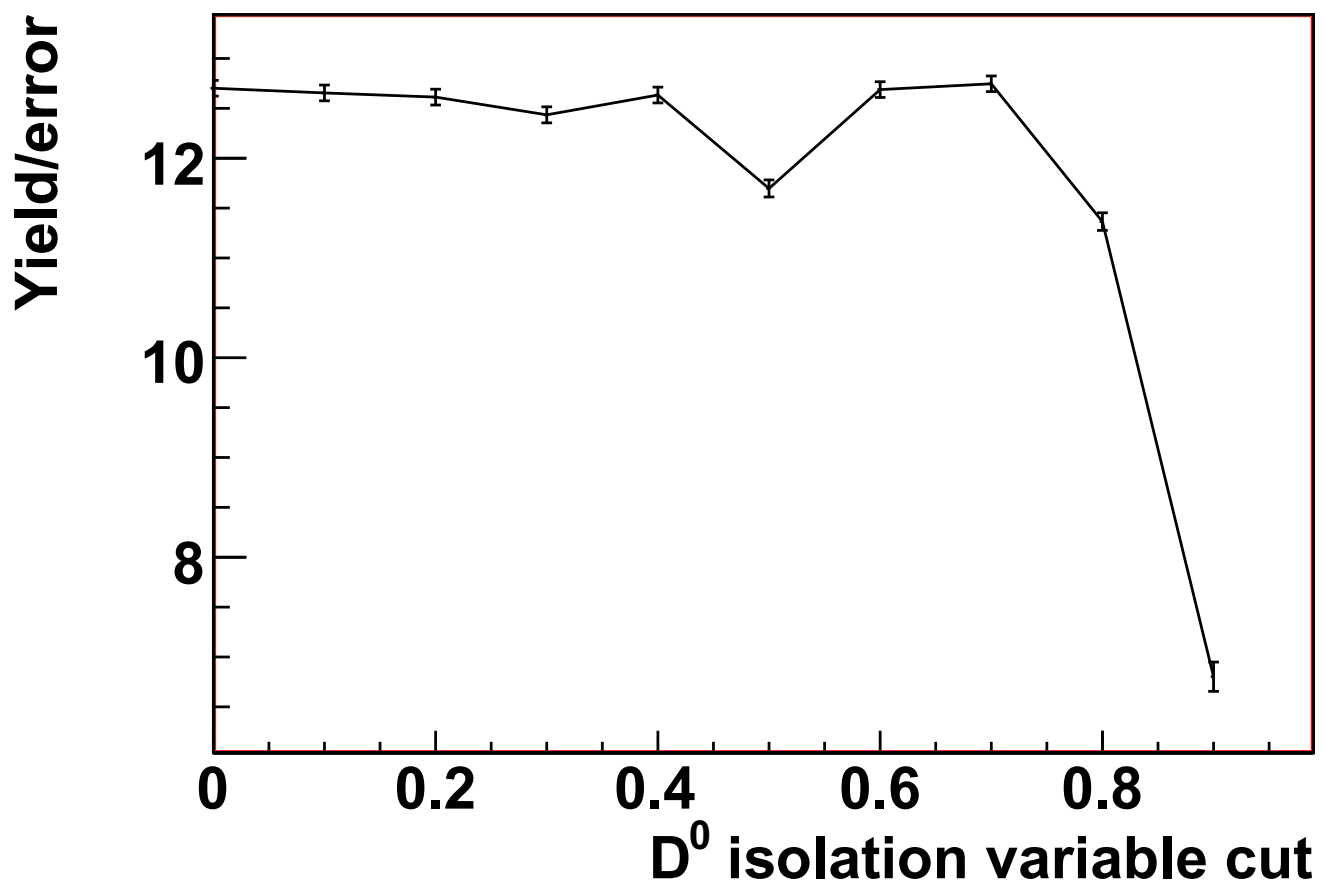

Figure B.1. WS $D^{*}$ yield vs. Isolation of $D^{0}$ cut. The yield is almost flat. It appears that the $D^{0}$ isolation variable is not useful as a standard cut.

in the selection of the soft pion with which they combine. In conclusion, only $3 \%$ of the total events have multiple candidates. Since this is a small number, we retain all the events in our data. 


\section{Appendix C}

\section{MONTE CARLO SIMULATION STUDIES}

This section describes some studies that were performed using Monte Carlo simulations.

\section{C.1 Monte Carlo Study of Impact Parameter Distribution of $D^{0}$ for Non- Prompt $D^{*} \mathbf{s}$}

In Section 3.6.4, we saw that the $D^{*}$ s originating from the primary vertex produce a narrow peak in the $D^{0}$ impact parameter (IP) distribution. The $D^{*}$ s originating from secondary decays produce a wide IP distribution. We assumed that these secondary decays are mostly $B$-decays. To qualitatively confirm this assumption, we applied the shapes of non-prompt IP distributions obtained from the CDF RS data to the simulated $B$ to charm distributions.

We produced a small sample of long lived $B$ to charm decays using the standard CDF realistic Monte Carlo. The MC events were generated using Pythia [48, 49]. The silicon vertex two track trigger was also simulated to select the events.

In a typical simulated event, two gluons from a $p-\bar{p}$ collision produce a $b-\bar{b}$ pair. The $b$ quarks combine with other quarks or anti-quarks to produce $B$ mesons. The $B$ mesons then decay into charm mesons. We included all long lived charm mesons such as $D^{0}, \Lambda_{c}^{+}$, $\Lambda_{c}^{-}, \Xi_{c}^{+}, \Xi_{c}^{0}, \Xi_{c c}^{++}$, and $\Omega_{c}^{0}$. We also included long lived $b$-baryons such as $\Lambda_{b}^{0}, \Sigma_{b}^{+}, \Sigma_{b}^{-}, \Sigma_{b}^{0}$, $\Xi_{b}^{-}, \Xi_{b}^{0}$, and $\Omega_{b}^{-}$. We treated all these decays as non-prompt background.

The Pythia output contains the information about the decay vertices, momentum, and energy of the simulated particles. From this information, we computed the impact parameter of the $D^{0}$ candidate using Equation 3.4. The required vectors $\mathbf{r}$ and $\mathbf{P}_{\mathbf{T}}$ were obtained from the $x, y$, and $z$ coordinates of the $D^{0}$ decay vertex and the associated momentum vector components $p_{x}, p_{y}$, and $p_{z}$. We computed the proper decay time of the $D^{0}$ from Equation 3.3.

We divided the data into 8 time bins. For each time bin, we fit the impact parameter distribution with a single Gaussian function. The mean of the Gaussian was fixed to zero, 


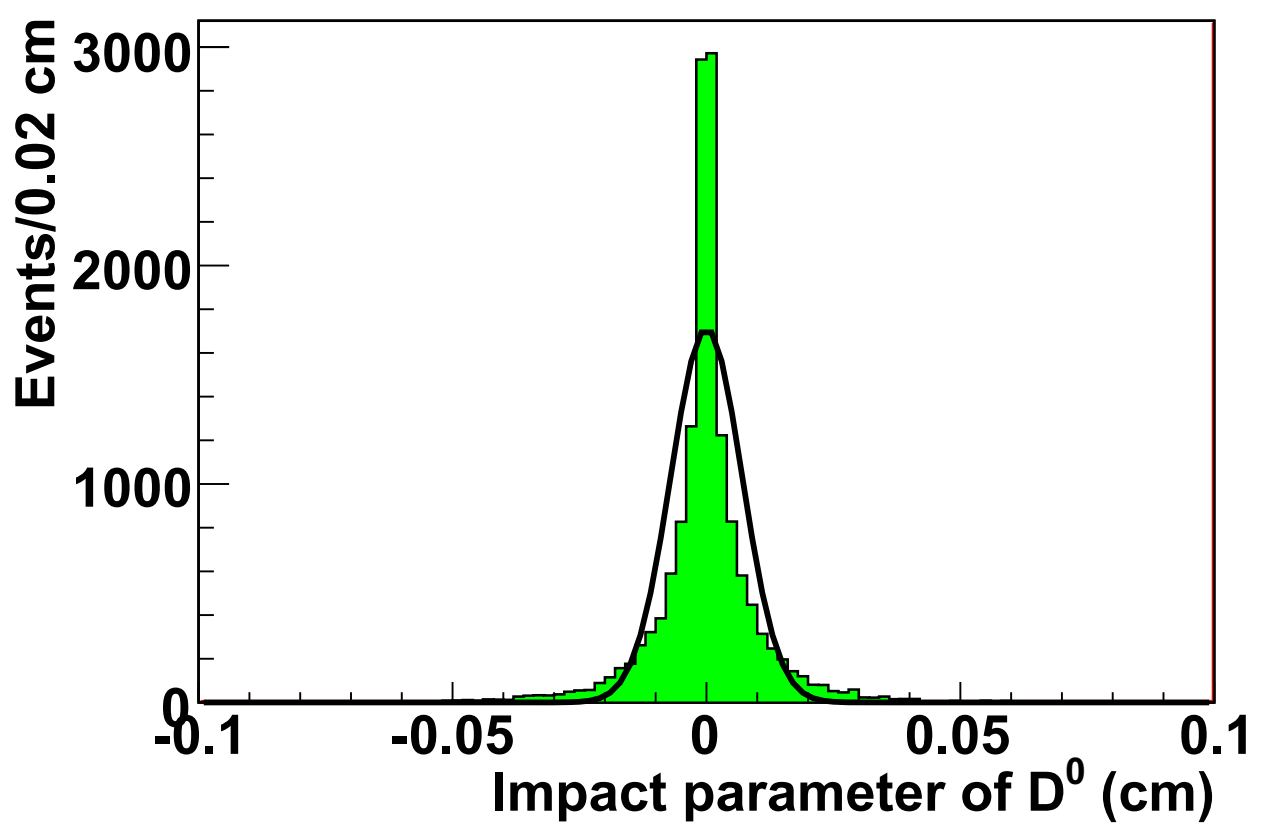

Figure C.1. IP distribution for simulated $B$ to charm decays. Time bin 1 (1.00 to $2.75 t / \tau$ ).

the amplitude was allowed to float, and the width of the Gaussian was fixed from the parametrization obtained from the CDF RS data, as described in Section 3.6.4. Figures C.1 through C.8 show these fits. These figures show that the non-prompt single Gaussian shapes obtained from the data qualitatively describe the Monte Carlo generated IP distributions. This supports our assumption that the secondary decays are $B$-decays.

\section{C.2 Lump study}

We tried to search for the decay that can cause the extra lump in the RS $K \pi$ background, discussed in Section 3.7.2. We generated a realistic Monte Carlo simulation of the semileptonic decay chain $D^{*} \rightarrow D^{0} \pi^{+} \rightarrow K^{-} \mu^{+} \nu_{\mu}$. For a quick study, we generated 3000 events of this decay chain using the Pythia generator. We found that the $D^{0}$ mass reconstructed from this decay falls below $1.8 \mathrm{GeV} / c^{2}$ and after applying the $D^{*}$ mass difference cut (but not all the standard cuts), it cannot form a peak at $1.83 \mathrm{GeV} / c^{2}$ where the lump was found in the real RS $D^{0}$ data. Figure C.9 shows the invariant mass of the $D^{0}$ reconstructed from 


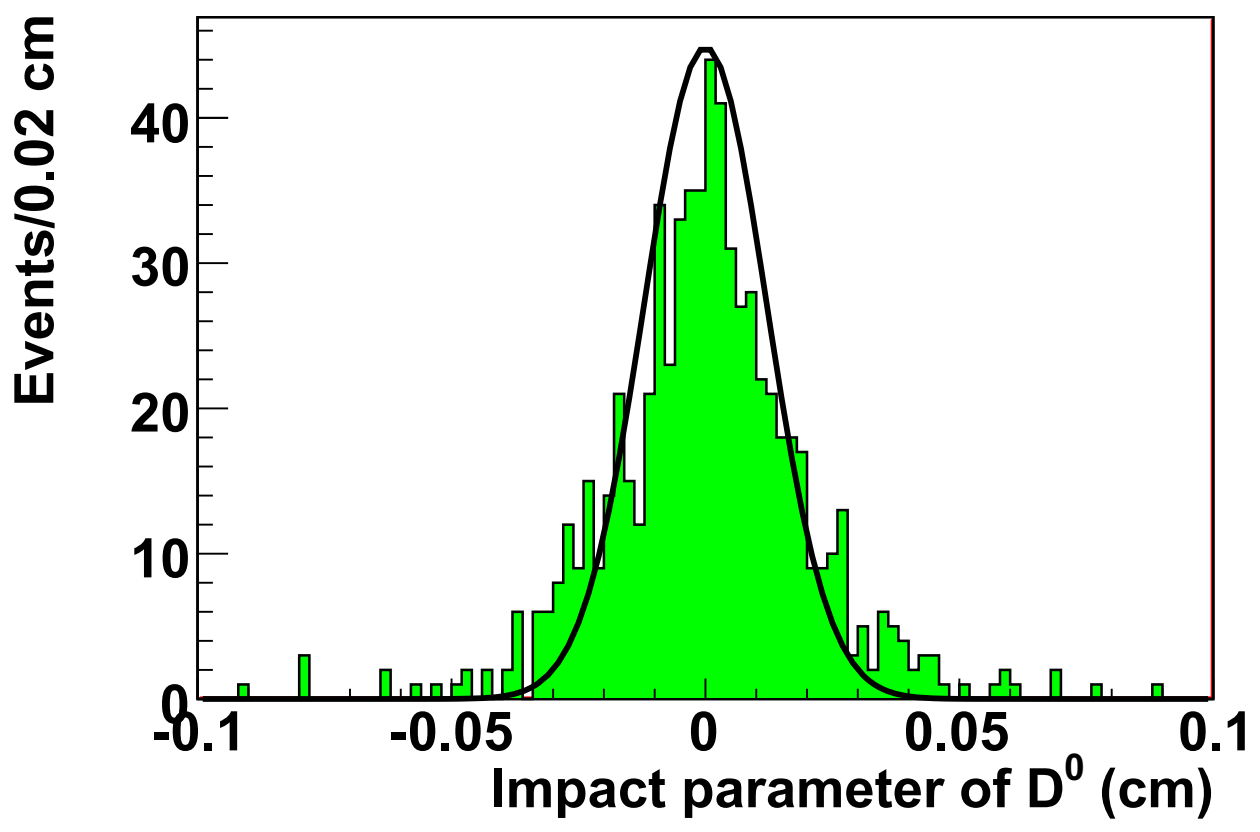

Figure C.2. IP distribution for simulated $B$ to charm decays. Time bin $2(2.75$ to $3.00 t / \tau)$.

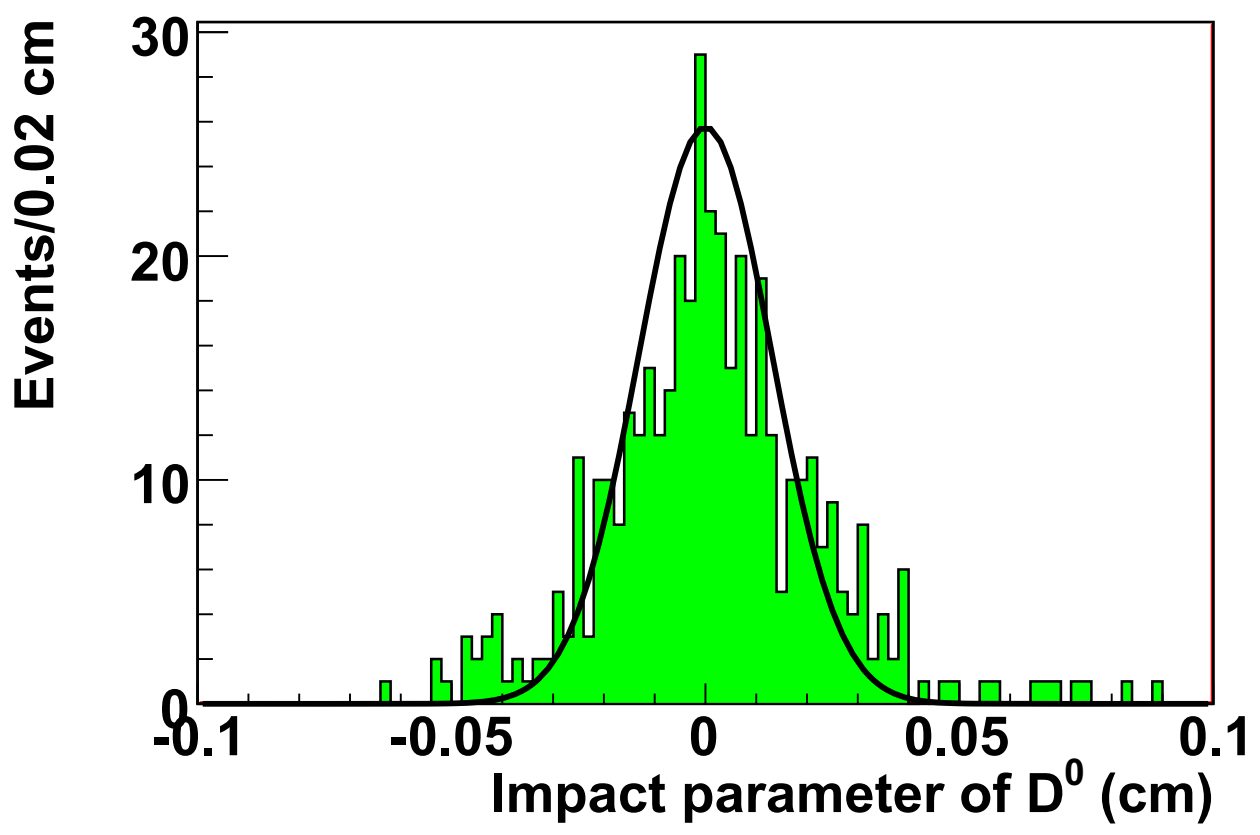

Figure C.3. IP distribution for simulated $B$ to charm decays. Time bin 3 (3.00 to $3.5 t / \tau)$. 


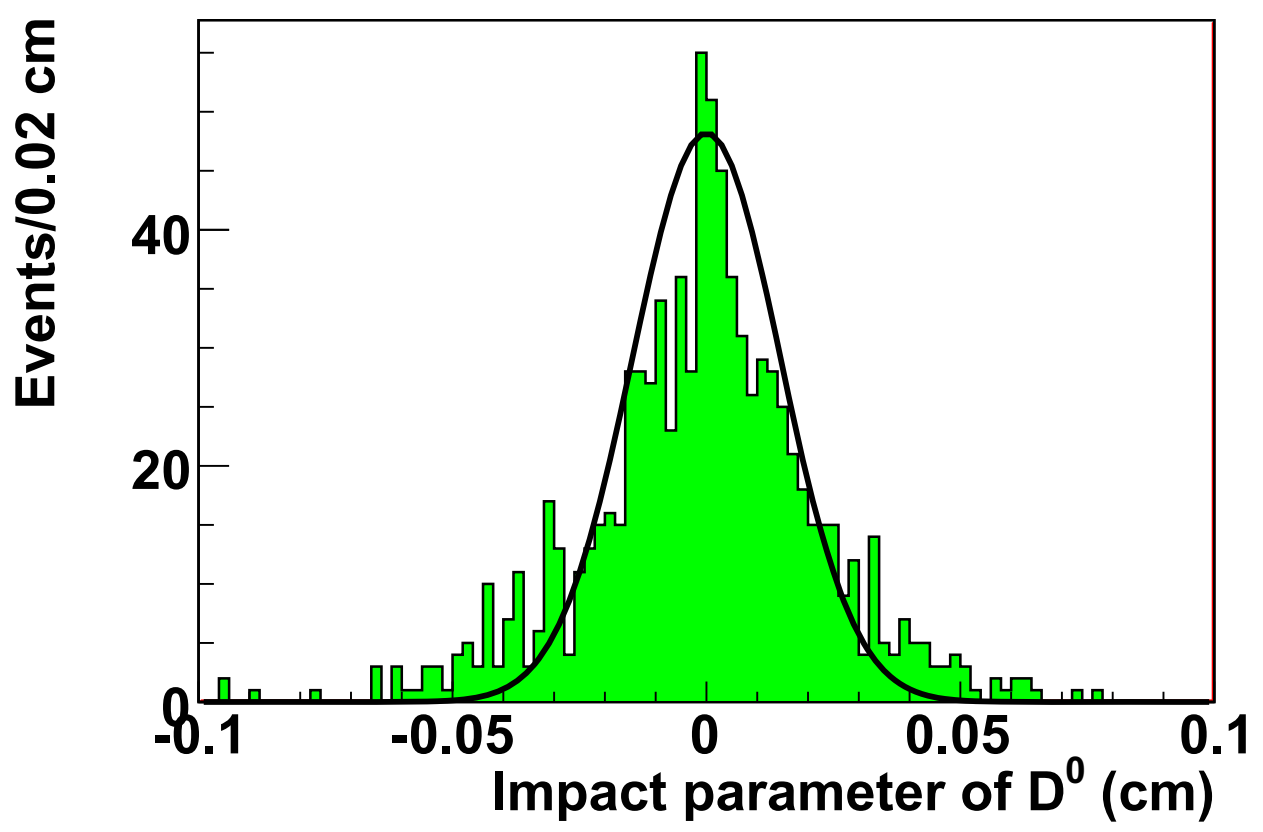

Figure C.4. IP distribution for simulated $B$ to charm decays. Time bin 4 (3.5 to $4.0 t / \tau)$.

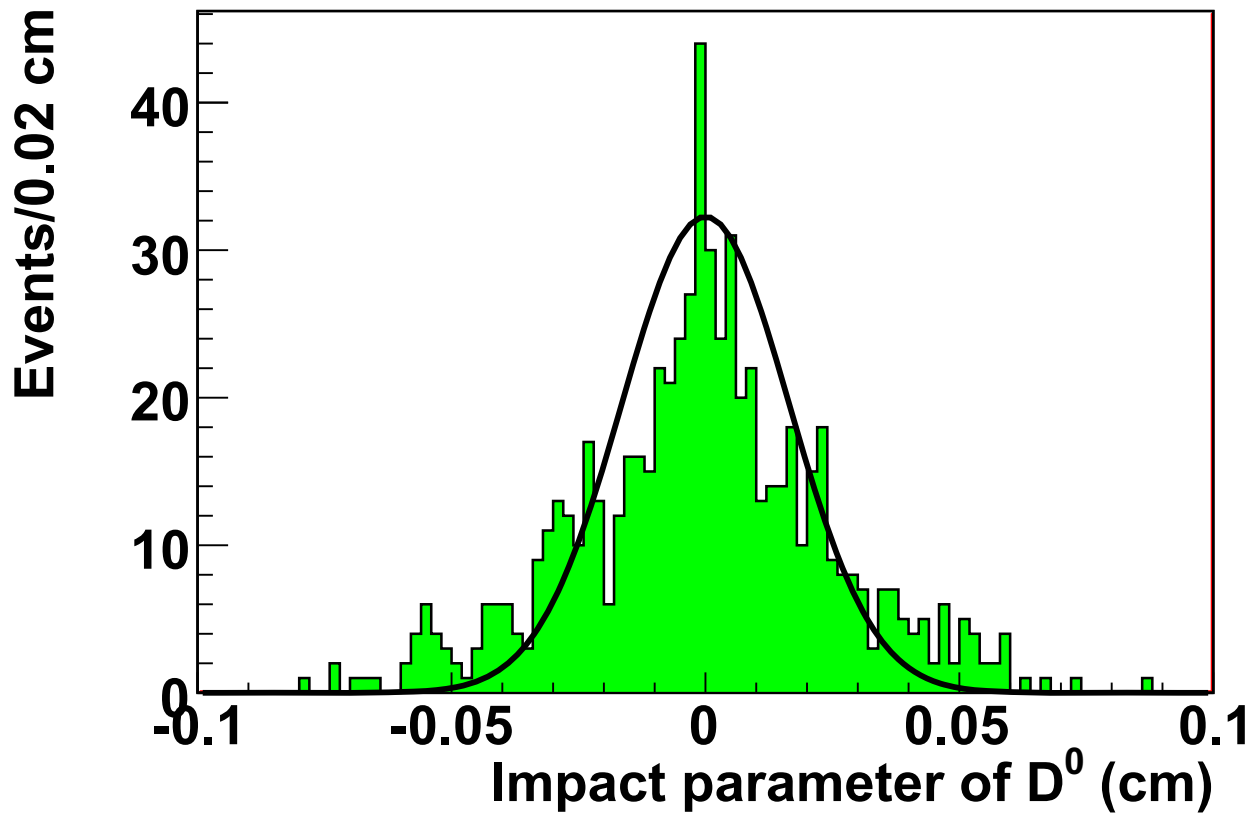

Figure C.5. IP distribution for simulated $B$ to charm decays. Time bin 5 (4.00 to $5.5 t / \tau)$. 


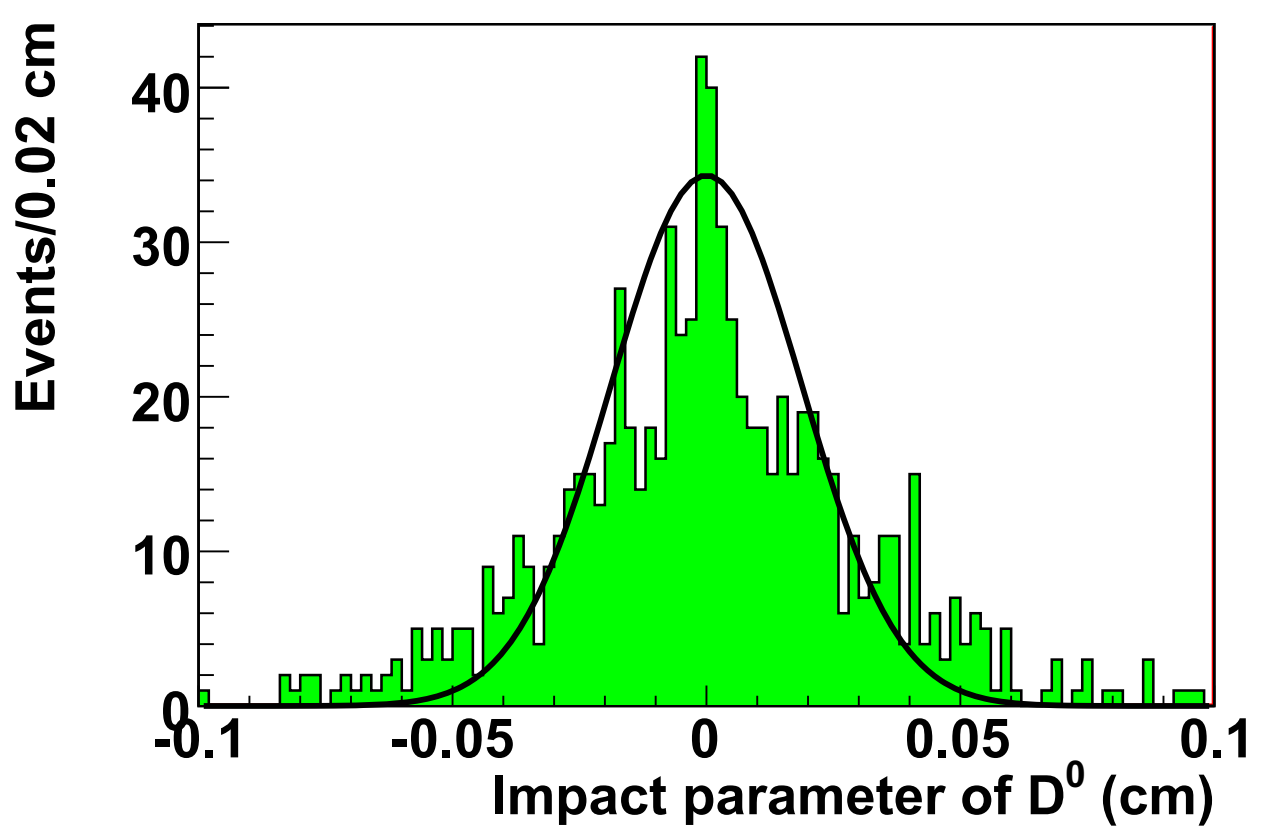

Figure C.6. IP distribution for simulated $B$ to charm decays. Time bin 6 (5.5 to $7.0 t / \tau)$.

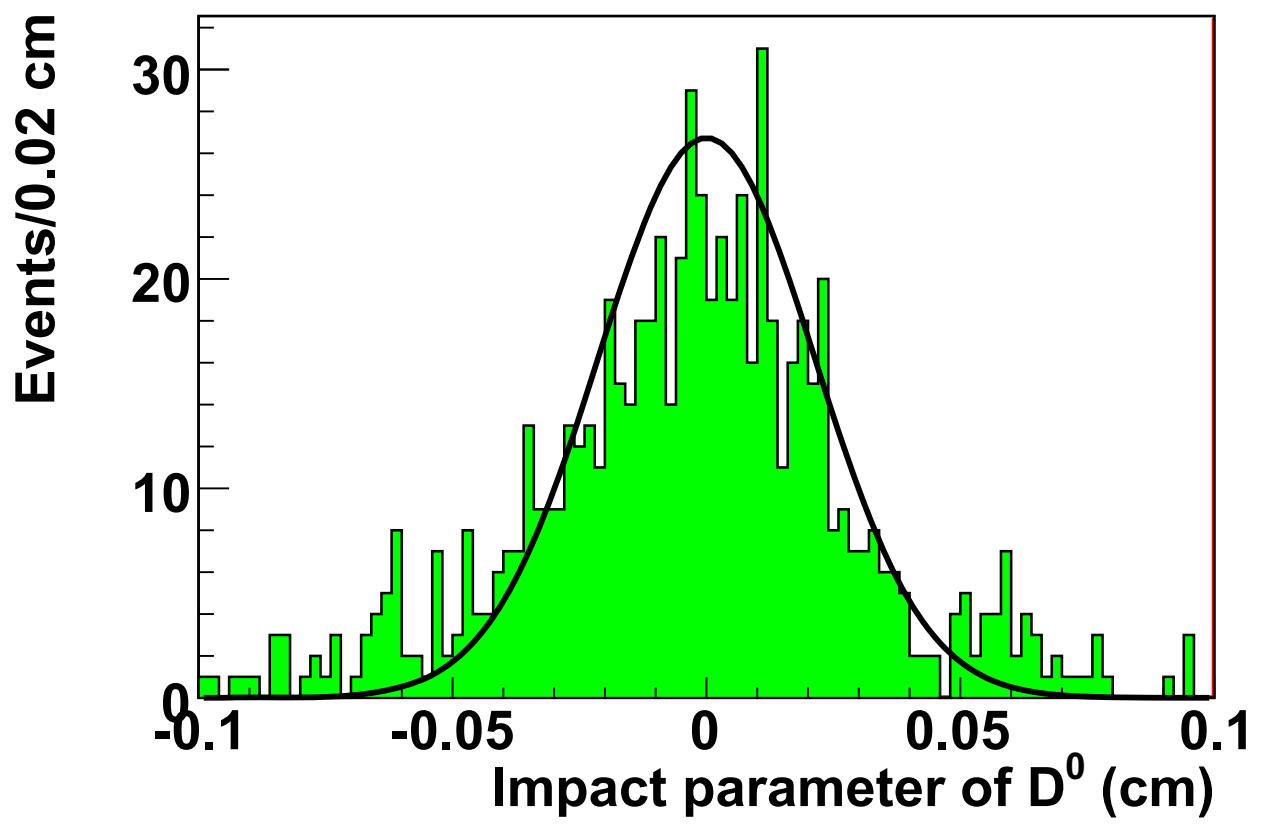

Figure C.7. IP distribution for simulated $B$ to charm decays. Time bin 7 (7.00 to $8.00 t / \tau)$. 


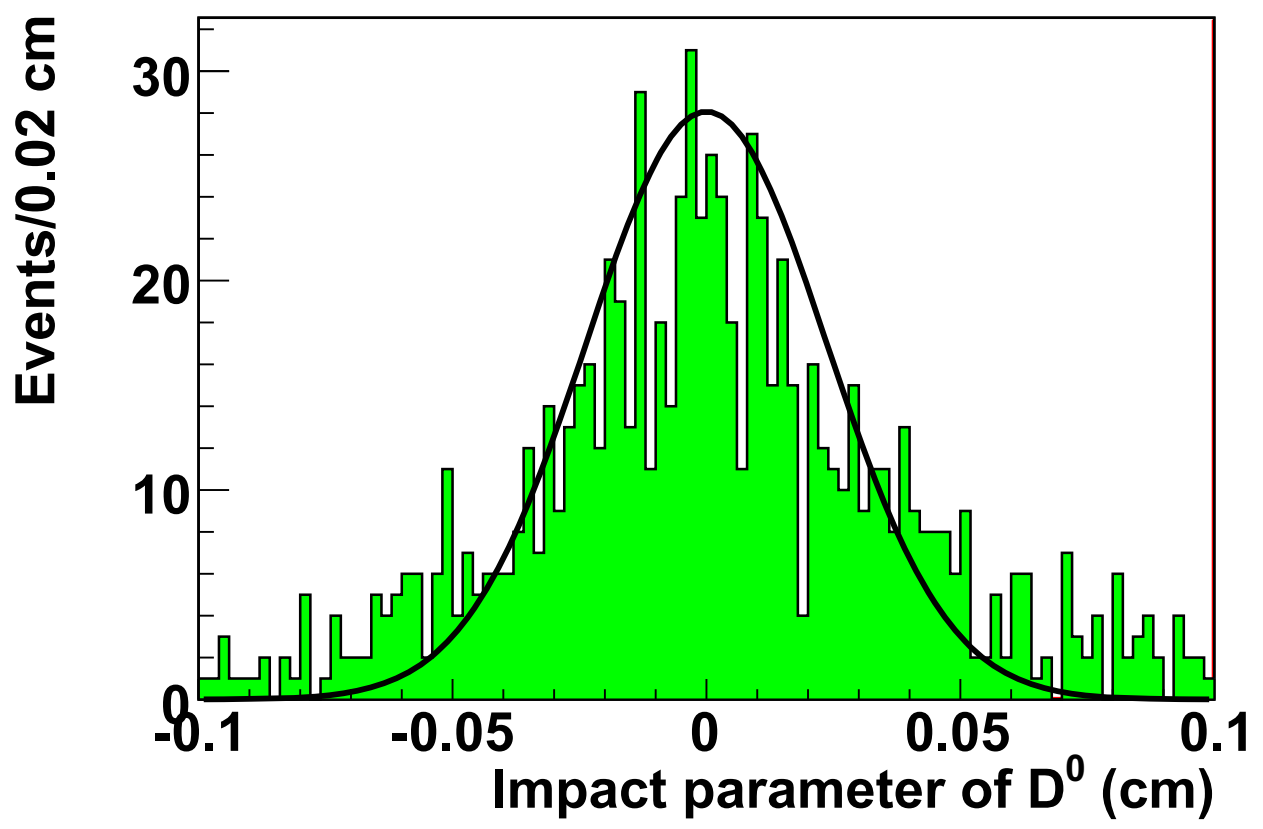

Figure C.8. IP distribution for simulated $B$ to charm decays. Time bin 8 (8.00 to 10.00 $t / \tau)$.

the $D^{0} \rightarrow K^{-} \mu^{+} \nu_{\mu}$ decay.

Similar results were found for other decay chains that were tried. Figure C.10 shows the invariant mass of $\Lambda_{c}^{+}$reconstructed from $\Lambda_{c}^{+} \rightarrow p^{+} K^{-} \pi^{+}$decay. This was one of the candidates that was expected to cause the RS lump. We also tried $D_{s}^{+} \rightarrow K K \pi$ and reconstructed a $D^{0}$ from the oppositely charged kaon and pion. Another attempt was $D_{s} \rightarrow \pi \pi \pi$ decay, with one of the pions reconstructed as a kaon. None of these decays had their mean invariant mass close to $1.83 \mathrm{GeV} / c^{2}$. The cause of the extra lump remains a mystery. 


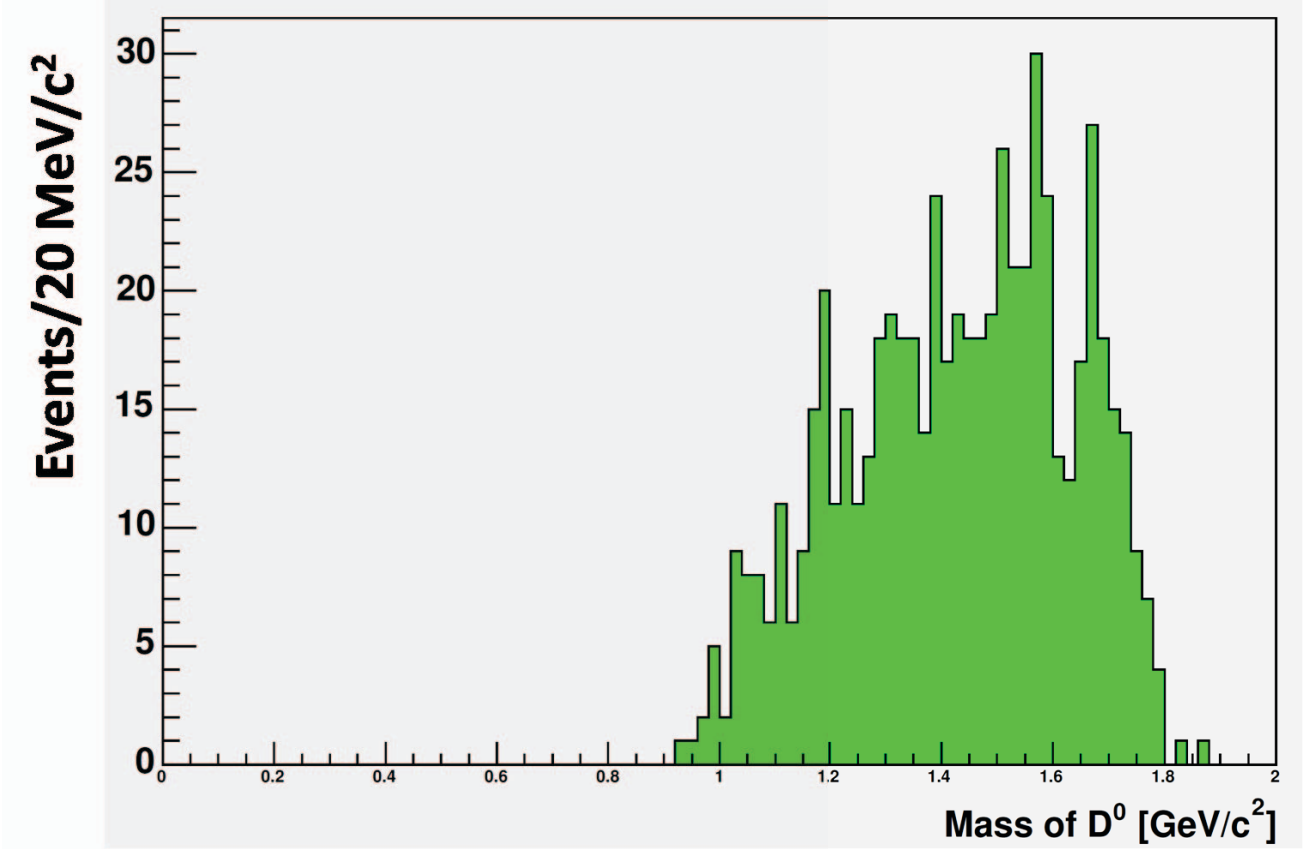

Figure C.9. The $D^{0}$ mass distribution for the $D^{0}$ reconstructed from simulated $D^{0} \rightarrow$ $K^{-} \mu^{+} \nu_{\mu}$ decay assuming the muon as a pion. We applied the standard mass difference cut. The mean invariant mass falls below $1.83 \mathrm{GeV} / c^{2}$. The lump in RS $\left.K\right] p i$ mass plot was found at $1.83 \mathrm{GeV} / c^{2}$. 


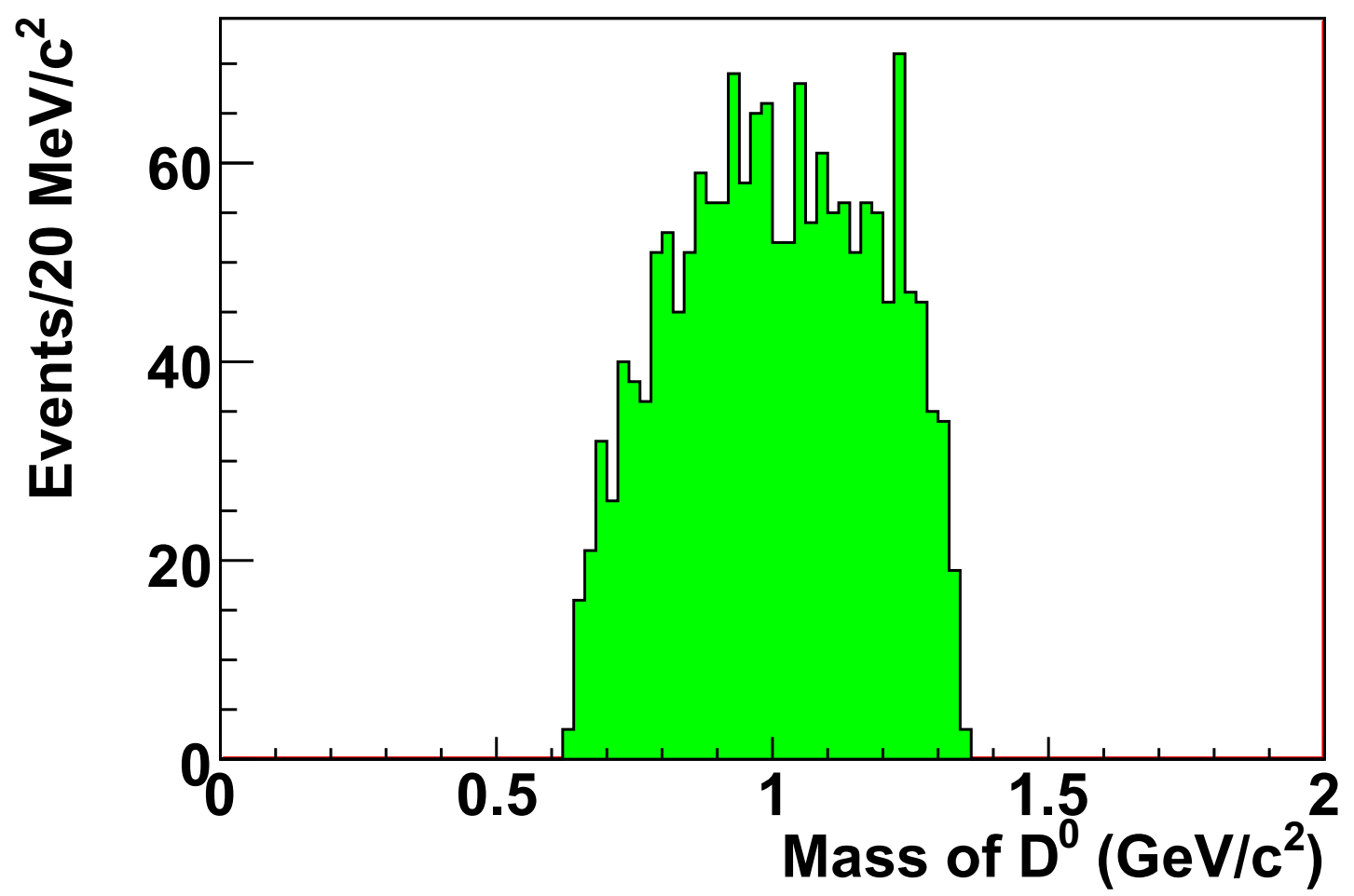

Figure C.10. Invariant mass of $\Lambda_{c}^{+}$reconstructed from simulated $\Lambda_{c}^{+} \rightarrow p^{+} K^{-} \pi^{+}$decay. This was another decay that we thought would cause the lump in RS plot. But the mean invariant mass of $\Lambda_{c}^{+}$found to be well below $1.83 \mathrm{GeV} / c^{2}$. 


\section{REFERENCES}

[1] C. Amsler et al. [Particle Data Group], "Review of particle physics," Phys. Lett. B 667, 1 (2008).

[2] C. Amsler et al. [Particle Data Group], "Review of particle physics," "Particle Listings: Charmed Mesons $\left(D^{0}\right), "$ Phys. Lett. B 667, 1 (2008).

[3] M. G. Schmidt, "Beyond the standard model," Lect. Notes, Phys. 721, 51 (2007).

[4] J. Nachtman [CDF and D0 Collaborations], "Collider searches for physics beyond the standard model," Acta Phys. Polon. Supp. 1, 425 (2008).

[5] E. Golowich, J. Hewett, S. Pakvasa and A. A. Petrov, "Implications of $D^{0}-\bar{D}^{0}$ Mixing for New Physics," Phys. Rev. D 76, 095009 (2007).

[6] E. Golowich and A. A. Petrov, "Short distance analysis of D0 - anti-D0 mixing," Phys. Lett. B 625, 53 (2005).

[7] A. F. Falk, Y. Grossman, Z. Ligeti and A. A. Petrov, "SU(3) breaking and D0 - anti-D0 mixing," Phys. Rev. D 65, 054034 (2002).

[8] C. Amsler et al. [Particle Data Group], "Review of particle physics," " $D^{0}-\overline{D^{0}}$ Mixing," Phys. Lett. B 667, 1 (2008).

[9] E. Golowich, S. Pakvasa and A. A. Petrov, "New physics contributions to the lifetime difference in D0 - anti-D0 mixing," Phys. Rev. Lett. 98, 181801 (2007).

[10] A. A. Petrov, "Charm mixing in the Standard Model and beyond," Int. J. Mod. Phys. A 21, 5686 (2006).

[11] E. Golowich, J. Hewett, S. Pakvasa and A. A. Petrov, "Relating $D^{0}-\bar{D}^{0}$ Mixing and $D^{0} \rightarrow l^{+} l^{-}$with New Physics," Phys. Rev. D 79, 114030 (2009).

[12] C. Amsler et al. [Particle Data Group], "Review of particle physics," "CP Violation in Meson Decays," Phys. Lett. B 667, 1 (2008). 
[13] C. Amsler et al. [Particle Data Group], "Review of particle physics," "The CKM Quark Mixing Matrix," Phys. Lett. B 667, 1 (2008).

[14] A. A. Petrov, "Constraining New Physics from D0-anti-D0 mixing," arXiv:0806.2498 [hep-ph].

[15] A. A. Petrov, "D0 anti-D0 mixing," arXiv:hep-ph/0207212.

[16] L. M. Zhang et al. [BELLE Collaboration], "Improved constraints on D0-anti-D0 mixing in $D^{0} \rightarrow K^{+} \pi^{-}$decays at Belle," Phys. Rev. Lett. 96, 151801 (2006).

[17] L. Wolfenstein, "Parametrization Of The Kobayashi-Maskawa Matrix," Phys. Rev. Lett. 51, 1945 (1983).

[18] K. Flood, "Evidence for $D^{0}-\bar{D}^{0}$ Mixing at Babar," arXiv:0705.2785 [hep-ex].

[19] B. Aubert et al. [BABAR Collaboration], "Evidence for $D^{0}$-anti-D0 Mixing," Phys. Rev. Lett. 98, 211802 (2007).

[20] M. Staric et al. [Belle Collaboration], "Evidence for $D^{0}-\bar{D}^{0}$ Mixing," Phys. Rev. Lett. 98, $211803(2007)$.

[21] D. Son et al. "Lepton and Photon Interactions at High Energy," Kyungpook National University Press (2009), ISBN: 978-89-7180-229-8.

[22] T. Aaltonen et al. [CDF Collaboration], "Evidence for $D^{0}-\bar{D}^{0}$ mixing using the CDF II Detector," Phys. Rev. Lett. 100, 121802 (2008).

[23] B. Aubert et al. [BABAR Collaboration], "Measurement of $D^{0}-\bar{D}^{0}$ mixing using the ratio of lifetimes for the decays $D^{0} \rightarrow K^{-} \pi^{+}, K^{-} K^{+}$, and $\pi^{-} \pi^{+}, "$ Phys. Rev. D 78, 011105 (2008).

[24] U. Bitenc et al. [BELLE Collaboration], "Improved search for $D^{0}$ mixing using semileptonic decays at Belle," Phys. Rev. D 77, 112003 (2008).

[25] R. Andreassen [BaBar Collaboration], " $D^{0}-\bar{D}^{0}$ Mixing Analyses at BaBar," arXiv:0804.0020 [hep-ex]. 
[26] K. Abe et al. [BELLE Collaboration], "Measurement of D0-D0bar mixing in $D^{0} \rightarrow$ $K_{s} \pi^{+} \pi^{-}$decays," Phys. Rev. Lett. 99, 131803 (2007).

[27] B. Aubert et al. [BABAR Collaboration], "Measurement of $D^{0}-\bar{D}^{0}$ mixing from a time-dependent amplitude analysis of $D^{0} \rightarrow K^{+} \pi^{-} \pi^{0}$ decays," arXiv:0807.4544 [hep$\mathrm{ex}]$.

[28] D. M. Asner et al. [CLEO Collaboration], "Determination of the $D^{0} \rightarrow K^{+} \pi^{-}$Relative Strong Phase Using Quantum-Correlated Measurements in $e^{+} e^{-} \rightarrow D^{0} \overline{D^{0}}$ at CLEO," Phys. Rev. D 78, 012001 (2008).

[29] E. Barberio et al. [Heavy Flavor Averaging Group], "Averages of b-hadron and $c$-hadron Properties at the End of 2007," arXiv:0808.1297v3 [hep-ex] 14 Mar 2009.

[30] C. S. Mishra, "High Luminosity Operation of the Fermilab Accelerator Complex," In the Proceedings of Particle Accelerator Conference (PAC 03), Portland, Oregon, 12-16 May 2003, pp 1.

[31] CDF II Detector Web Page: http://www-cdf.fnal.gov/events/detector_pictures.html.

[32] E. S. McCrory, "Beam diagnostics for the Fermilab 400-MeV Linac upgrade," FERMILAB-LU-218.

[33] R. C. Webber and G. Apollinari, "Overview and Status Update of the Fermilab HINS Linac R\&D Program," FERMILAB-CONF-09-131-APC.

[34] J. Lackey, F. G. Garcia, M. Popovic and E. Prebys, "Operation and performance of the new Fermilab Booster $H^{-}$injection system," In the Proceedings of Particle Accelerator Conference (PAC 07), Albuquerque, New Mexico, 25-29 Jun 2007, pp 1709. CONFP, C070625, 1709.

[35] R. Zwaska, S. Kopp, W. Pellico and R. Webber, "Synchronization of the Fermilab booster and main injector for multiple batch injection," arXiv:physics/0407007.

[36] C. M. Bhat, "The main injector facility: A new tool for high energy physics at Fermilab," Int. J. Mod. Phys. A 16S1C, 1187 (2001). 
[37] P. T. Lukens [CDF IIb Collaboration], "The CDF IIb Detector: Technical Design Report," CDF-DOC-CDF-PUBLIC-6261.

[38] J. Antos et al. [CDF - Run II Collaboration], "Data processing model for the CDF experiment," IEEE Trans. Nucl. Sci. 53, 2897 (2006).

[39] A. Sill [CDF Collaboration], "CDF Run II silicon tracking projects," Nucl. Instrum. Meth. A 447, 1 (2000).

[40] A. A. Affolder et al. [CDF Collaboration], "CDF central outer tracker," Nucl. Instrum. Meth. A 526, 249 (2004).

[41] B. Ashmanskas et al. [CDF-II Collaboration], "The CDF silicon vertex trigger," Nucl. Instrum. Meth. A 518, 532 (2004).

[42] E. J. Thomson et al., "Online track processor for the CDF upgrade," IEEE Trans. Nucl. Sci. 49, 1063 (2002).

[43] A. Abulencia et al. [CDF Collaboration], "Measurement of the ratio of branching fractions $\mathrm{B}\left(\mathrm{D} 0 \rightarrow K^{+} \pi^{-)} / \mathrm{B}\left(\mathrm{D} 0 \rightarrow K^{-} \pi^{+}\right)\right.$using the CDF II Detector," Phys. Rev. D 74, 031109 (2006).

[44] M. Mattson, P. Karchin, and N. Kulkarni, "Time dependent analysis of $D^{0}-\overline{D^{0}}$ mixing," version 1.4, (private communication).

[45] M. Mattson, P. Karchin, and N. Kulkarni, "Analysis of $D^{0}-\overline{D^{0}}$ mixing in the $K \pi$ channel," version 1.5, (private communication).

[46] C. Amsler et al. [Particle Data Group], "Review of particle physics," "Statistics," Phys. Lett. B 667, 1 (2008).

[47] M. Feindt and U. Kerzel, "The NeuroBayes neural network package," Nucl. Instrum. Meth. A 559, 190 (2006).

[48] T. Sjostrand, S. Mrenna and P. Skands, "A Brief Introduction to PYTHIA 8.1," Comput. Phys. Commun. 178, 852 (2008). 
[49] T. Sjostrand, S. Mrenna and P. Skands, "PYTHIA 6.4 Physics and Manual," JHEP 0605, 026 (2006). 
ABSTRACT

ANALYSIS OF $D^{0}-\overline{D^{0}}$ MIXING IN $D^{0} \rightarrow K \pi$ DECAYS USING THE CDF II DETECTOR

by

\section{NAGESH P. KULKARNI}

2009

Advisor: Dr. Paul E. Karchin

Major: Physics

Degree: Doctor of Philosophy

We measure the ratio of doubly Cabibbo suppressed $\left(D^{0} \rightarrow K^{+} \pi^{-}\right)$decay rate to Cabibbo favored $\left(D^{0} \rightarrow K^{-} \pi^{+}\right)$decay rate as a function of $D^{0}$ decay time. We used an integrated luminosity of $4.0 \mathrm{fb}^{-1}$ of proton anti-proton collisions at $\sqrt{s}=1.96 \mathrm{TeV}$ at the collider detector at Fermilab (CDF II). A signal of $24000 D^{0} \rightarrow K^{+} \pi^{-}$decays was obtained in this experiment. We measure the $D^{0}-\overline{D^{0}}$ mixing parameters $R_{D}, y^{\prime}$, and $x^{\prime 2}$ from the ratio. We find that the data excludes the no-mixing hypothesis with probability equivalent to 5.3 Gaussian standard deviations. This amounts to an observation of $D^{0}-\bar{D}^{0}$ mixing in $D^{0} \rightarrow K \pi$ channel. 


\section{AUTOBIOGRAPHICAL STATEMENT}

Name: NAGESH P. KULKARNI

\section{Education:}

Ph.D. Physics, Wayne State University, Detroit, Michigan,USA, 2009.

M.S. Physics, Wayne State University, Detroit, Michigan,USA, 2006.

\section{Research Experience:}

High energy particle physics, Dept. of Physics and Astronomy, WSU, 2005-2009.

System Expert: Calorimeter Electronics at CDF II, Fermi National Accelerator Lab, (July 2006 to June 2007).

System Expert: Trigger monitoring at CDF II, Fermi National Accelerator Lab, (July 2006 to June 2007).

\section{Professional Experience}

Software Development Process Consultant, General Motors (January 2004 to December 2004).

Software Engineer-Telecommunication Engineering, Nortel Networks (December 1999 to March 2003).

Software Engineer-Computer Graphics, Tangent Software Pvt. Ltd (April 1998 to December 1999). 